\title{
Columbia University in the City of New York
}

\section{LAMONT GEOLOGICAL OBSERVATORY}

PALISADES. NEW YORK

\section{MAGNETIC ANOMALIES CAUSED BY TWO-DIMENSIONAL STRUCTURE: THEIR COMPUTATION BY DIGITAL COMPUTERS AND THEIR INTERPRETATION \\ by}

J. R. Heirtzler, G. Peter, M. Talwani and E. G. Zurflueh

Technical Report No. 6 CU-6-62 Nonr-Geology 



\section{LAMONT GEOLOGICAL OBSERVATORY \\ (Columbia University) \\ Palisades, New York}

MAGNETIC ANOMALIES CAUSED BY TWO-DIMENSIONAL STRUCTURE:

THEIR COMPUTATION BY DIGITAL COMPUTERS

AND

THEIR INTERPRETATION

by

J.R. Heirtzler, G. Peter, M. Talwani and E.G. Zurflueh

Technical Report No. 6

CU-6-62-Nonr - Geology

October 1962 

CONTENTS

Page

CHAPTER I Introduction

by J.R. Heirtzler and E.G. Zurflueh I-I

CHAPTER II The Mathematical Expression for the

Magnetic Anomaly over a Two-Dimensional

Body of Polygonal Cross Section

by M. Talwani and J.R. Heirtzlor

$2-7$

A. Derivation of the Basic Formulas 2-1

B. Induced and Remanent Magnetization and the Total Intensity Anomaly 2-10

CHAPTER III Computed Magnetic Total Intensity Curves over Two-Dimensional Bodies

by G. Peter

A. Description of the Model 3-1

B. Discussion of the Computed Curves 3-2

CHAPTER IV A Method of Interpretation for Anomalios of Total Magnetic Intensity caused by Two-Dimensional Bodies

by E.G. Zurflueh

$4-1$

A. General Remarks

4-1

B. Mathematical Expressions

4-3

C. Description of Method

$4-10$

D. Results of Practical Applications

$4-15$

E. Curves and Graphs

$4-26$

A PPENDIX

A Digital Computer Program for the Computation of Magnetic Anomalies over Two-Dimensional Polygonal Bodies by M. Talwani and J.R. Heirtzler

A. Framing the Problem A-I

B. Flow Diagram

A -4

C. A Simple Example A-10

ACKNOWLEDGEMENTS

$R-1$

REFERENCES

$\mathrm{R}-1$ 
Digitized by the Internet Archive in 2020 with funding from Columbia University Libraries 


\section{Chapter I}

Introduction

J.R. Heirtzler and E.G. Zurflueh

In a sense this is a "housekeeping" report in that it brings together, in a more or loss orderly fashion, for our own use some of the analysis techniques that have been used by various persons at the Iamont Geological Observatory. It is felt that such material may be of interest to individuals initiating an analysis of the geomagnetic field intensity for purposes of determining earth structure.

In the measurements of the geomagnetic field intensity in marine areas by Lamont it has seldom been possible to take measurements over a systematic grid and construct magnetic contour charts. Measurements usually consist of a magnetic profile along the track of a research vessel, these measurements being taken with a towed magnetometer that records the total intensity of the geomagnetic field. Over a period of time our ships and other ships and aircraft have made sufficient measurements in some areas to permit a general description of the magnetic anomalles even though track spacing and heading prohibit contourIng. Frequently such a general description can substantiate or refute geophysical hypotheses and suggest guide-Iines for future investigations.

An attempt to doduce exact geophysical information from a limited magnetic profile is recognized as a highly uncertain undertaking, especially when control of the time 
variations is doubtful. Alternatively, analyzing a high density of profiles on a statistical basis may be of value if no errors in basic physics are committed and the limitations of the techniques are understood.

The structures causing magnetic anomalies can frequently be treated as cylinderical, or two-dimensional, i.e. extending to plus and minus infinity in a direction parallel to a coordinate axis. Two-dimensional structure seems to be particularly common in certain marine areas, see for example (Vacquier, et.al., 1961) and (Heirtzler, et.al., 1962). It is instructive in such cases to determine some of the configuations of magnetic materials that can produce anomalies similar to those observed. An electronic digital computer program to calculate the anomaly that would be caused by an assumed twodimensional structure has been used by this institution for several years. It was originally written as a program for the IBM 650 by M. Landisman of Lamont. It was written again for the IBM 1620, because of the convenience of that computer. It is the formulation of this second program that is given here but, naturally, both programs give the same answers. In order to give some crude feeling of how the various geometrical factors affect the total field anomalies a brief selection of anomalies for simple bodies is given in Chapter III. With the large number of geometrical parameters that are important in this calculation it is clearly impossible to give a complete handbook of anomalies with one parameter varying at a time. The 
relative importance of the parameters is discussed in Chapters III and IV.

In Chapter IV a close examination is made of the calculated anomalies for those bodies with vertical sides. A working set of rules is presented to determine the configuration of such bodies from observed profiles. In that Chapter it is also shown how far mathematical theory can be carried without having to make very specialized assumptions about body configuration.

In connection with the presentation of interpretational techniques in this paper, a brief listing of some of the pertinent literature is given here with short comments on the contents of each publication.

Several authors give sets of model anomalies, usually accompanied by rules for depth determination and formulas for the particular model used. In this way Haalck (1927), Gulatee (1938) and Heiland (1946) compute model anomalies of the horizontal and vertical components for different bodies. Nettleton (1942) determines the vertical intensity anomaly for various geometric configurations. Henderson and Zietz (1948) treat the effect of point and line sources with respect to total intensity measurements. In 1958 the same authors published a paper on the use of magnetic doublets in the interpretation of total intensity anomalies. Vacquier, et.al. (1951) interpret total intensity maps with the help of three-dimensional (prismatic) models and computed second vertical derivatives. Zietz and Henderson (1954) use three-dimensional double layer model fields 
for the analysis of total intensity data. Baranov (1955) standardizes his model calculations by reducing measurements for different inclinations to vertical field conditions. Smellie (1956) gives total intensity depth factors for point and Iine configurations of poles and dipoles. From general mathematical considerations Smith (1959 and 1961) derives inoqualities which can be used to estimate depth and intensity of magnetization for arbitary bodies.

Before the advent of the airborne magnetometer the horizontal or vertical components were measured almost exclusively and the model anomalies were treated accordingly. Since about 1946 total intensity measurements are used in general and the newer literature usually refers to total intensity anomalies. According to potential theory it is possible to calculate any component from measurements of any other component if certain conditions are fulfilled (for example see symmetry relations on page 2-9). A group of papers deal with the conversion of one component into another and with the related problems of computing derivatives and upward and downward continuation.

Skeels (1947) was the first to explicitly point out the general possibility of the above mentioned conversions by surface integration. Vestine (1941) and Vestine and Davids (1945) also point out the importance of integral methods in magnetic interpretation. Hughes and Pondrom (1947) describe the calculation of the vertical intensity out of total intensity 
data. Skeels and Watson (1949) treat the problem of conversions in a more general way. Henderson and Zletz (1949a) give a method for the computation of second vertical derivatives and In another peper (1949b) they analyze the upward continuation of the field. Peters (1949) treats the problems of upward and downward continuation and the calculation of derivatives. He also derives methods for the direct determination of basement structure. Henderson (1960) relates the different operations of conversion to one basic formula and uses expressions suitable for use on electronic computers.

Another group of papers is concerned with the inverse problem of the calculation of magnetic anomailes caused by bodies of any given shape. Gassmann (1951) describes a graphical method of effecting the triple integration necessary for the solution of this problem. Baranov (1953) works out another graphical method with a different sequence of integrations. Henderson and Zietz (1957) simplify the computation of a total intensity anomaly by projecting a map of a given body in the direction of the earth's field and using a modified Gassmann process for the integration. Talwani and Ewing (1960) calculate the anomaly by dividing the body into parallel and polygonal laminae, similar to the treatment in this report. Their method is put in a form which can be used for digital computers. Vajk (1951) and Nettleton (1954) analyze the first step in magnetic anomaly interpretation, namely the separation 
of anomalies from an assumed regional field. A summary of the

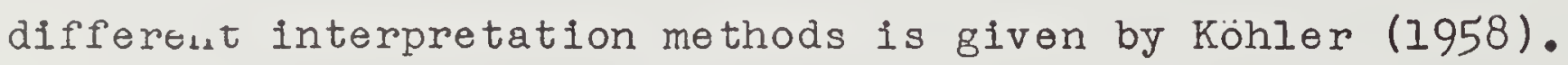
His work also contains a rather complete list of references which includes papers from the large Russian literature on this subject. Lastly, there are extensive, company classified techniques used by the geophysical exploration companies. 
The Mathematical Expression for the Magnetic Anomaly over a Two-Dimensional Body of Polygonal Cross Section

M. Talwani and J.R. Hөirtzler

A. Derivation of the Basic Formulas

It is characteristic of geophysics texts to derive the expressions for the horizontal and vertical magnetic anomalies by analogy with the expressions for gravitational anomalies. In the present report the expressions for the horizontal, vertical, and total field anomalies are derived from basic magnetostatic theory.

Consider the volume element $\Delta x, \Delta y, \Delta z$ located in an external magnetic field (Fig. 2-I).

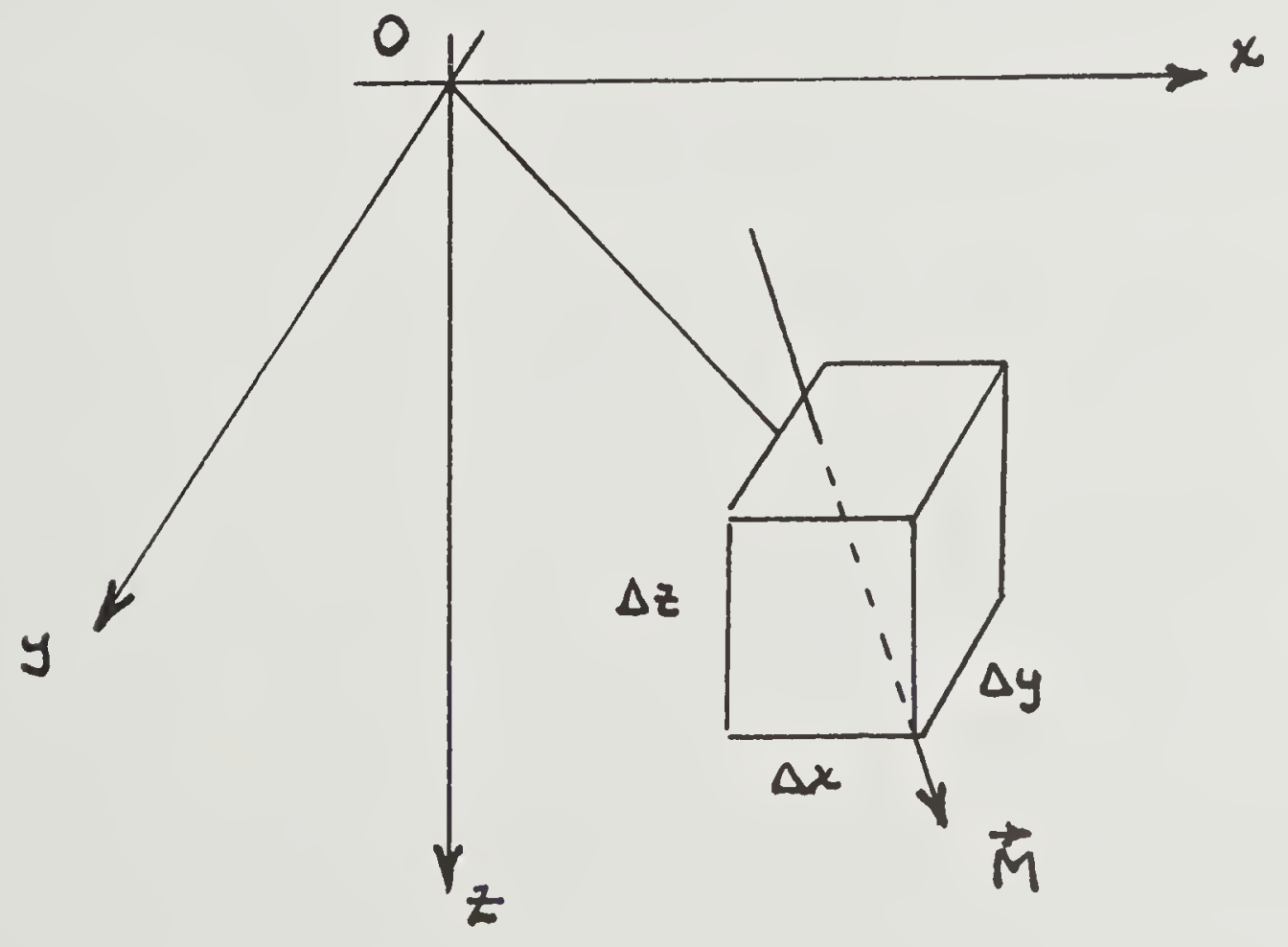

Fig. 2-1 
$2-2$

We assume that the volume element has a uniform magnetization $\vec{M}$. Let $\vec{\mu}$ be the magnetic moment of the element. Then

$$
\vec{\mu}=\vec{M} \Delta x \Delta y \Delta z
$$

The magnetic potential, at the origin, due to the volume element is

$$
P=\frac{\vec{\mu} \cdot \vec{F}}{r^{3}}=\frac{M_{x} x+M_{y} y+M_{z} z}{\left(x^{2}+y^{2}+z^{2}\right)^{1 / 2}} \Delta x \Delta y \Delta z
$$

where

$$
\vec{r}=\vec{i} x+\vec{j} y+\vec{k} z
$$

At the origin the potential of a rod of cross section $\Delta x \Delta z$ and infinite in the $y$-direction is

$$
\begin{aligned}
P & =\Delta x \Delta z \int_{-\infty}^{\infty} \frac{M_{x} x+M_{y} y+M_{z} z}{\left(x^{2}+y^{2}+z^{2}\right)^{3 / 2}} d y \\
& =2 \Delta x \Delta z\left\{\frac{M_{x} x+M_{z} z}{x^{2}+z^{2}}\right\} .
\end{aligned}
$$

The vertical magnetic field strength, $V$, is

$$
V=-\frac{\partial P}{\partial z}=2 \Delta x \Delta z\left\{\frac{z x z M_{x}-M_{z}\left(x^{3}-z^{2}\right)}{\left(x^{2}+z^{2}\right)^{2}}\right\}
$$


and the horizontal field strength, $H$, measured in the $x$ direction is

$$
H=-\frac{\partial P}{\partial x}=2 \Delta x \Delta z\left\{\frac{M_{x}\left(x^{2}-z^{2}\right)+2 x z M_{z}}{x^{2}+z^{2}}\right\}
$$

The horizontal field strength measured in the $y$-direction is zero since $y$ is not contained in the expression for the potential. If $\vec{M}$ is the induced magnetization this means, physically, that wo have not included demagnetization offects. The above represent the magnetic anomalies caused by the rod, that is the regional field strength is not included.

Consider the lamina shown in Fig. 2-2, infinito in the $y$-direction and infinite in the positive $x$-direction.

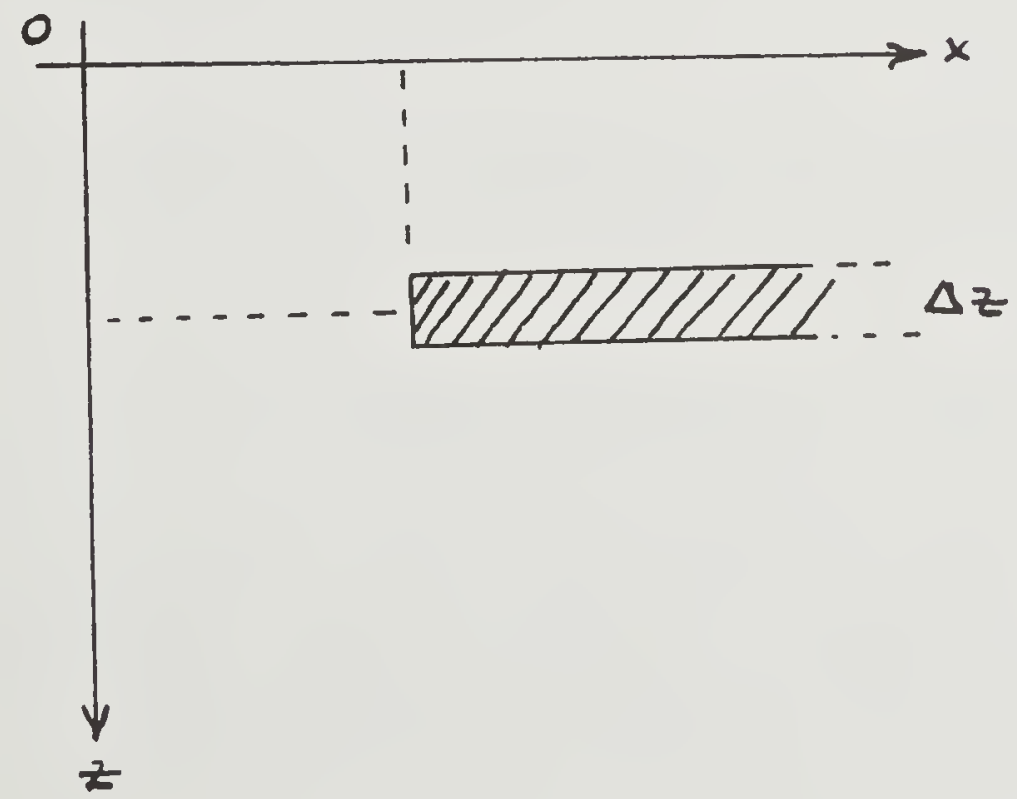

Fig. 2-2 
By the use of eqs (2-1) and (2-2) one gets

$$
\begin{aligned}
V & =2 \Delta z \int_{x}^{\infty} \frac{H_{x} 2 x z-M_{z}\left(x^{2}-z^{2}\right)}{\left(x^{2}+z^{2}\right)^{2}} d x \\
& =2 \Delta z\left\{\frac{M_{x} z-M_{z} x}{x^{2}+z^{2}}\right\}
\end{aligned}
$$

$$
\begin{aligned}
H & =2 \Delta z \int_{y}^{\infty} \frac{M_{x}\left(x^{2}-z^{2}\right)+M_{z} 2 x z}{\left(x^{2}+z^{2}\right)^{2}} d x \\
& =2 \Delta z\left\{\frac{M_{x} x+M_{z} z}{x^{2}+z^{2}}\right\}
\end{aligned}
$$

The variables $x$ and $z$ in $(2-3)$ and $(2-4)$ represent the coordinates of the ond of the lamina. If we had taken the lamina running in the opposite direction, as indicated in Fig. 2-3 
$2-5$

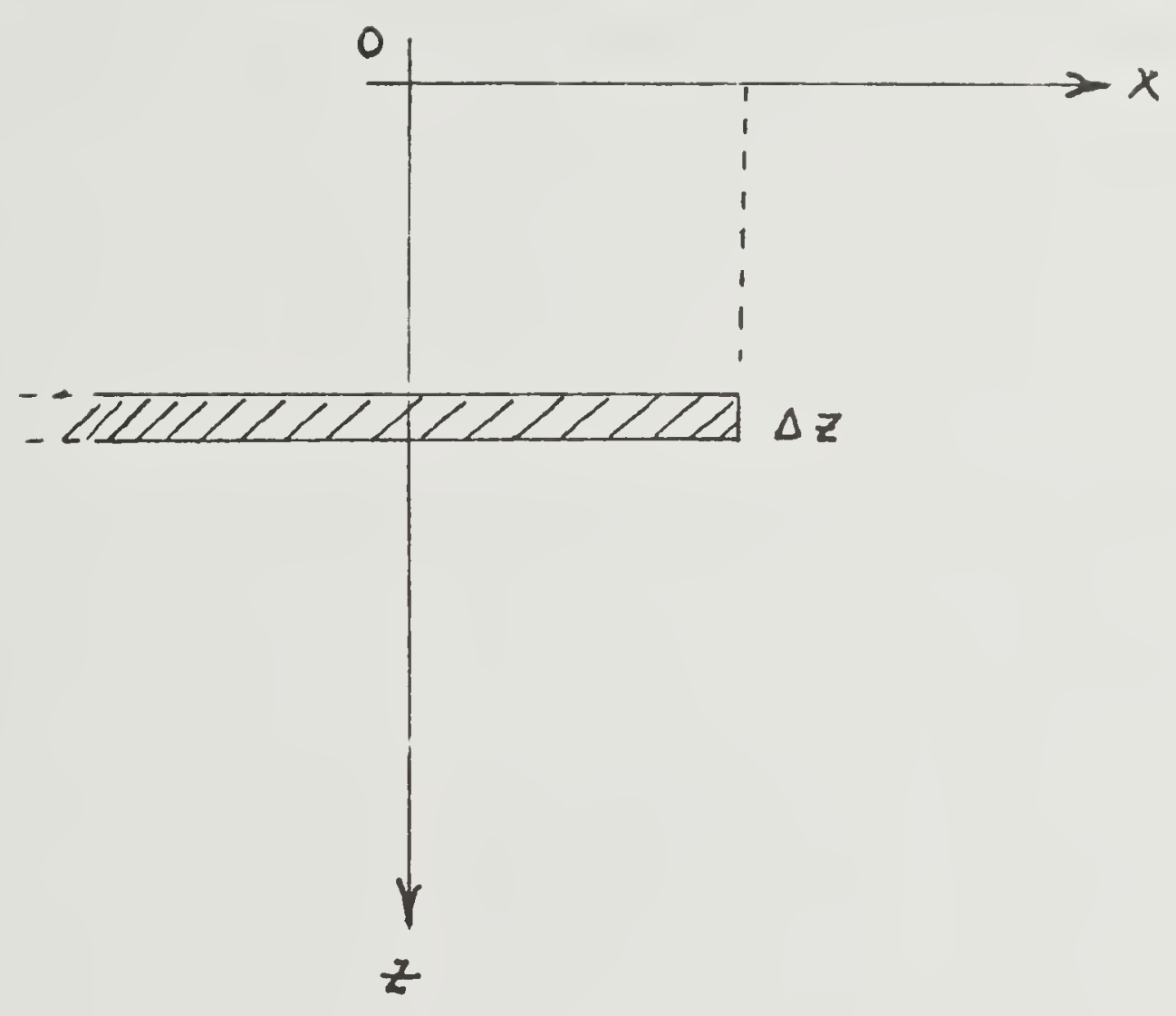

Fig. 2-3

wo would have obtained

$$
\begin{aligned}
& V=2 \Delta z\left\{\frac{-M_{x} z+M_{z} x}{x^{2}+z^{2}}\right\} \\
& H=2 \Delta z\left\{\frac{-M_{x} x-M_{z} z}{x^{2}+z^{2}}\right\}
\end{aligned}
$$


Notice that $\mathrm{V}$ and $\mathrm{H}$ bear the opposite signs to eqs (2-3) and (2-4). Next consider the prism shown in Fig. 2-4, infinite in the $\mathrm{y}$ direction.

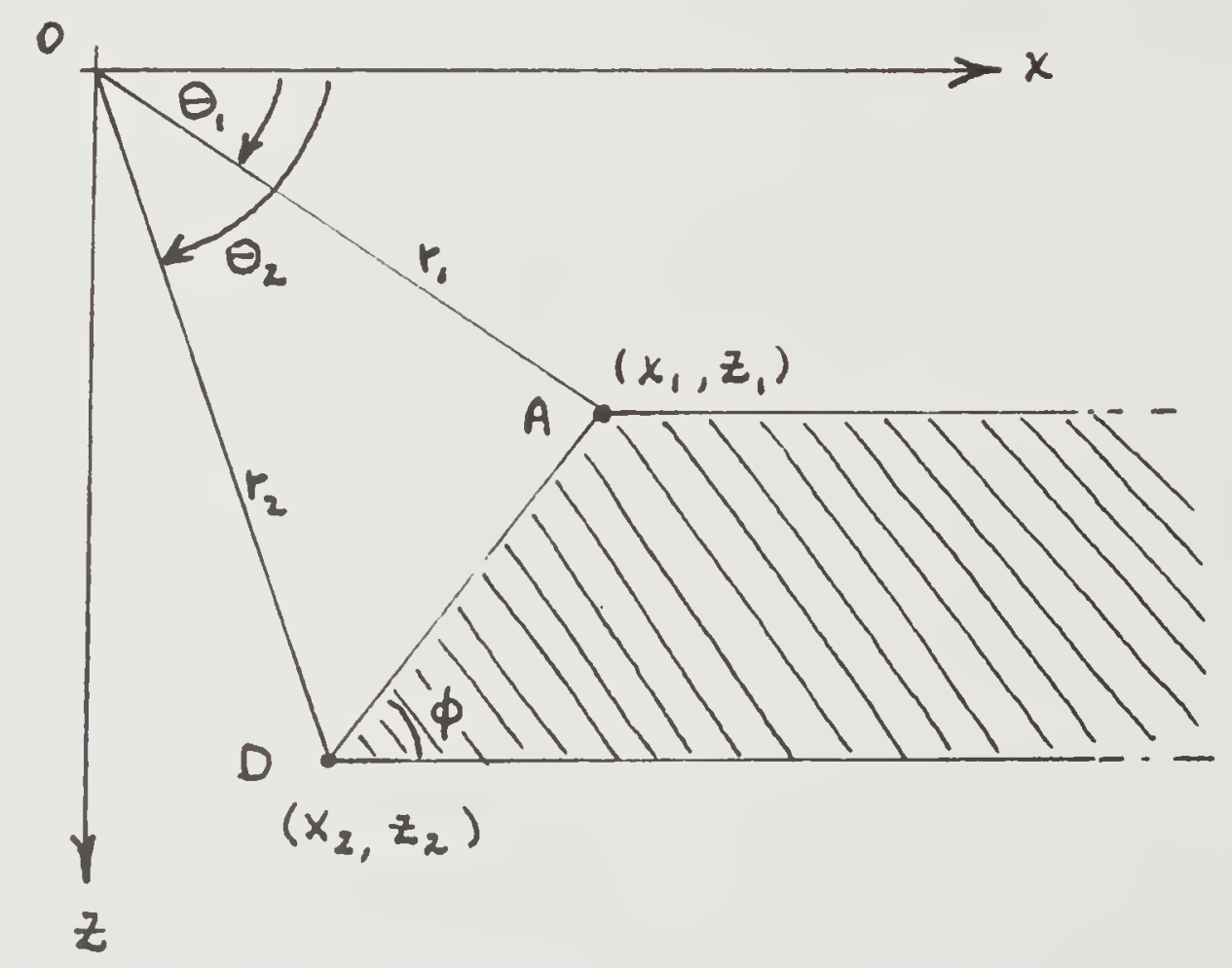

Fig. $2-4$

The expressions for $\mathrm{V}$ and $\mathrm{H}$ at the origin can be obtained by 
the integration with respect to $z$ in eqs $(2-3)$ and $(2-4)$ :

$$
\begin{aligned}
& V=2 \int_{z_{1}}^{z_{2}}\left\{\frac{M_{x} z-M_{z} x}{x^{2}+z^{2}}\right\} d z \\
& H=2 \int_{z_{1}}^{z_{2}}\left\{\frac{M_{x} x+M_{z} z}{x^{2}+z^{2}}\right\} d z
\end{aligned}
$$

The integration is carried out from the small to the larger $\theta$. In eqs $(2-3)$ and $(2-4)$ the variables $x$ and $z$ represented the coordinates of the end of the lamina. For the prism $x$ and $z$ are related by the equation for the line $A D$ :

$$
x=\left(x_{1}+z_{1} \cot \phi\right)-(\cot \phi) z_{1}
$$

If eqs (2-5) and (2-6) are integrated and the variables $r$ and used instead of $x$ and $z$ one obtains: 


$$
\begin{aligned}
V & =2 \sin \phi\left[\pi_{k}\left\{\left(\theta_{2}-\theta_{1}\right) \cos \phi+\sin \phi \log \left(r_{2} / r_{1}\right)\right\}\right. \\
& \left.-M_{z}\left\{\left(\theta_{1}-\theta_{1}\right) \sin \phi-\cos \phi \log \left(r_{2} / r_{1}\right)\right\}\right]
\end{aligned}
$$

$$
\begin{aligned}
H & =z \sin \phi\left[M_{x}\left\{\left(\theta_{2}-\theta_{1}\right) \sin \phi-\cos \phi \log \left(r_{2} / r_{1}\right)\right\}\right. \\
& \left.+M_{z}\left\{\left(\theta_{2}-\theta_{1}\right) \cos \phi+\sin \phi \log \left(r_{2} / r_{1}\right)\right\}\right] .
\end{aligned}
$$

Had it been assumed that the prism was orlented as in Fig. 2-5 the above would have been preceded by a minus sign.

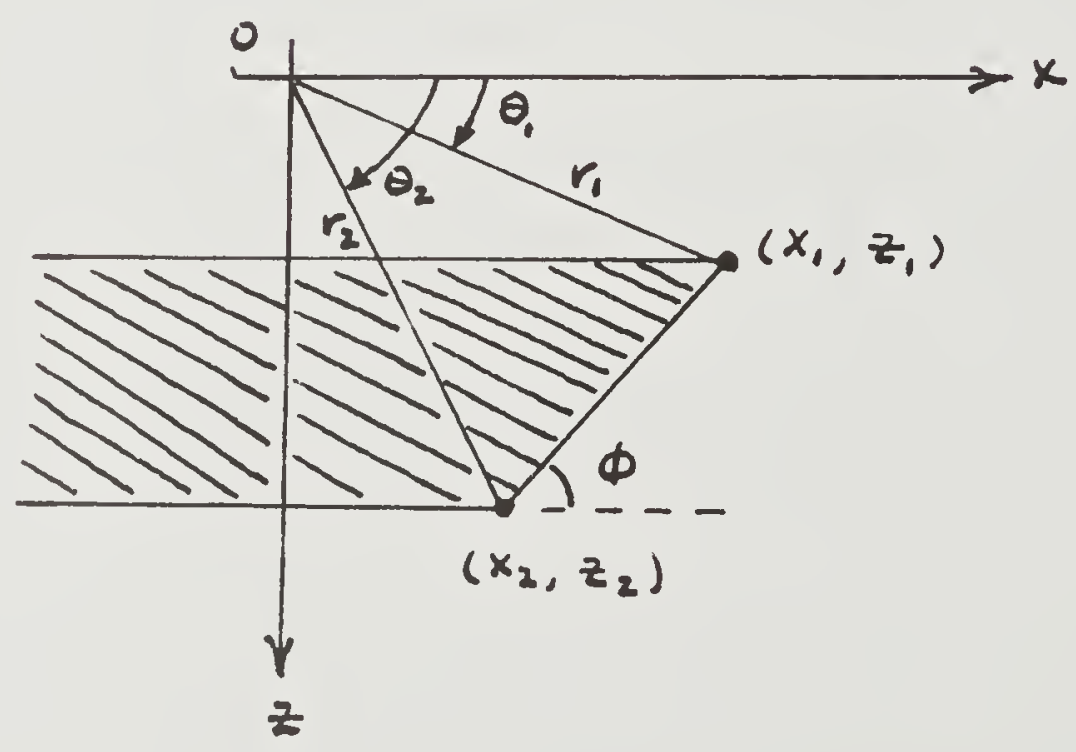

Fig. 2-5 
In Figs. $(2-4)$ and $(2-5)$ the prism extended infinitely in one of the $x$ directions. It is clear that $H$ and $V$ for a body of finite $x$ dimensions can be treated by assuming that it consists of two bodies (ref. Fig. 2-6). One must be careful with directions of angular measurements and with signs in working with these equations.

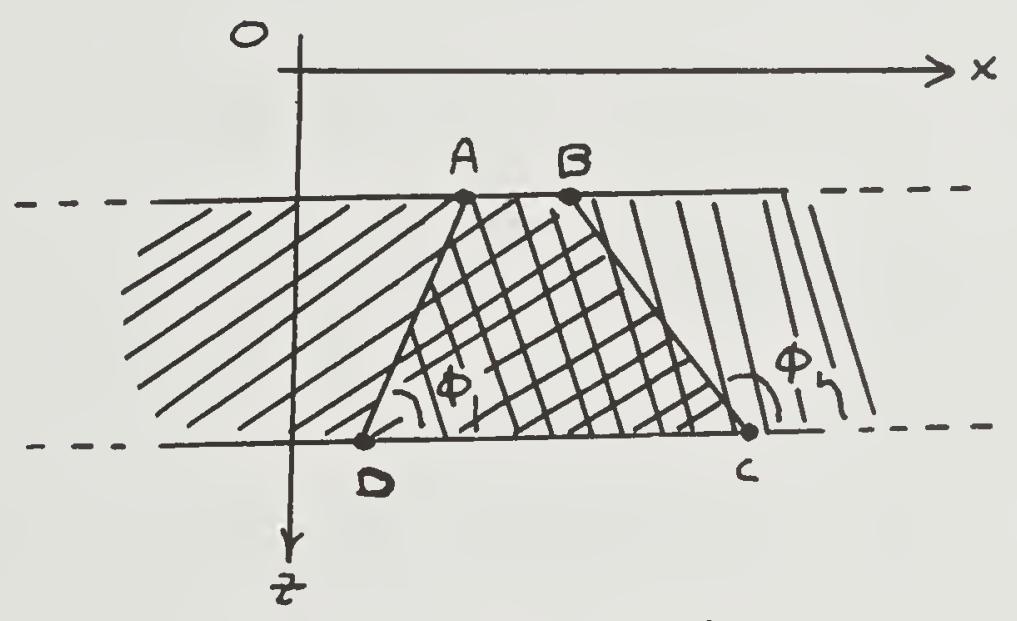

Fig. 2-6

The procedure indicated above can be extended to any number of prismatic or polygonal bodies each with many faces and each with its own magnetization. This derivation does not take into account nonuniform magnetization such as would exist near the corners of the bodies. In fact the actual geological bodies probably do not have distinct comers and nonuniform magnetization is believed to be unimportant.

It is interesting to note the symmetrical relationships of the factors in eqs $(2-7)$ and $(2-8)$. That symmetry shows that vertical magnetization effects the horizontal anomaly to the same extent that the transverse magnetization effects the vertical 
anomaly and that the transverse magnetization effects the horizontal anomaly to the same extent that the vertical magnetization offects the vertical anomaly.

B. Induced and Remanont Magnetization

and the Total Intensity Anomaly

If one wishes to utilize eqs $(2-7)$ and (2-8) for the calculation of the $\mathrm{V}$ and $\mathrm{H}$ anomalies due to induced magnetization only he would write

$$
\overrightarrow{\mathrm{M}}=\mathrm{k} \overrightarrow{\mathrm{F}}
$$

where $\mathrm{k}=$ magnetic suscoptibility

$$
\vec{F} \text { = undisturbed regional total intensity vector }
$$

and as illustrated in Fig. 2-7

$$
M_{X}=k F_{X}=k F \cos I \sin s
$$$$
M_{z}=k F_{z}=k F \sin I
$$

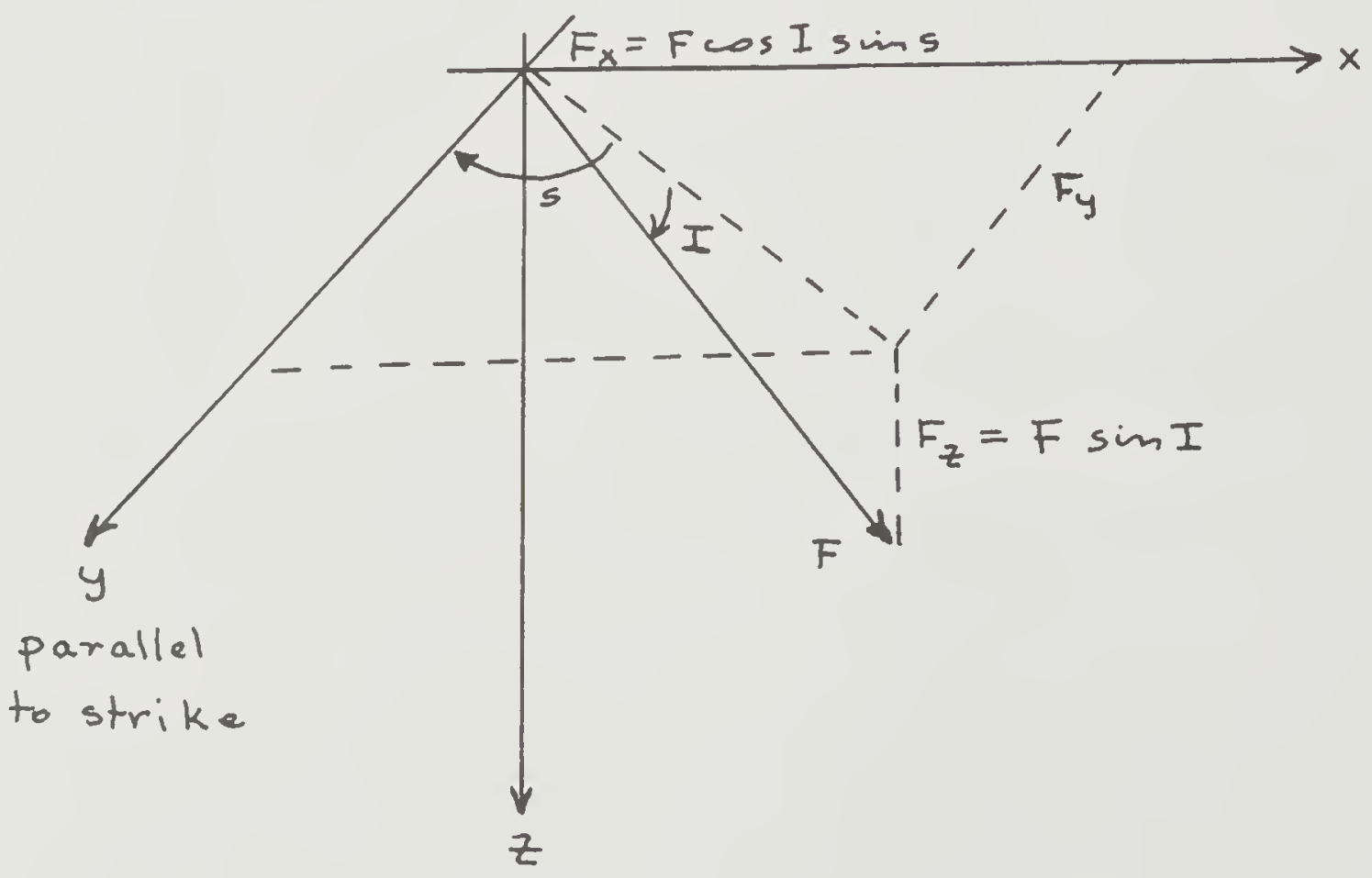

Fig. 2-7 
$I$ = inclination, positive if $F$ is below horizontal (northern hemisphere)

$s=$ angle of strike, measured from horizontal projection of $F$ and either positive or negative $y$ axis. s must always be less than $180^{\circ}$ (and thus sin $s$ positive) since we will specify that the coordinate axes are oriented to make $\mathrm{F}_{\mathrm{x}}$ positive. This orientation of the axes must be borne in mind for the correct interpretation of results.

If one wishes to utilize eqs $(2-7)$ and $(2-8)$ for the calculation of the $\mathrm{V}$ and $\mathrm{H}$ anomalies due to $a$ body with remanent magnetization, and not to include induced anomalies, he would write

$$
\begin{aligned}
& M_{x}=M_{r \ominus m} \cos a \sin b \\
& M_{z}=M_{r e m} \sin a
\end{aligned}
$$

where

$a=$ inclination of remanent magnetization vector below horizontal

$\mathrm{b}=$ strike angle of remanent magnetization vector measured between horizontal projection of $\vec{M}_{\text {rem }}$ and positive or negative $y$ axis, less than $180^{\circ}$. To apply the equations as written the axos must be oriented to make $M_{X}$ positive.

If It is desired to use eqs $(2-7)$ and (2-8) to get the $\mathrm{V}$ and $\mathrm{H}$ anomalies due to the total magnetization (induced and 
remanent) one should write

$$
\begin{aligned}
& M_{x}=M_{t o t} \cos \alpha \sin \beta \\
& M_{y}=M_{\text {tot }} \sin \alpha
\end{aligned}
$$

where

$$
\begin{aligned}
\alpha= & \text { inclination of total magnetization vector below hori- } \\
& \text { zontal } \\
\beta= & \text { strike angle of total magnetization vector measured } \\
& \text { between horizontal projection of } \vec{M}_{\text {tot }} \text { and positive or } \\
& \text { negative } \mathrm{J} \text { axis, less than } 180^{\circ} \text { To apply the equations } \\
& \text { as written, the axes must be oriented to make } \mathrm{M}_{\mathrm{X}} \text { positive. } \\
& \text { Regardless of the assumptions used to determine } \mathrm{V} \text { and }
\end{aligned}
$$

$\mathrm{H}$, the anomaly in total intensity is obtained as follows:

Let $T$ be the anomaly in total intensity, then

$$
(F+T)^{2}=\left(F_{X}+H\right)^{2}+\left(F_{y}\right)^{2}+\left(F_{z}+V\right)^{2}
$$

Since there is no anomaly in the $y$ direction ( $r e f . p \cdot 2-3$ ). Squaring, dropping terms that contain the square of $\mathrm{V}$ or $\mathrm{H}$ and remembering that

$$
F^{2}=F_{x}^{2}+F_{y}^{2}+F_{z}^{2}
$$

get

$$
T=H\left(\frac{F_{x}}{F}\right)+V\left(\frac{F_{z}}{F}\right) .
$$

Rofering to Fig. 2-7

$$
T=H \cos I \sin s+V \sin I .
$$


An electronic digital computer program to calculato $V, H$, and $T$ is given in the Appendix.

Eq (2-9) illustrates that, if the anomalies are small relative to $F$, then the anomaly in total intensity is the component of $\mathrm{V}$ and $\mathrm{H}$ in the Direction of F. See Fig. 2-8.

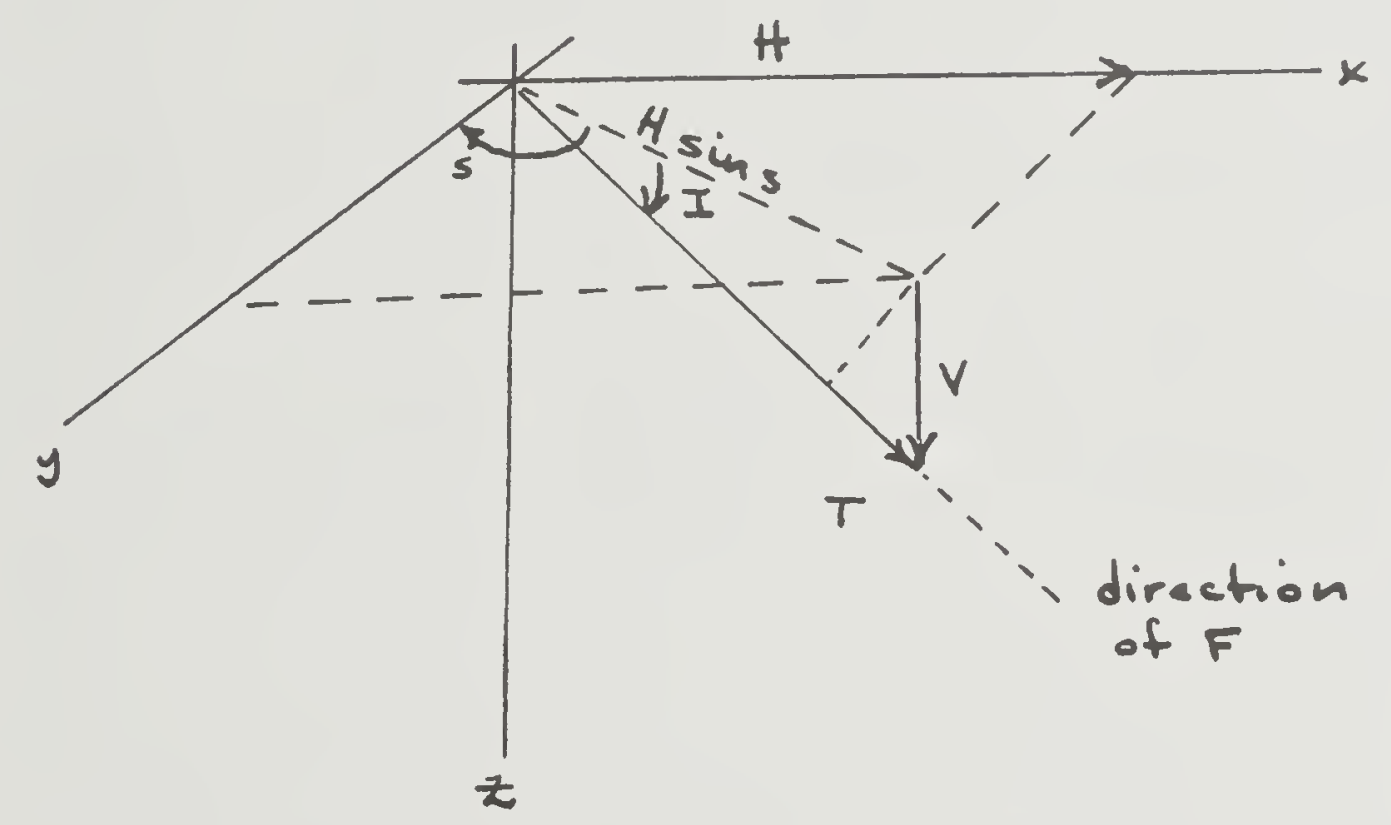

Fig. 2-8

The magnitude of $T$ is composed of the projection of $\mathrm{H}$ ( $H \cos I \sin s)$ and of $V(V \sin I)$ in the direction of $F$. This geometrical picture is invalid if the anomaly is largo (wo dropped squared terms in the derivation of oq 2-9) and a more adequate vector diagram must be used. 
Computed Magnetic Total Intensity Curves over Two-Dimensional Bodies

\section{G. Peter}

\section{A. Description of the Model}

With the aid of the computer program (see Appendix) a few families of magnetic total intensity curves have been computed in order to show the changes in these curves, when the ambient magnetic field directions and the geometric properties of the model are varied. The different sets of curves have been obtained by varying one parameter at a time. Grouping the curves in this manner enables one to make a qualitative analysis of them. The curves are discussed in section $B$.
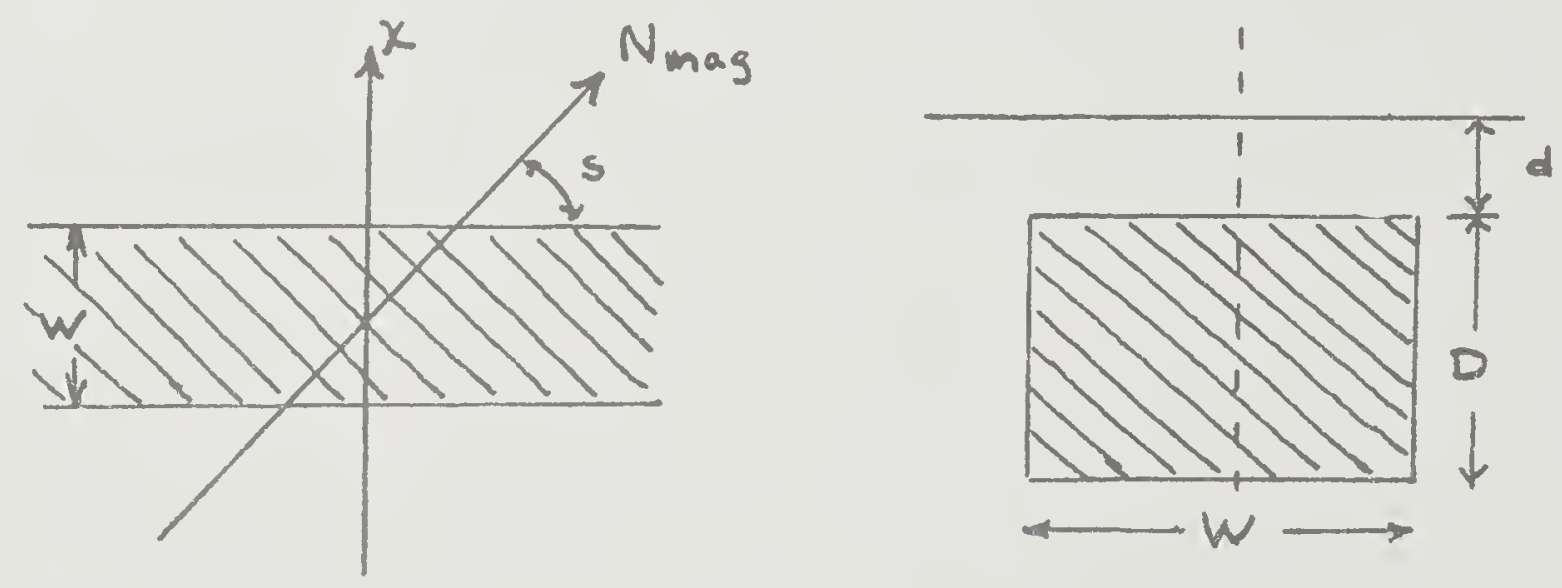

F1g. 3-1: Ground PIan and vertical. section of assumed two-dimensional model
$x=1 \ln \theta$ of measurement
$\mathrm{d}=$ depth of burial
$s=$ strike
$D=$ vertical extent
$W=W i d t h$

The model used in these calculations is a two-dimensiona] rectangular body, which has infinite length in a horizontal 
direction (see fig. 3-1). It has vertical sides, except for the group 6 curves, where the sides have \pm 30 and \pm 60 degree angles from the vertical. For all calculations the model has uniform isotropic induced magnetization of $0.001 \mathrm{emu}$, it is in the northern hemisphere where the fleld strength is 50000 gammas. The projection of the magnetic north on the profiles points toward the right.

The total intensity values are computed for a line of measurement (profile), which is horizontal and at right angle to the axis of the body.

The various curves are grouped as follows:

1. Variable inclination

2. Variable strike

3. Variable depth

4. Variable width

5. Variable vertical extent

6. Slanted bodies
I (figure 3-2)

s (figures $3-3$ to $3-7$ )

d (figures $3-8$ to $3-12$ )

W (figures 3-13 to 3-15)

D (figures 3-16 to 3-18)

(figures $3-19$ to $3-22$ )

These curves are only a selection for a few varied parameters. Additional information can be found in the papers of Haalck (1927), Gulateo (1938) and Helland (1946).

\section{B. Discussion of the Computed Curves}

The following is a short discussion of the general characteristics of the computed curves and of the effect of the various magnetic and model parameters on them. Some elementary characteristics are also mentioned. Conclusions are derived mathematically in Chapters II and IV. 
1. Variable inclination I. (Figure 3-2).

The major features of the magnetic anomaly picture due to induced magnetization in the northern hemisphere consist of a maximum on the south, and a minimum on the north side of a structure. For a symmetrical model, if the strike $s=90^{\circ}$, (the strike $s$ is defined as the angle between the geological strike of the two-dimensional body and the magnetic north) in the case of $I=45^{\circ}$, the size of the maximum and the northerm minimum are equal in amplitude, and the maximum and minimum are symmetrical about the center of the model. For smaller inclinations the northern minimum is the dominating feature. Figure 3-2 also shows that there is a symmetry about $I=45^{\circ}$; in other words for equal inclination differences from $I=45^{\circ}$ the amplitudes of the maximum and minimum are equal, and their positions with the respect of the center of the body are equidistant.

The above statements applied to a symmetrical model are true for the negative inclinations (southern hemisphero) except that the north and south sides of the picture are interchanged.

The above mentioned symmetry around $I=45^{\circ}$ exists because of the symmetrical model used in the calculation, and is due to the change in the relative importance of the horizontal and vertical components of the magnetic field at $I=45^{\circ}$.

2. Variable strike $s$ (Figures $3-3$ to $3-7$ ).

Figures 3-3 to 3-7 show the effect of the strike with various inclinations. It can be seen that with decreasing strike 
angle the maximum of the total intensity curve becomes wider, it moves toward the center of the body, and its minimum toward the north decreases. In the $\mathrm{s}=0^{\circ}$ case there is no minimum (only a maximum with all the inclinations) and the maximu is symmetrical about the center of the body. Because of the type of the model, at $I=0^{\circ}$ and $s=0^{\circ}$ there will be no anomaly observed. The type of the model and the increasing importance of the horizontal component of the magnetic field toward low inclinations requires that the strikes have a greater effect on the low inclination curves.

3. Variable depth

4. Variable width

5. Variable vertical extent d (Figures 3-8 to 3-12)

W (Figures 3-13 to 3-15)

D (Figures 3-16 to 3-18)

These three parameters of the model were individually changed, keeping all the other parameters constant, in order to see how the shape of the total intensity curve respond to these changes at various inclinations.

The variable depth curves show wider and smaller anomalies with increasing depth. The variable width curves cannot be analyzed alone, because the shape of the curve depends on the $w / d$ ratio. It can be deduced from these curves that when the $\mathrm{w} / \mathrm{d} \leqslant$ 1, the horizontal extension (A) of the anomaly curve (the horizontal extension at $50 \%$ maximum value, or $50 \%$ minimum value at low inclinations) is quite insensitive to the width of the body. When $W / d \geqslant 5$, the change of A almost entirely depends on the width, and A approximately equals W. 
The flattening of the maximum peak, or at low incl1nation of the minimum peak, is due to the effect of the lower edge of the model; that is, the effect of the vertical extent of the model D. On the curves near $I=45^{\circ}$ there is a shallowing between the maximum and minimum due to this effect (see Fig. 3-17) which can clearly be seen at smaller values of $D$. This flattening effect is evident when $D \leqslant W$. When the width is much greater than the vertical extent, the maximums or minimums of the anomaly curve will appear at the edges of the body, while over the center the curve will be shallow, and nearly horizontal. This effect can be seen also on the variable width curves at $\mathbb{W}=$ $15 \mathrm{~km}$, when the width of the model is equal to its vertical extent. It is evident that there is an altitude of observation above which the effect of the lower edge cannot be observed.

\section{Slanted bodies (Figures 3-19 to 3-22)}

The present calculations are over four different models. The tops are horizontal and symmetrical about the center of the coordinate system, the sides are $30^{\circ}$ and $60^{\circ}$ from the vertical toward the north, and on the other two models, toward the south. The lower edges are horizontal also, and they are at $15 \mathrm{~km}$ depth. The magnetic total intensity has been calculated for three inclinations over these models, in order to show the change on these curves due to the asymmetry of the models.

The general picture of the magnetic total intensity over these asymmetrical bodies is the same as for symmetrical bodies: there is a maximum to the south, and a minimum to the north of the 
body (northern hemisphere), but now this maximum or minimum does not depend entirely on the inclination, but depends upon the shape of the body also. The various symmetries in the total intensity picture, which were mentioned at the group I calculations, are only valid for symmetrical models. The total intensity picture is different at all inclinations for an asymetrical body. Because the mass is more distributed, the amplitudes are smaller; over the slopes of the model the anomaly becomes wider, and over the sharp tapering edge near the observation line (surface) the anomaly is sharp and pronounced. These effects can be seen by comparing the two oppositely slanted models: Fig. 3-19, Fig. 3-21, and Fig. 3-20, Fig. 3-22. All four figures show that the shape of the upper part of the model has a dominating effect on the anomaly curve, while the deep extension of the model only causes the widening of the maximums or minimums over the sloping side.

Except for the symetry relations, the same conclusions given in the previous sections can be drawn for these slanted bodies at various strike angles $s$, and the $d$, $W$, and $D$ changes would also have similar effect as they had on the symmetrical models. 


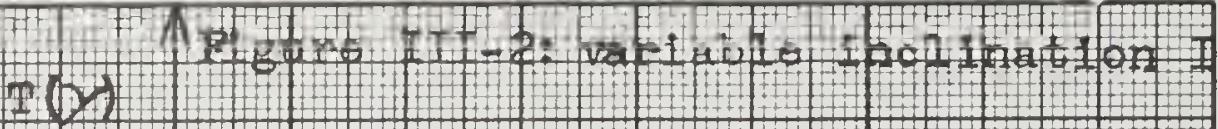

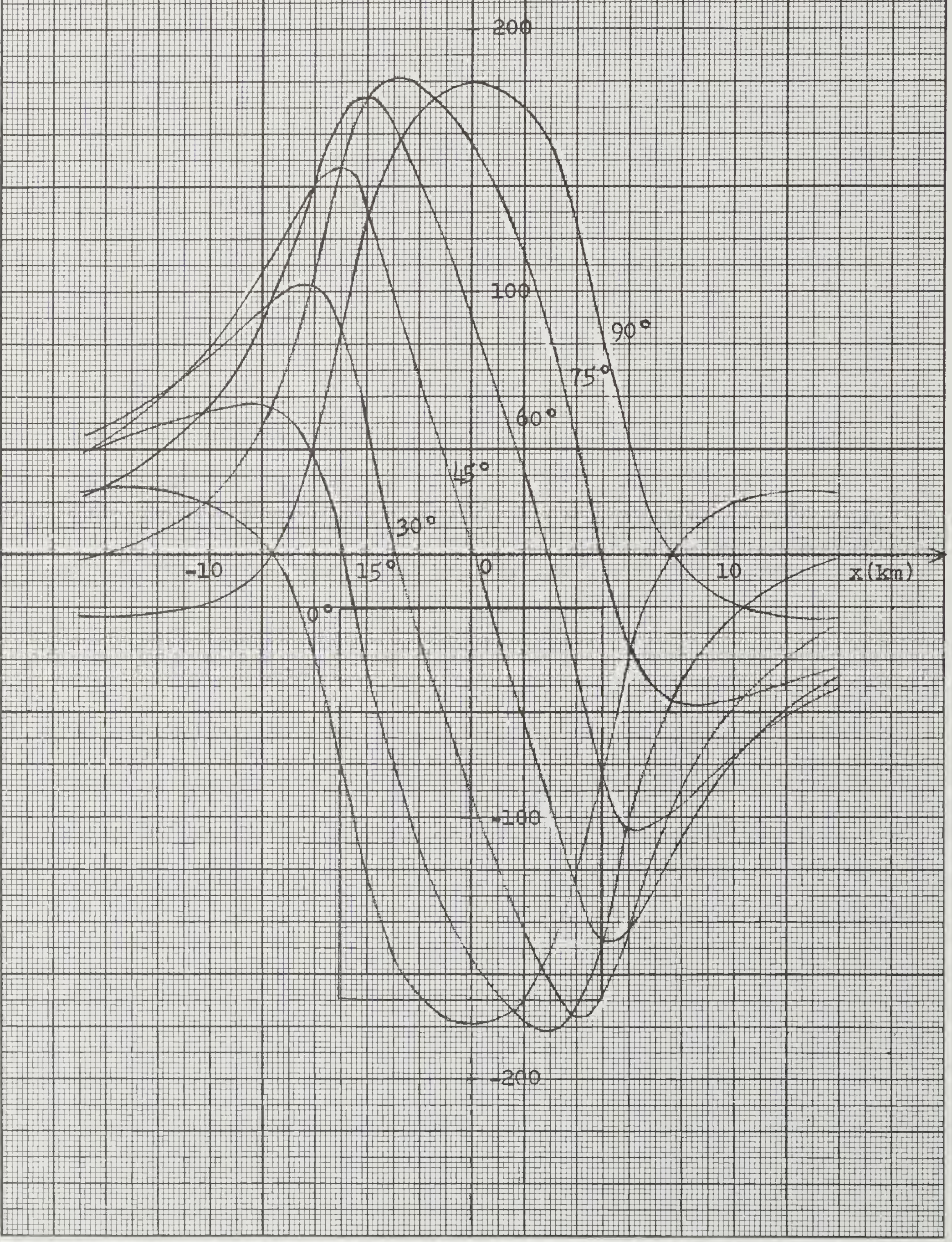




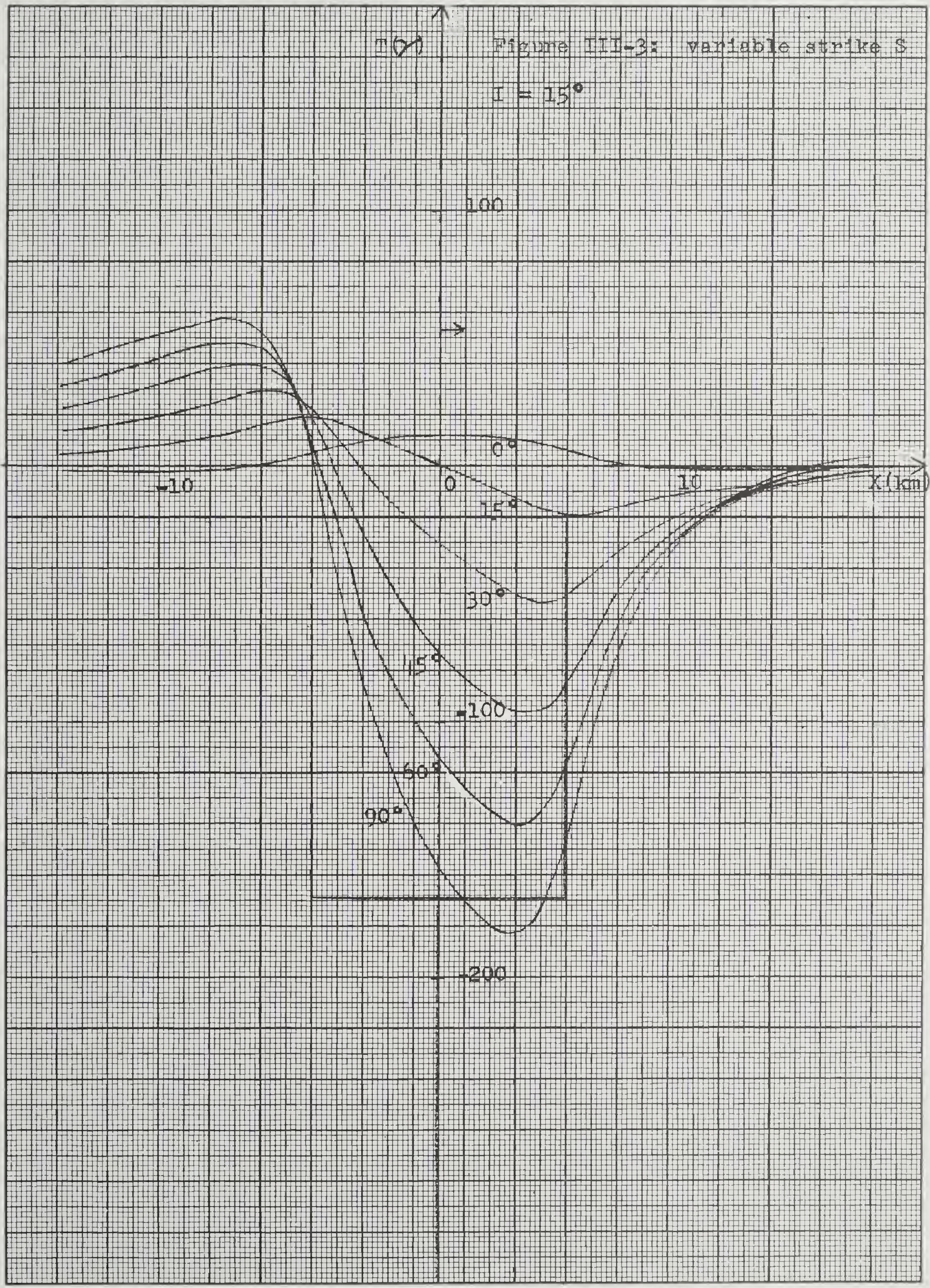




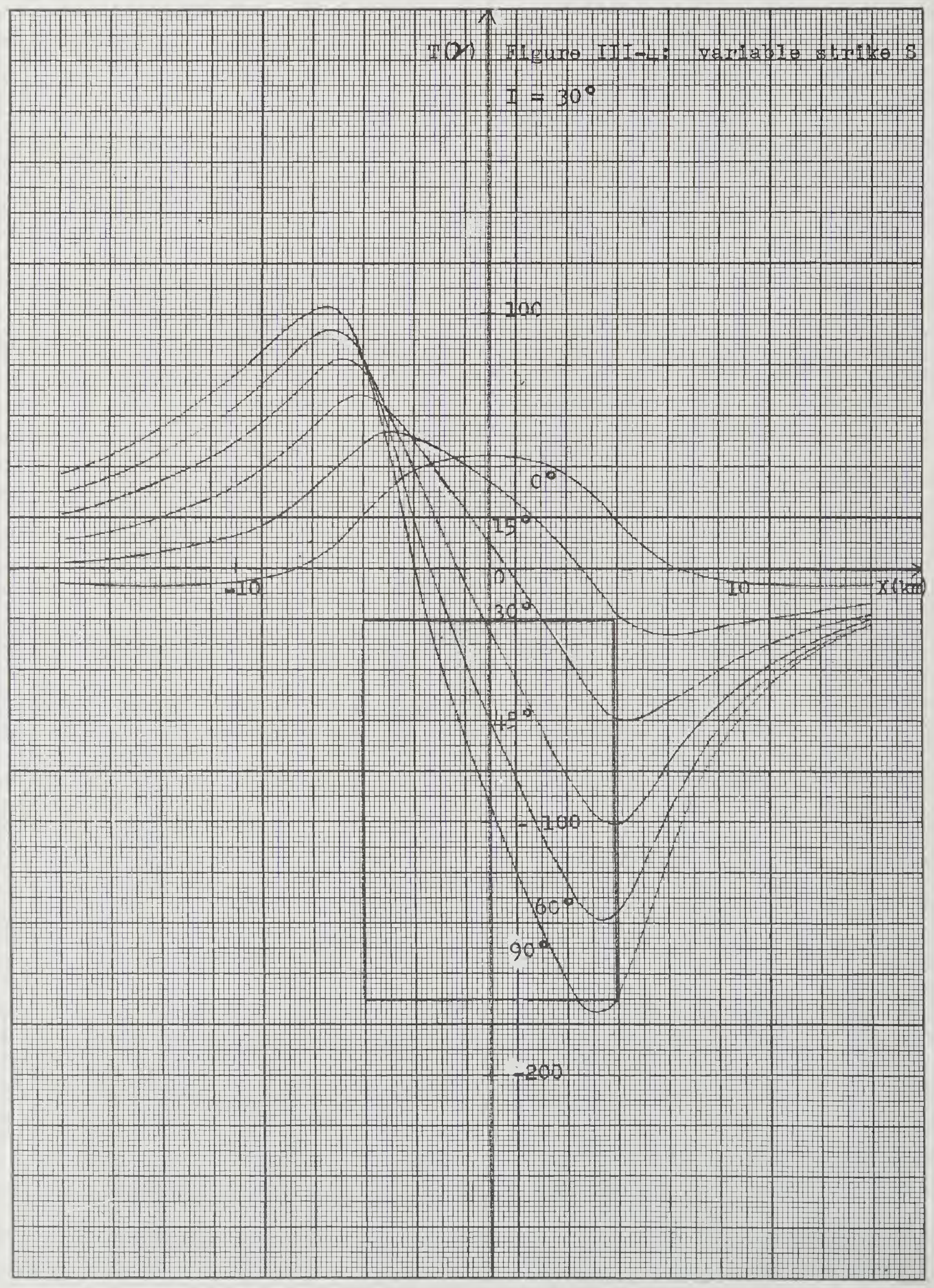




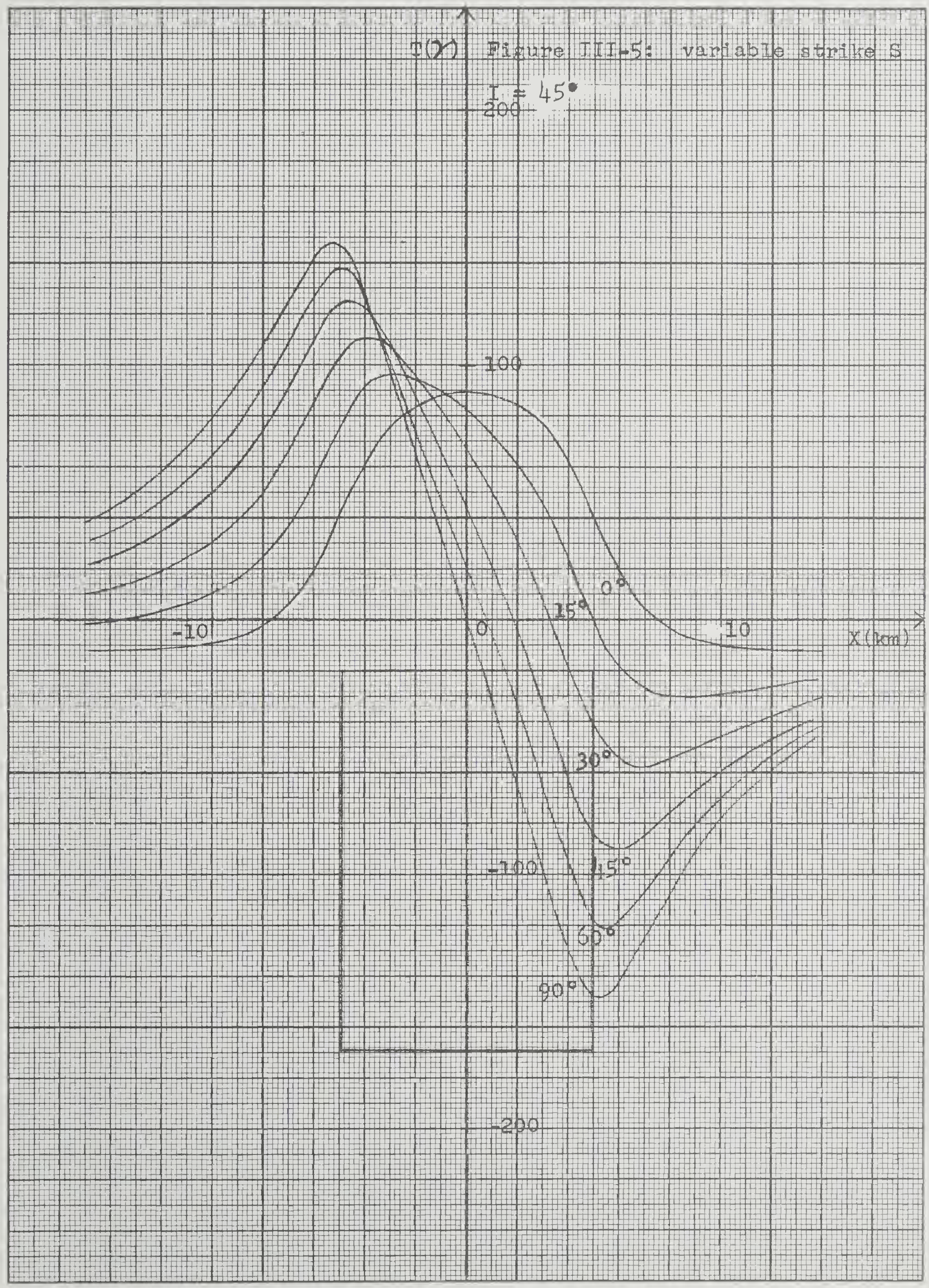




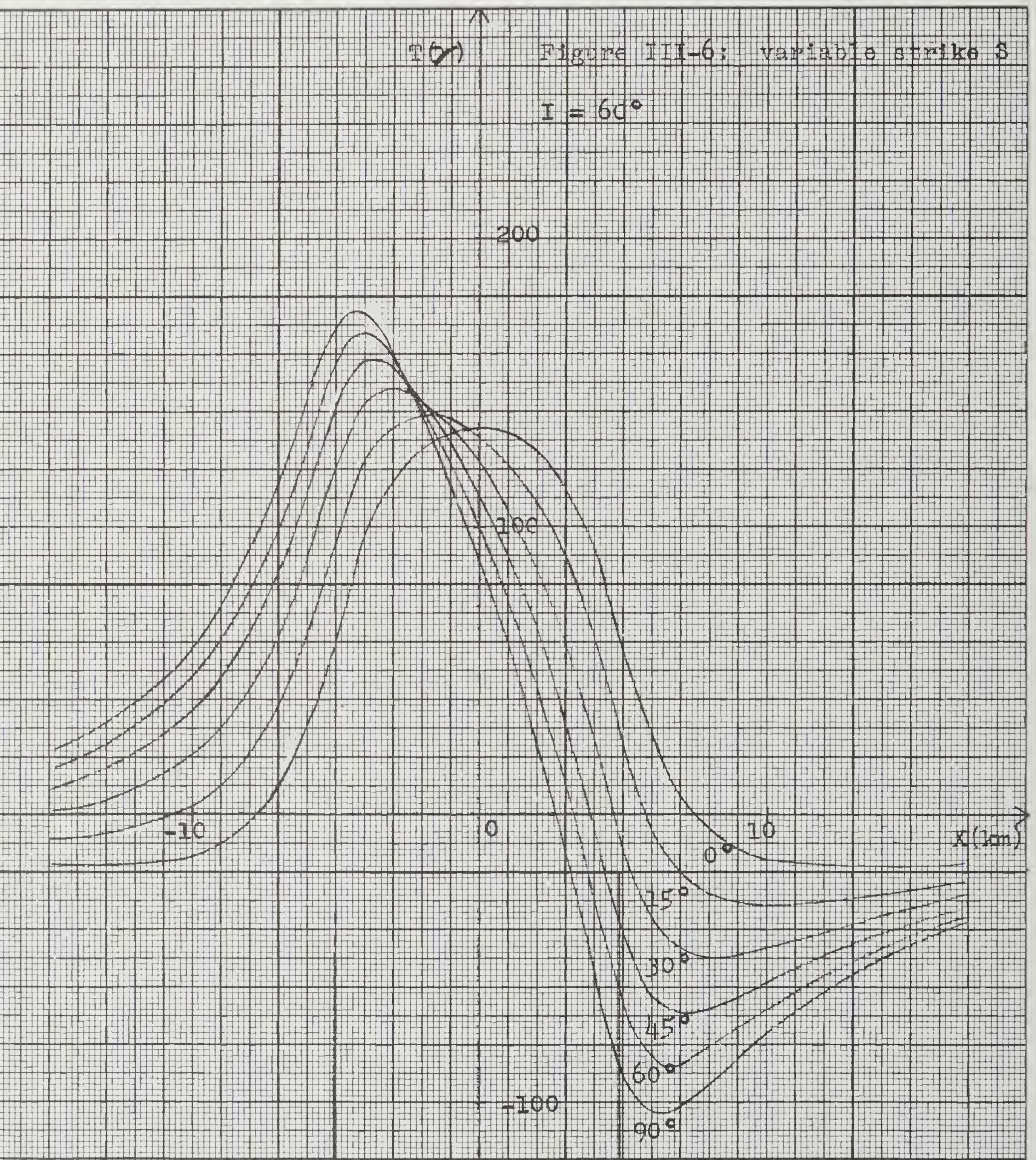




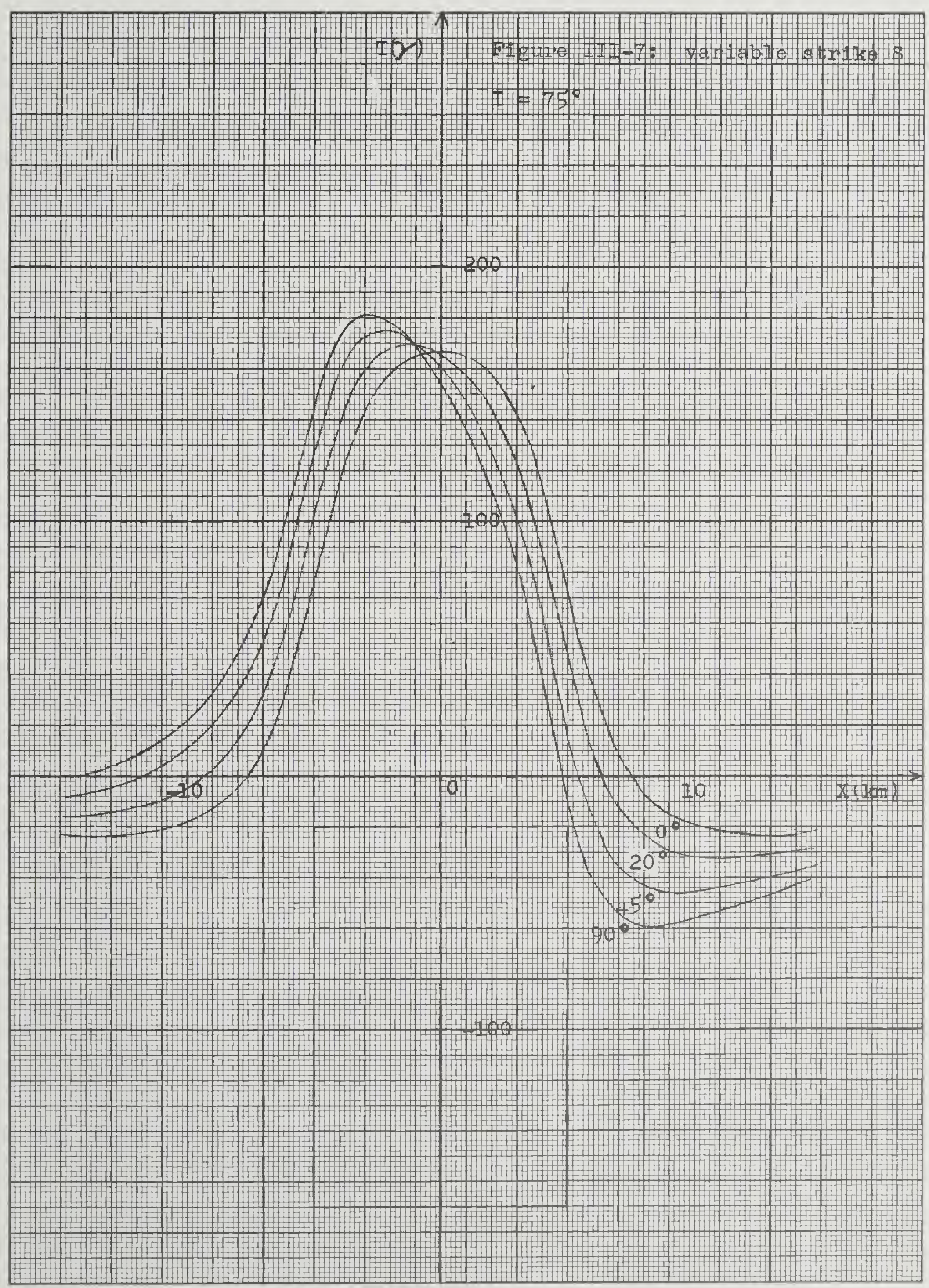




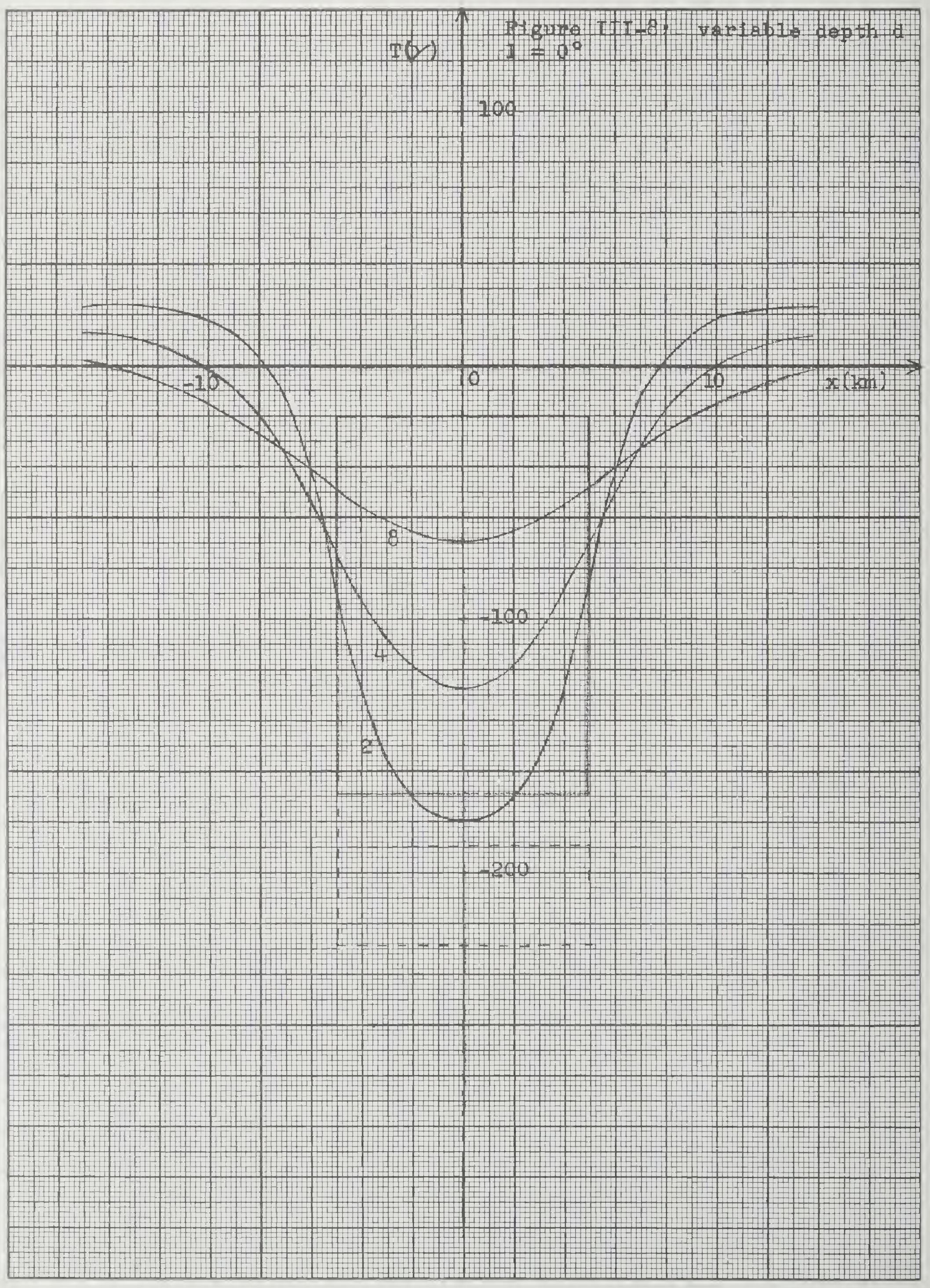




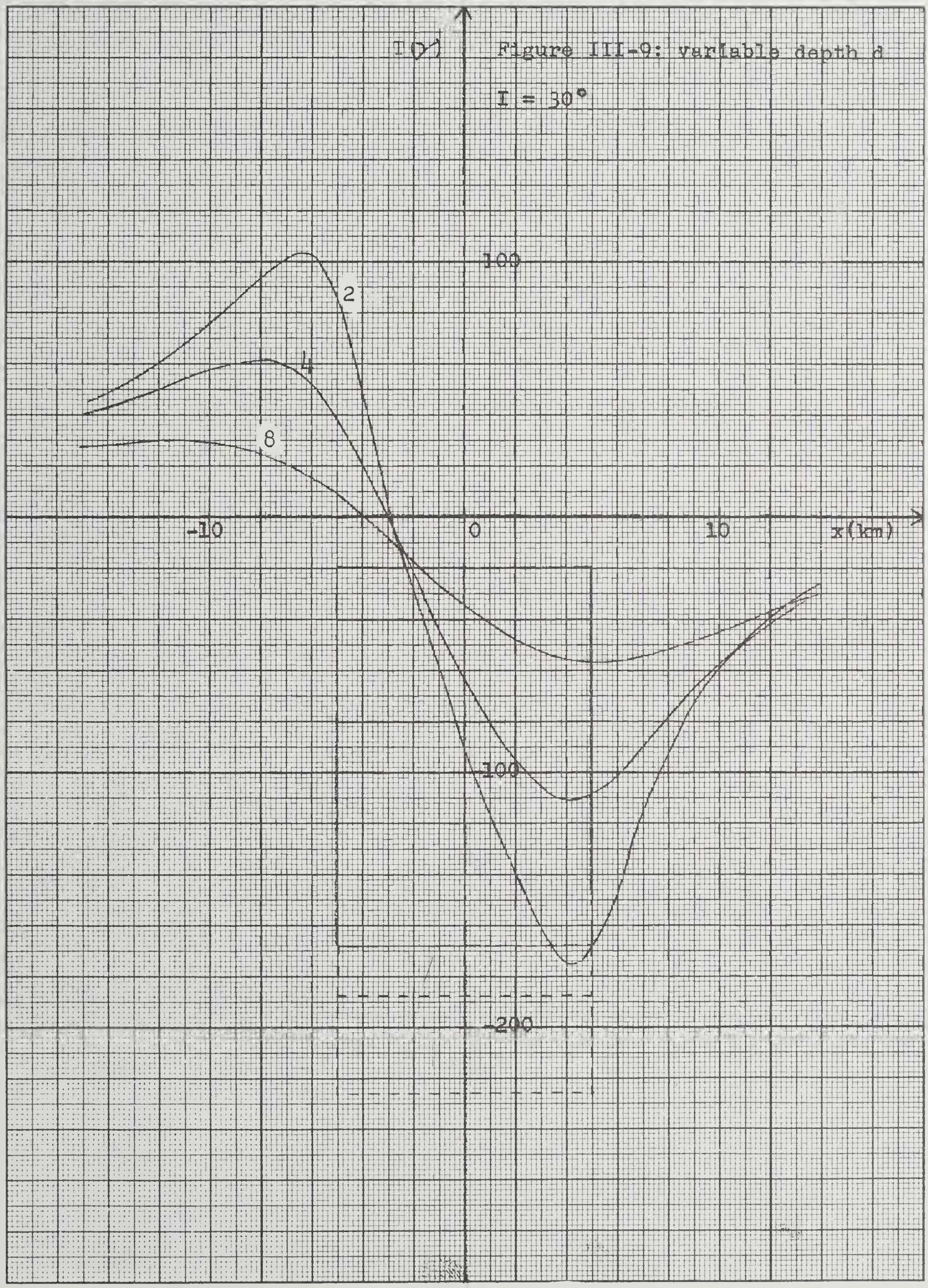




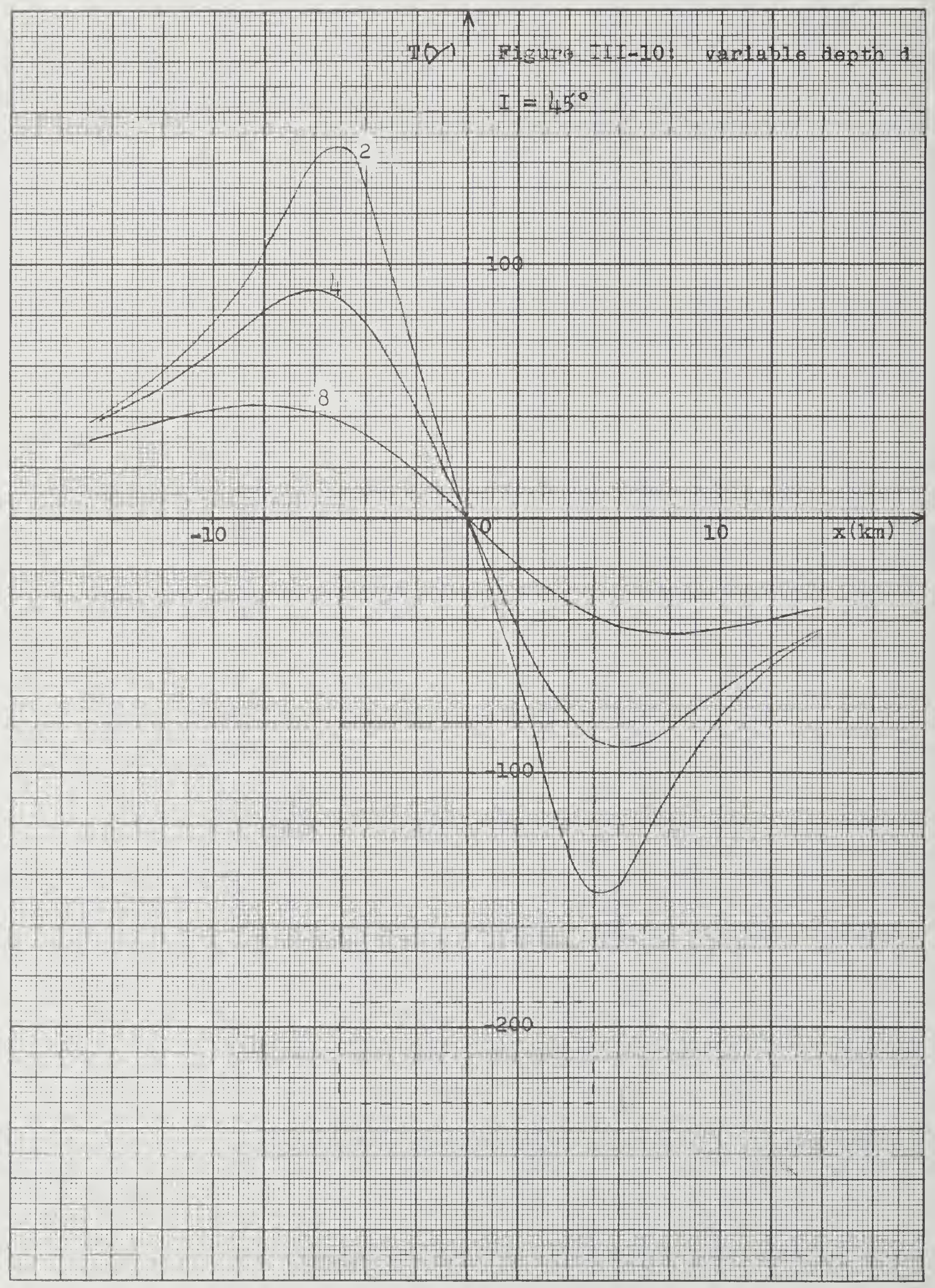




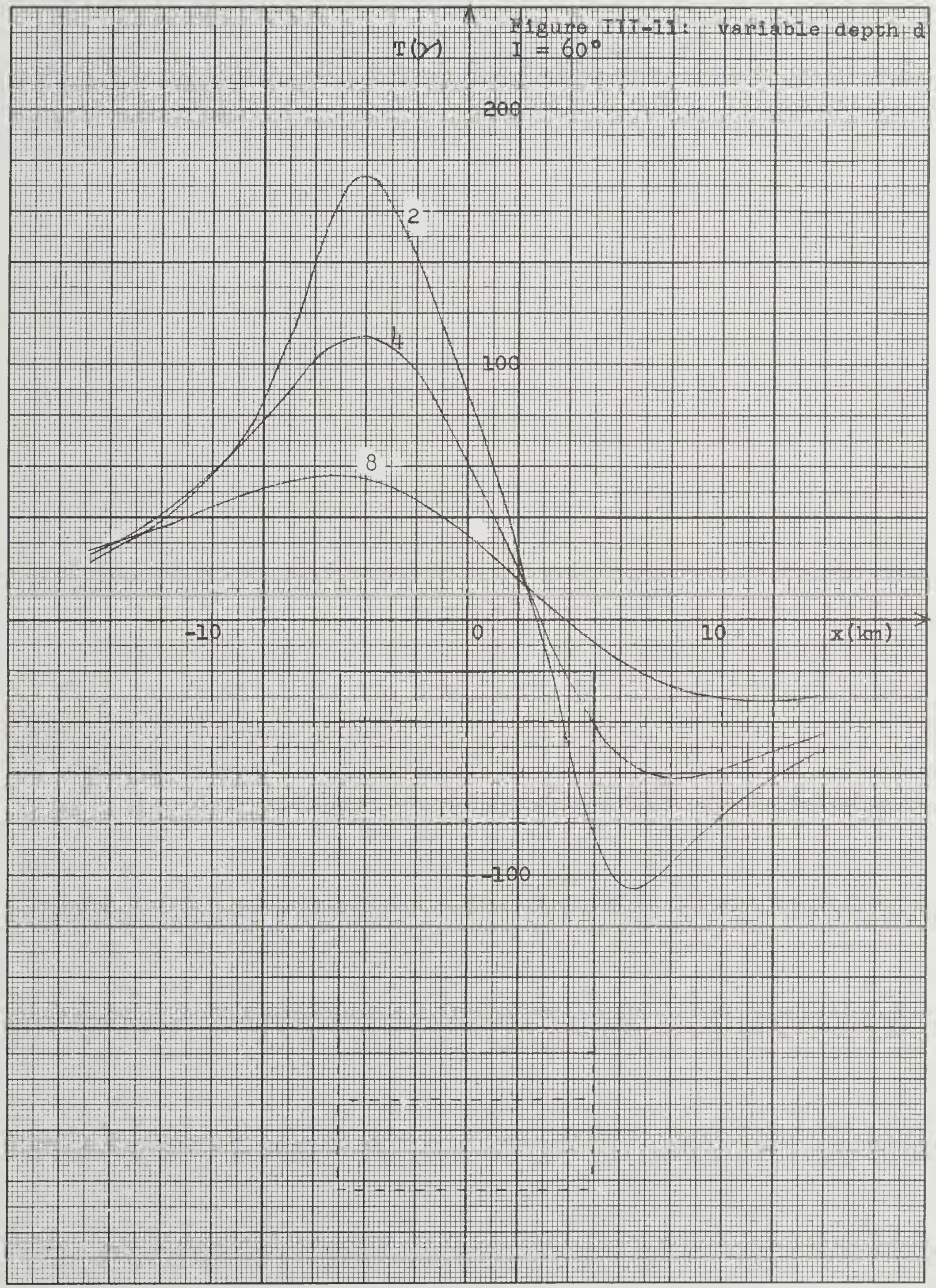




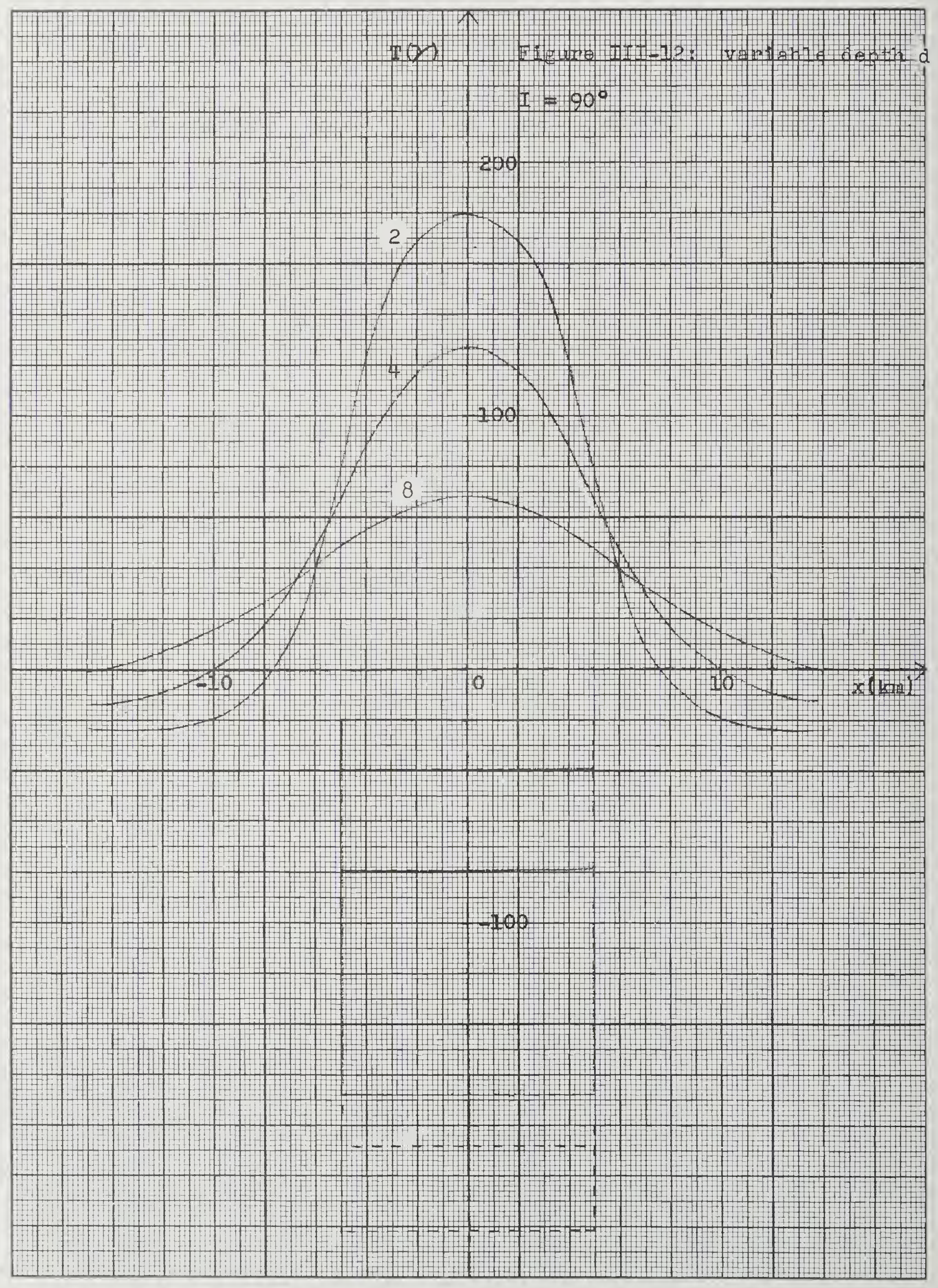




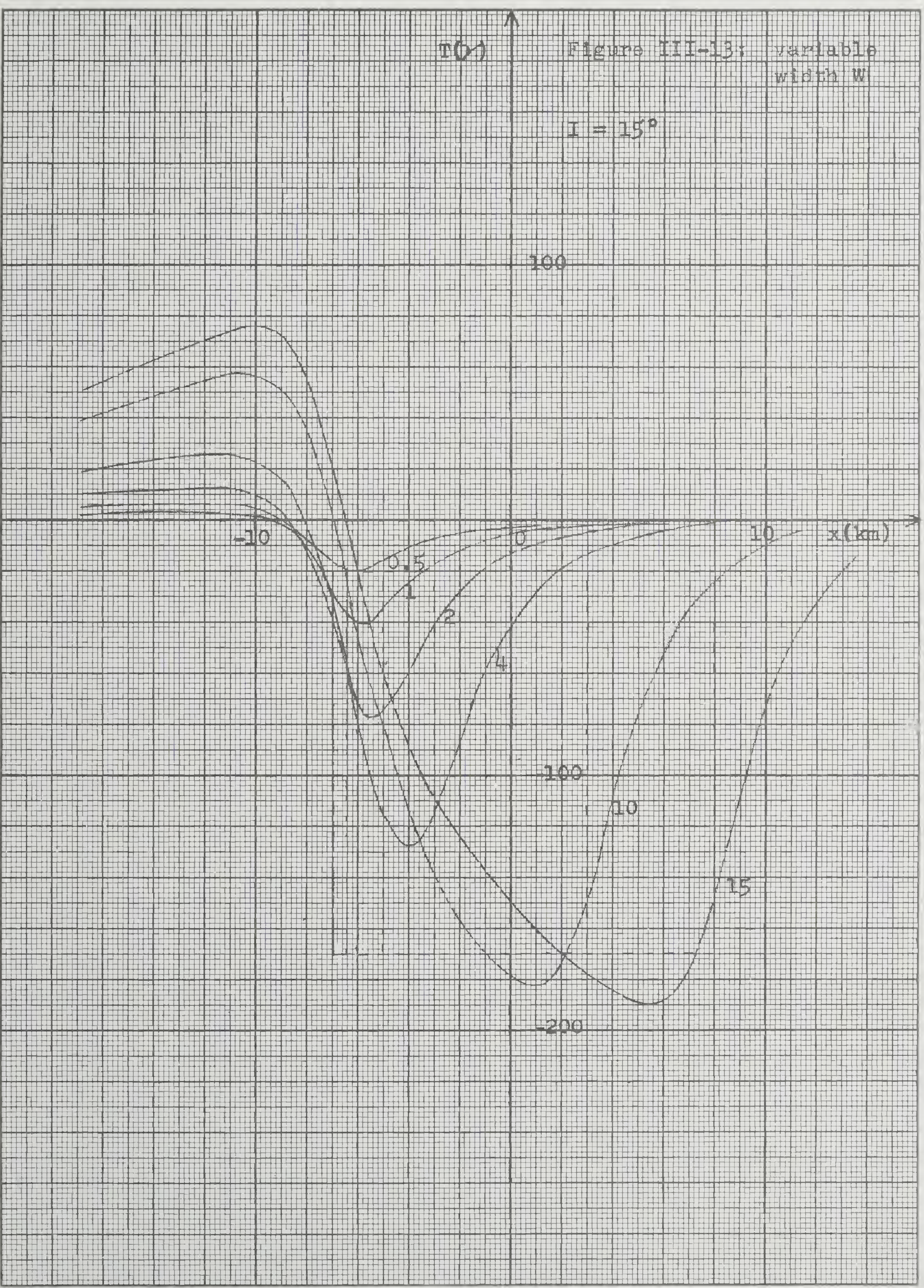




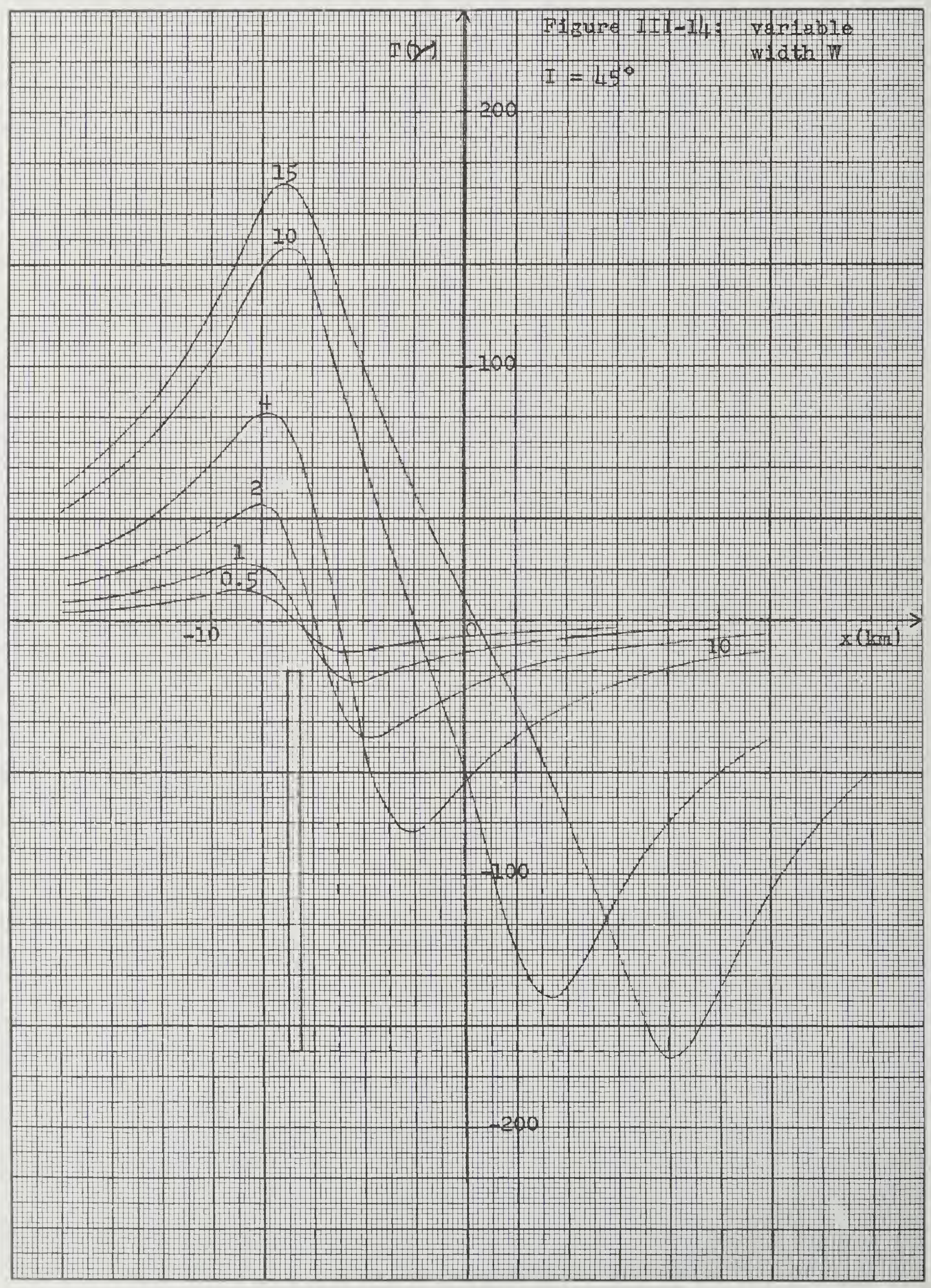




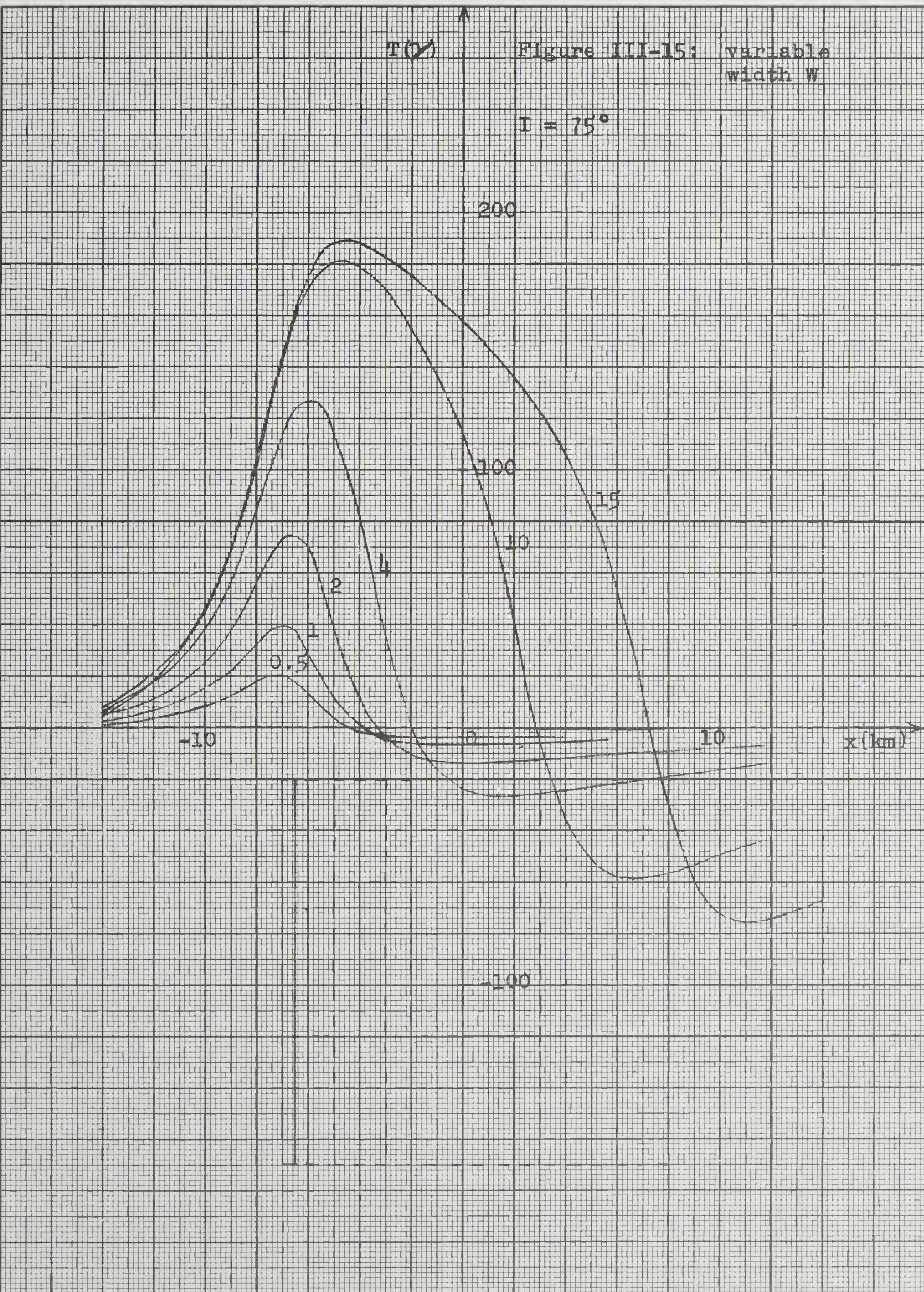




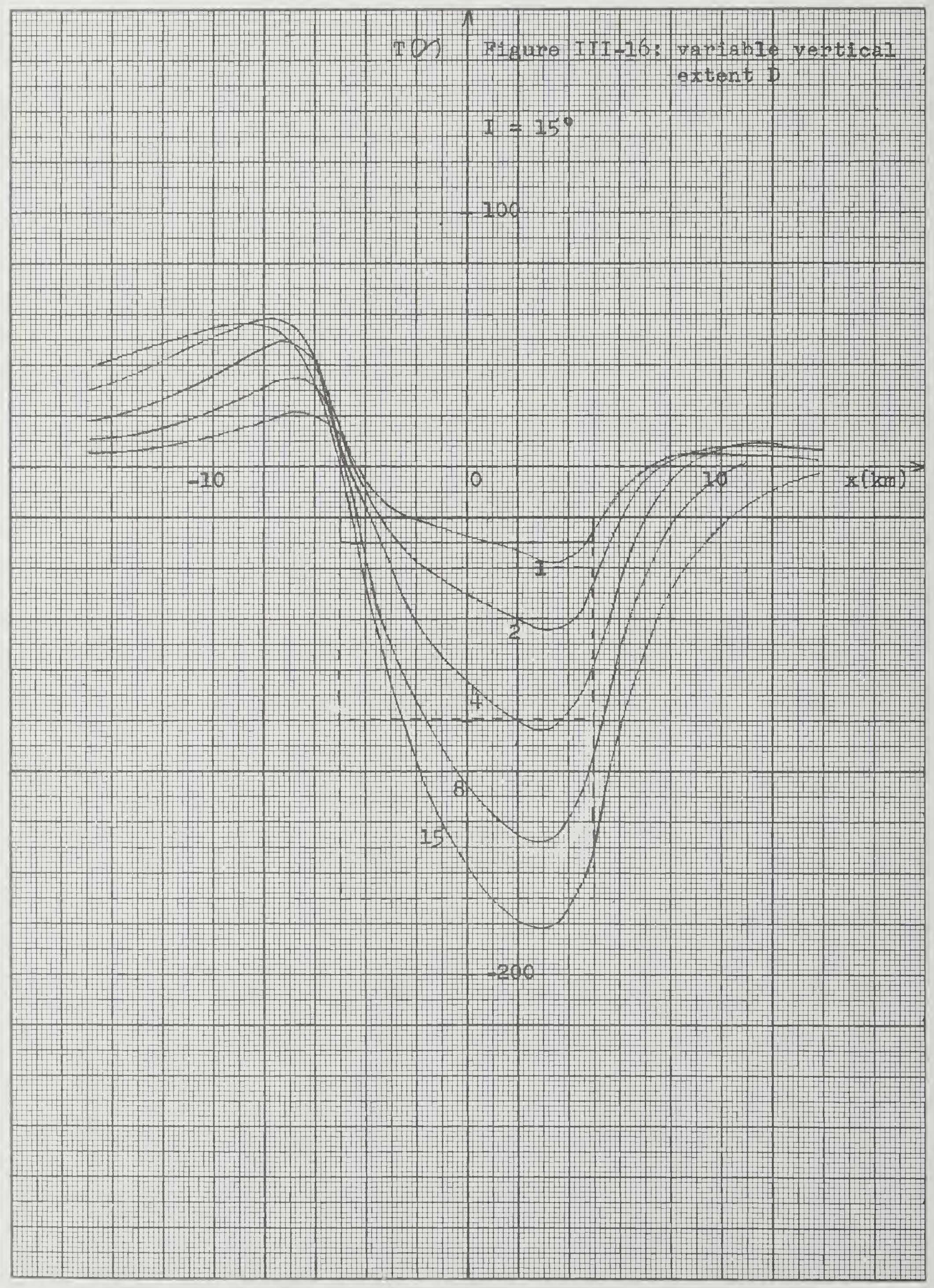




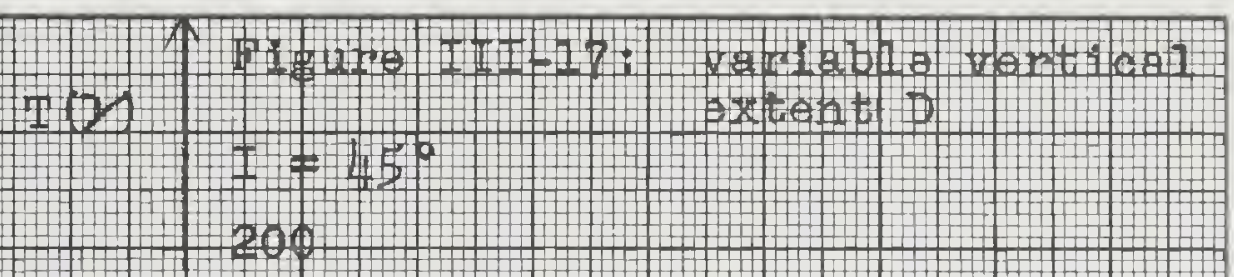

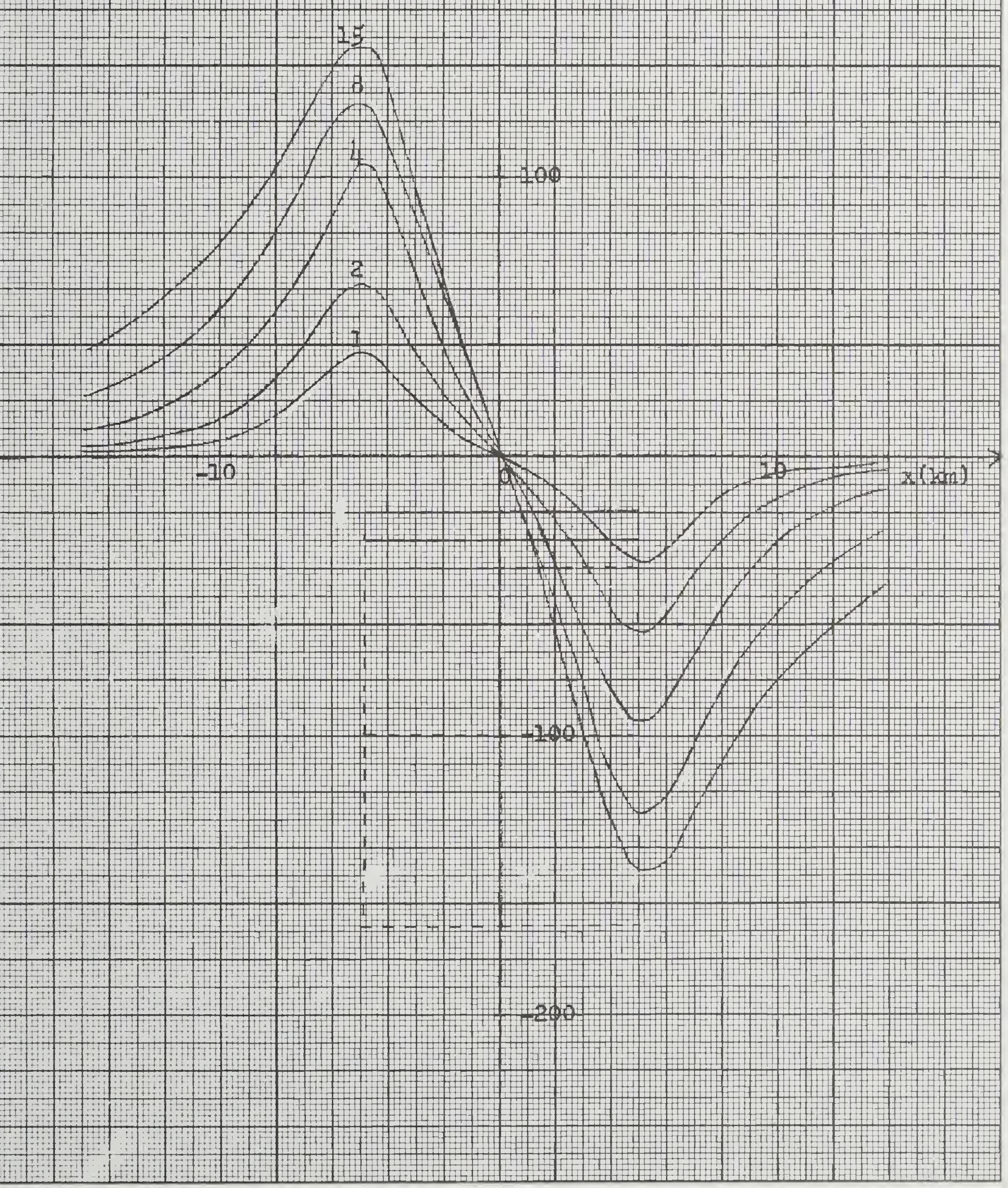




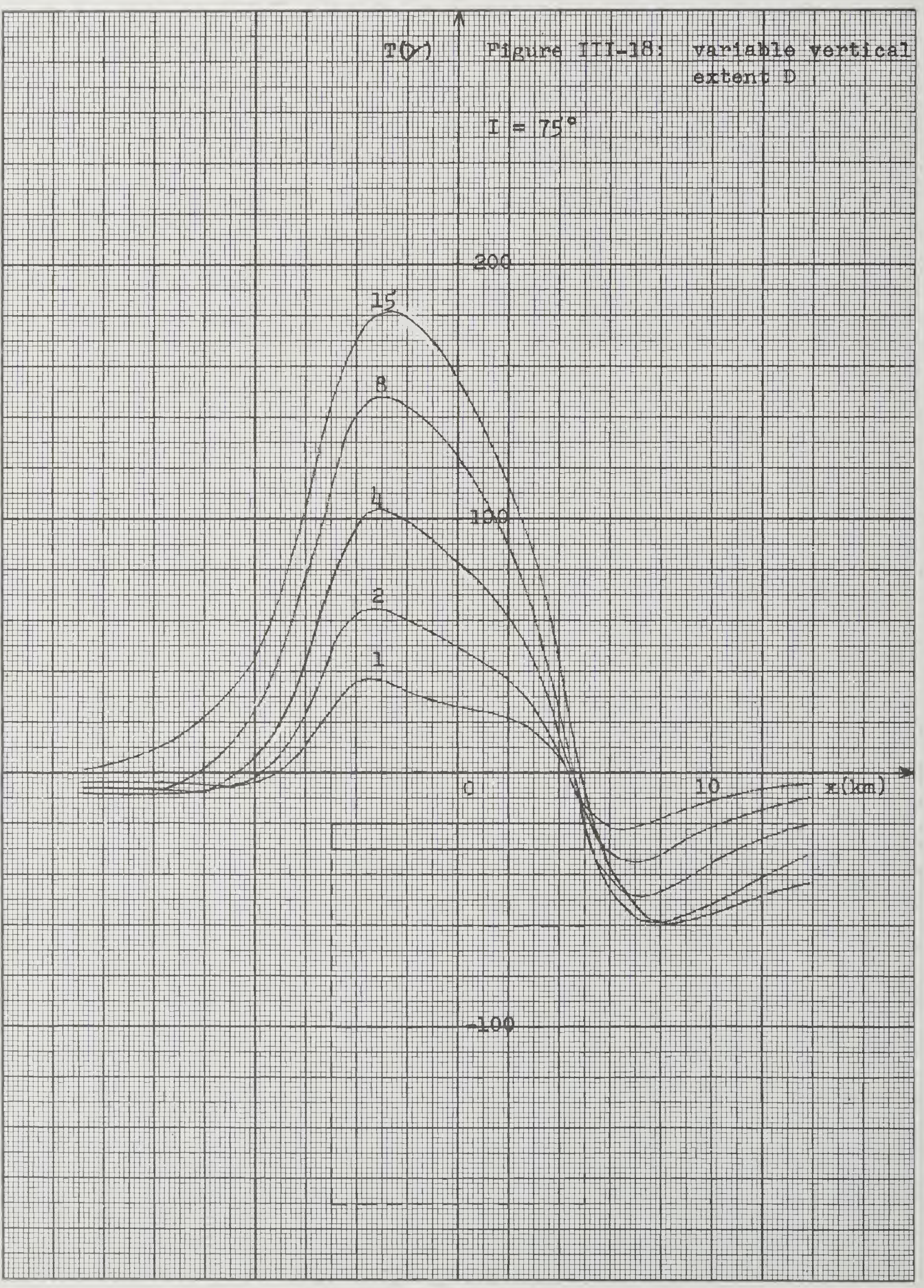




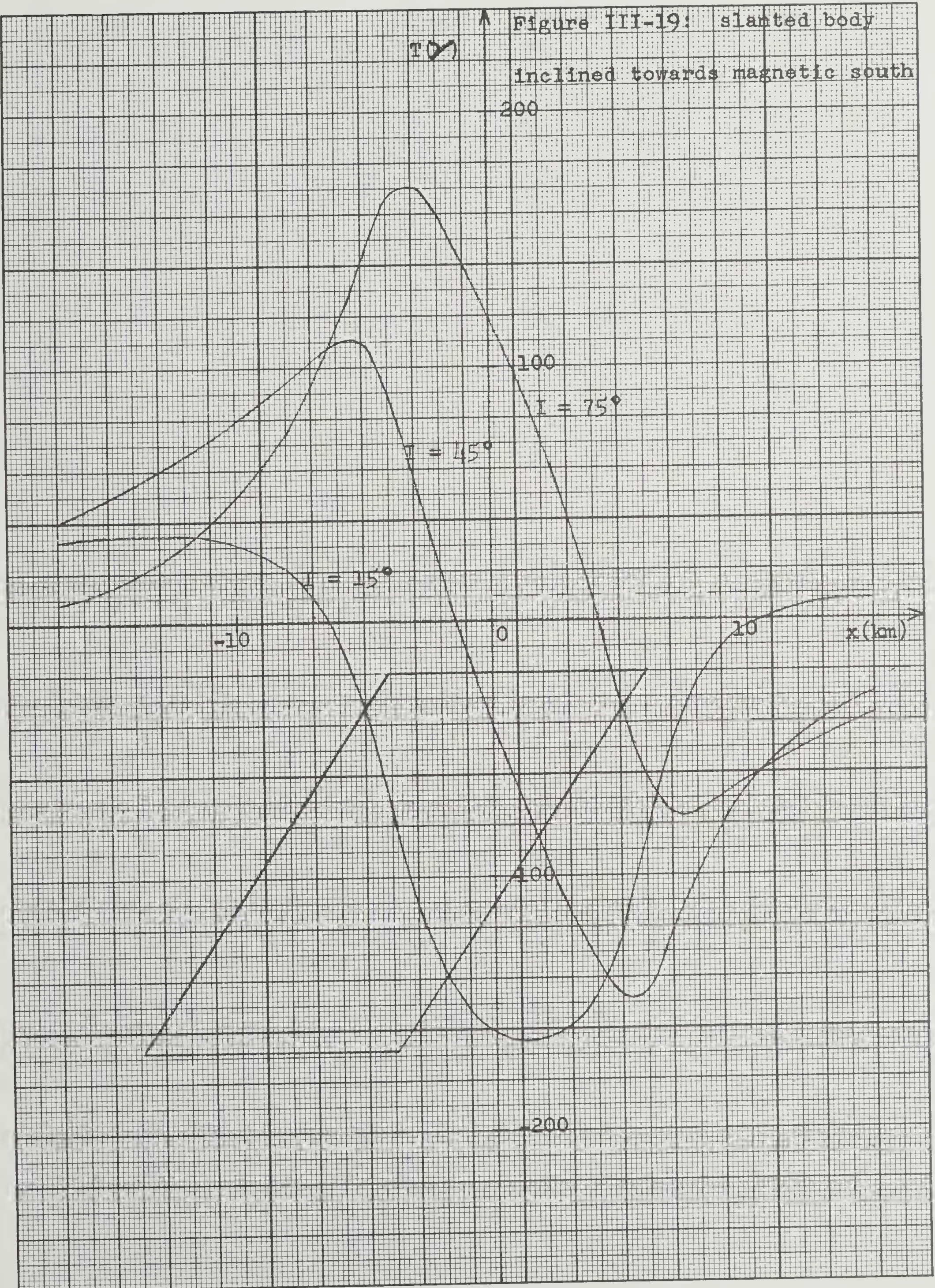




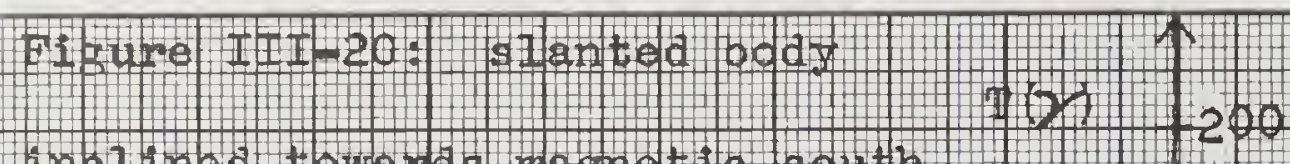
ínelined towards magnetic south

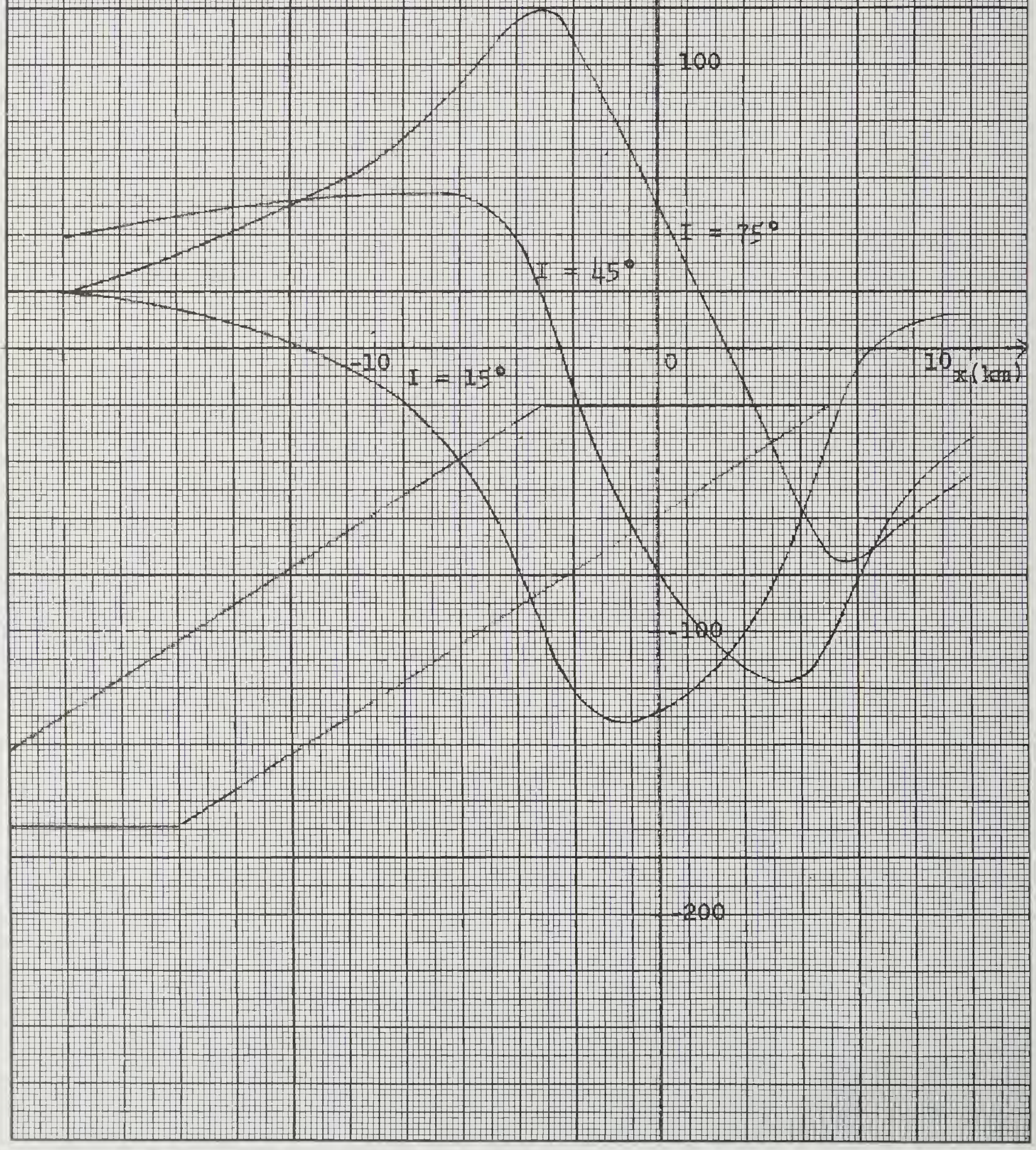




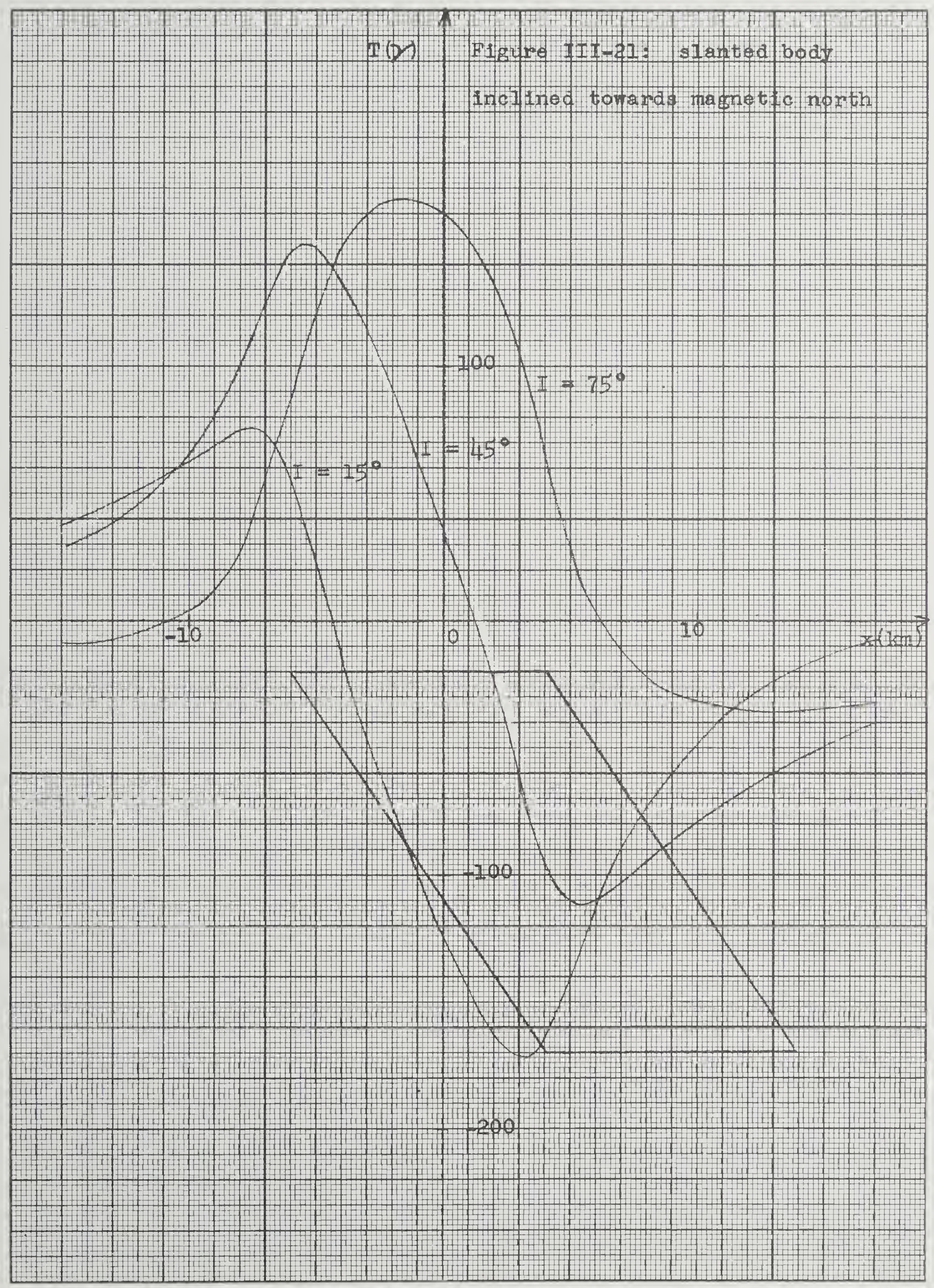




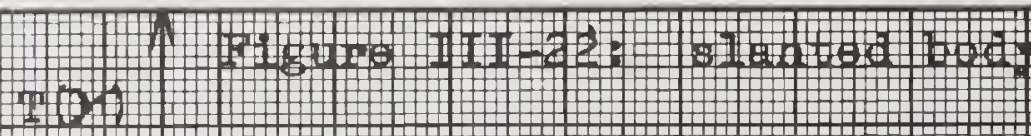
Inc ined towards mangeto num $\mathrm{xob}$

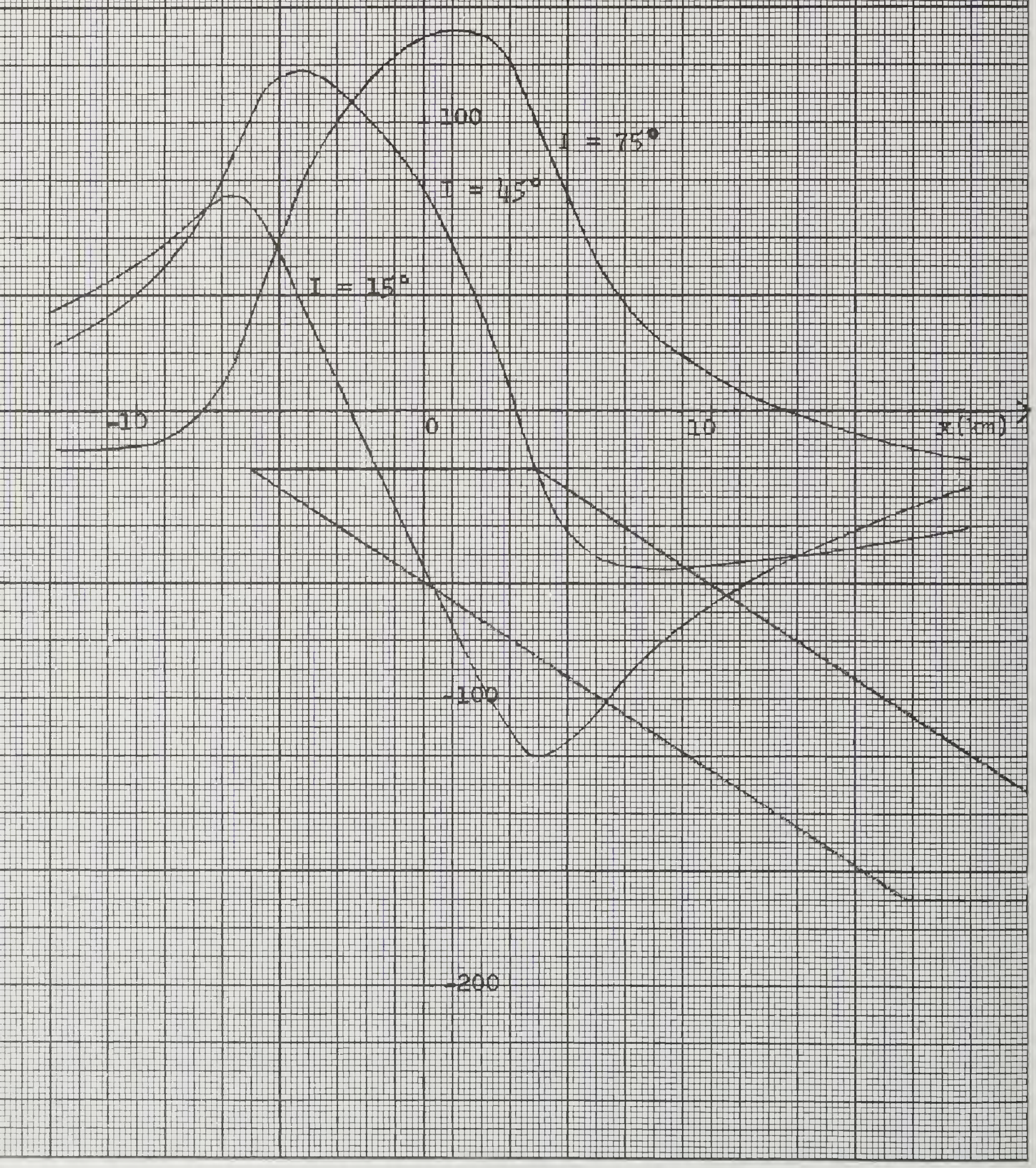




\section{Chapter IV}

A Method of Interpretation for Anomalies of Total Magnetic Intensity caused by

Two-Dimensional Bodies

E.G. Zurfluoh

\section{A. General Remarks}

There are numerous methods of interpretation for magnetic data, each one having a certain range of application. The method of Egyed (1948) which refers specifically to twodimensional bodies depends on the measurement of the horizontal and vertical components and can, therefore, not easily be used for total intensity data.

Among the various existing procedures for the analysis of total intensity anomalies, the most widely used are probably the ones of Vacquier et.al. (1951), Henderson and Zletz (1958) and different mathematical methods as described by Peters (1949).

The mathematical methods are complicated and can only be applied to the interpretation of accurate magnetic maps. The more graphical methods on the other hand have limitations imposed by the geometrical configuration of bodies to be anaIyzed. The method of Vacquier et.81., for instance, becomes inaccurate when applied to bodies with a horizontal extent equal or smaller than its depth of burial. The method of Henderson and Zietz has been partly devised to overcome this difficulty and gives good results for narrow bodies, while it 1s, in tum, not applicable to wide bodies. Both methods relate to prismatic or cylindrical bodies of more or less isometric horizontal cross section. 
Since many of the important geological structures causing magnetic anomalies are much more extended in one direction than in others, the computations of Chapter III are expanded to furnish a quantitative method of analysis for two-dimensional bodies.

It is believed that the method can close a certain gap in the range of graphical methods and it is also considered desirable to work out a simple and uniform method which can be used for bodies of small as well as large width compared to depth of burial. The method has been devised for simplicity of procedure. In order to minimize the influence of adjacent anomalies only distances near the main extreme of the anomaly have been used for the depth determinations. As a general rule it is only feasible to use differences between abscissas of certain points on the curve since all measures involving the total intensity values are dependent on the susceptibility of the body. From various distances that have been considered, the distance $h$ between points of half maximum (or minimum) value has been selected as the main depth measure (see Fig. 4-2). The computations have shown that this distance (hereafter called half-width) depends mainly on depth and width and is comparatively little influenced by other factors.

The distance a has been chosen so that the ratio $\mathrm{b}=\mathrm{h} / \mathrm{a}$ gives a clear distinction between different values of $\mathrm{w} / \mathrm{d}$. In order to satisfy this condition two different distances a had to be used for middle and for low and high magnetic latitudes. For inclinations near $0^{\circ}$ or $\pm 90^{\circ}$ there is a choice between both 
values of a and depending on the circumstances one or the other may give better results.

For the practical applications it is useful to keep the limitations of a method in mind. For this purpose the basic assumptions that have been made are briefly summarized hon:

1. two-dimensional body.

2. vertical sides (this assumption implies that the method is, in general, to be applied to anomalies which are caused by susceptibility contrast and not by topography).

3. vertical extent larger than depth of burial.

4. uniform magnetization.

5. no remanent magnetization (if there is a remanent magnetization parallel to the present earth's field, the method can be used but susceptibility estimates will yield too high values).

\section{B. Mathematical Expressions}

The derivation of a few mathematical expressions for the anomalies to be analyzed will be helpful in evaluating the properties of the computed curves.

1. Formula for the anomaly curve

Assuming induced magnetization only, we obtain the following expression for the total intensity anomaly produced by a twodimensional body with vertical sides and rectangular cross section (see Fig. 4-1) by using equations $(2-7),(2-8),(2-9)$ and the expressions on page 2-10: 
$T=K_{1} A+K_{2} B$

at which

$$
\begin{aligned}
& k_{1}=2 k F\left(\cos ^{2} I \sin ^{2} s-2 n^{2} I\right) \\
& k_{2}=2 k F \sin 2 I \sin s
\end{aligned}
$$

and

$$
\begin{aligned}
& A=\theta_{2}-\theta_{1}-\theta_{2}^{\prime}+\theta_{1}^{\prime} \\
& B=\log \frac{r_{2}}{r_{1}}-\log \frac{r_{2}^{\prime}}{r_{1}^{\prime}}
\end{aligned}
$$

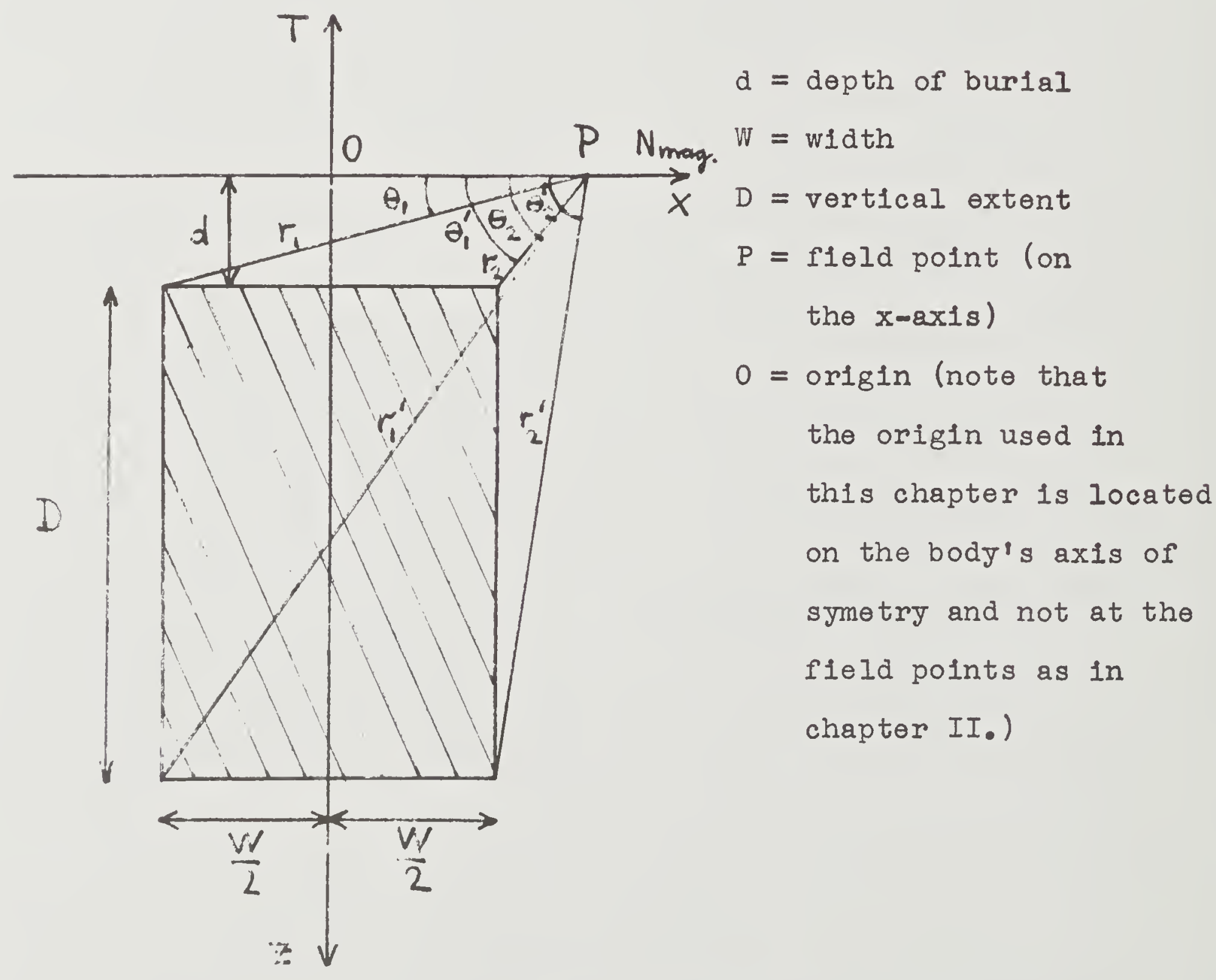

Fig. 4-1: cross section of assumed body and coordinate system. 
Using distances instead of angles and setting $\bar{d}=d+D$ for simplification we obtain the following expressions for $A$ and

B:

$$
\begin{aligned}
& A=\operatorname{arctg} \frac{\bar{d}}{x+\frac{w}{2}}-\operatorname{arctg} \frac{d}{x+\frac{\pi}{2}}-\operatorname{arctg} \frac{\bar{d}}{x-\frac{w}{2}}+\operatorname{arctat} \frac{d}{x-\frac{w}{2}}(4-3) \\
& B=\frac{1}{2}\left[\operatorname { l o g } \left(-j^{2}+\left(x+\frac{w}{2}\right)^{2}-\left(x-1+x+\frac{N}{2} j^{2}\right\}\right.\right.
\end{aligned}
$$

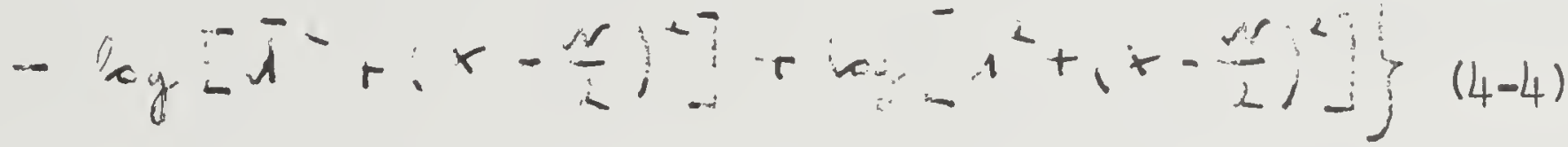

Equation $(4-4)$ can also be written as:

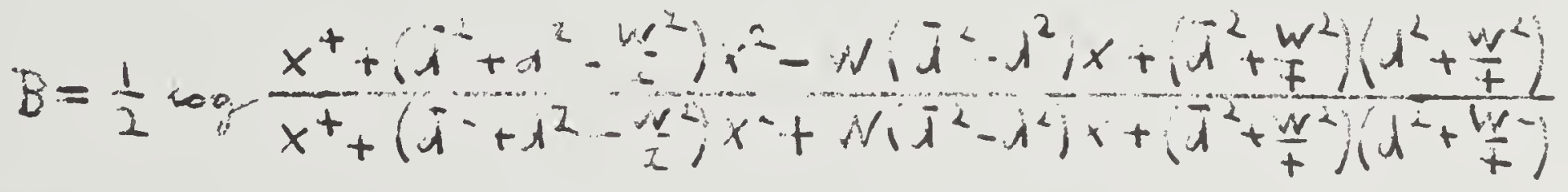

$$
\begin{aligned}
& (4-4 a)
\end{aligned}
$$

Equation (4-I) gives the resolution of the total intensity anomaly into an even and an odd term as will be shown here:

The function $A(x)$ represents the even term since replacing $x$ by $-x$ in equation $(4-3)$ does not change the expression. $B(x)$ can be written as $B(x)=\log f(x)$, where $f(x)$ is the square root of the fraction in equation $(4-4 a)$. If $B$ is odd it must satisfy the condition $\log f(x)=-\log f(-x)$, and therefore, $f(x)=1 / f(-x)$. That this condition is fulfilled can easily be seen from equation $(4-4 a)$

2. Extremes positions

In order to evaluate the maxima and minima positions we take the derivative of formula $(4-I)$

$$
\mathrm{T}^{\prime}=\mathrm{K}_{1^{\prime}} \mathrm{A}^{\prime}+\mathrm{K}_{2} \mathrm{~B}^{\prime}
$$


$4-6$

Taking the derivatives of $A$ and $B$ from equations (4-3) and $(4-4)$ we obtain:

$$
\begin{aligned}
& A^{\prime}(x)=-\frac{\bar{d}}{\bar{d}^{2}+\left(x+\frac{w}{2}\right)^{2}}+\frac{d}{d^{2}+\left(x+\frac{w}{2}\right)^{2}}+\frac{\bar{d}}{\bar{d}^{2}+\left(x-\frac{w}{2}\right)^{2}}-\frac{d}{d^{2}+\left(x-\frac{1}{2}\right)^{2}} \\
& B^{\prime}(x)=\frac{x+\frac{w}{2}}{d^{2}+\left(x+\frac{w}{2}\right)^{2}}-\frac{x+\frac{w}{2}}{A^{2}+\left(x+\frac{w}{2}\right)^{2}}-\frac{x-\frac{w}{2}}{x^{2}+\left(x-\frac{w}{2}\right)^{2}}+\frac{x-\frac{w}{2}}{d^{2}+\left(x-\frac{w}{2}\right)^{2}(4-6)}
\end{aligned}
$$

In a more concise notation the derivative of the total intensity anomaly can then be written as:

$$
\begin{aligned}
& \left.F^{\prime}, r\right)=\frac{K_{1} E^{\prime}+S^{\prime} F^{\prime}}{D_{1} D_{2} D_{2} D^{\prime}} \quad(4-7) \\
& \text { where } E^{\prime}=\bar{d}\left(D_{1} D_{2} D_{4}-D_{2} D_{3} D_{4}\right)+A\left(D_{1} D_{2} D_{4}-D_{1} D_{2} D_{3}\right) \\
& \text { and }==\left(x+\frac{W}{2}\right)\left(D_{2} D_{3} D_{4}-D_{1} D_{3} D_{4}\right)+\left(x-\frac{W}{2}\right)\left(D_{1} D_{2} D_{3}-D_{1} D_{2} D_{4}\right) \\
& \text { Expression }(4-7) \text { is of the form } T^{\prime}(x)=\frac{P_{(x)}^{(5)}}{P_{(x)}^{(x)}}
\end{aligned}
$$

where $P(8)(x)$ and $p(5)(x)$ are polynomials of the eighth and fifth degree respectively.

Setting $T^{\prime}(x)=0, P^{(8)}(x)$ contributes the two solutions $x= \pm \infty$ (where the anomaly curve approaches the $x$ - axis asymptotically), and in the following we will only consider the equation:

$$
P(5)) x)=K_{1} E^{\prime}+K_{2} F^{\prime}=0
$$


Substituting for $D_{1}$ through $D_{4}$ we obtain the following $\theta x-$ pressions for $E^{\prime}$ and $F^{\prime}$ :

$$
\begin{aligned}
E^{\prime}(x)= & -2 W D x^{5}+W D\left(4 \bar{d} d+W^{2}\right) x^{3} \\
& +W\left[2 \bar{d} d\left(d^{3}-d^{3}\right)-4 \bar{d} d W^{2} D-\frac{1}{8} W^{4} D\right] x \\
F^{\prime}(x)= & 3 W\left(d^{2}-d^{2}\right) x^{4}+W\left(d^{2}-d^{2}\right)\left(d^{2}+d^{2}-\frac{W^{2}}{2}\right) x^{2} \\
& -W\left(d^{2}-d^{2}\right)\left[d^{2} d^{2}+\frac{W^{2}}{4}\left(d^{2}+d^{2}\right)+\frac{W^{+}}{16}\right]
\end{aligned}
$$

For the general case it is not possible to obtain an exact solution for equation (4-8). However, sinc $\theta E^{\prime}$ is an odd function

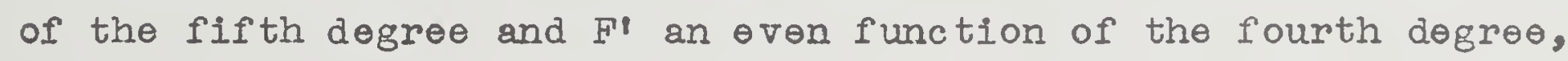
we can solve the problem for cases where either $K_{1}$ or $K_{2}$ is equal to zero.

a. $\mathrm{K}_{2}=0$.

According to equations $(4-2)$ this condition is satisfied for $I=0^{\circ}$ or $\pm 90^{\circ}$ and for $s=0^{\circ}$. Solving the equation $E^{\prime}(x)=0$ we get the following extremes positions:

$$
x_{1}=0 \quad x_{2,3,4,5}= \pm \sqrt{\bar{d} d+\frac{w^{2}}{4} \pm \frac{1}{2} \sqrt{2 d d\left[2(d+d)^{2}-3 W^{2}\right]}}
$$

b. $\mathrm{K}_{1}=0$.

This condition can also be written as $\tan I= \pm \sin s$ (from (4-2). The relation can only be satisfied for $+45^{\circ} \geqslant I \geqslant-45^{\circ}$. Two extreme values are: $I=45^{\circ}, \mathrm{s}=90^{\circ}$ and $I=0^{\circ}, \mathrm{s}=0^{\circ}$. 
For the latter case both $K_{1}$ and $K_{2}$ are equal to zero and no anomaly will be observed.

From the equation $F^{\prime}(x)=0$ we obtain the extremes positions:

$$
x_{1,2,3,4}= \pm \sqrt{\frac{1}{6}\left\{\frac{W^{2}}{2}-\left(d^{2}+d^{2}\right) \pm \sqrt{\left(d^{2}+d^{2}+W^{2}\right)^{2}+12 d^{2} d^{2}}\right.}
$$

for $w \gg d, D$ the expression reduces to: $x= \pm W / 2 \quad(4-12 a)$

\section{Summary}

Formulas have been derived for anomaly curves over twodimensional, rectangular and vertical sided bodies and for the positions of the extremes of the curves for special cases of inclination and strike. It is not possible to obtain exact solutions for some other quantities like the half-widh that are used in the method of analysis presented here.

However, the formulas give information about the general properties of the anomaly curves and they also allow conclusions about the relationship between the different parameters involved. Many of the relations mentioned here are illustrated in a qual1tative way by the curves shown in Chapter III of this report.

The following main conclusions can be pointed out:

The parameters $k$ and $F$ are merely multiplying factors and have no influence on the shape of the anomaly curve (see equations $(4-1)$ and $(4-2))$.

The angular parameters I and s determine the relative importance of the even and odd terms of the curve. From equations (4-2) it follows that for positive and negative inclinations of 
equal magnitude the anomaly curves are symmetric with respect to the z-axis, if the other parameters are held constant. This is true if the origin of the coordinate system is taken over the center of the body, and the profiles are drawn in the standard way with the northerm end to the right.

It can be shown that for $s=90^{\circ}$, constant body parameters and for inclinations that differ from $45^{\circ}$ by equal and opposite amounts, there is a central symmetry with respect to the origin. Mathematically this can be formulated:

$$
I=45^{\circ} \pm \alpha \quad T(x, \alpha)=-T(-x,-\alpha) \text { for } s=90^{\circ}
$$

The two symmetry relations (se日 also page 2-9) reduce the amount of computation necessary for the method of interpretation. Since we can account for different strikes by applying a correction to the values computed for $s=90^{\circ}$ ( $s e \theta$ page 4-43), we can restrict ourselves to computing curves for inclinations between $0^{\circ}$ and $+45^{\circ}$.

The first solution of equation $(4-12)$ gives the distance of the point with maximum value from the origin which coincides with a zero position of the anomaly curve for the cases where $K_{I}=0$. Th1s is the distance a which is used for the interpretation at certain inclinations (see Fig. 4-2). The formula shows that for great width it is approximately equal to half the body's width and that, therefore, the main extremes are located over the edges of the body.

With respect to the body parameters $d, W$ and $D$ it can be sald that, as test computations have shown, changes in $D$ in 
general affect mainly the amplitude of the anomaly, while they have less influence on the location of the extremes and of the zero positions. Furthermore, for physical reasons it is clear that if $D$ is greater than a certain value, say greater than d or $W$, an increase in $D$ will only slightly affect the anomaly curve because the added magnetized mass is too distant from the plane of measurement.

\section{Description of Method}

The quantitative analysis of total intensity anomalies described below is based on theoretical curves which have been computed for rectangular and vertical sided two-dimensional bodies (see Fig. 4-1). Sets of curves have been obtained for fixed ratios of width to depth by varying denth and width proportionally (Figs. 4-13. to 4-26). In addition the curves for variable strike of Chapter III (Figs. 3-3 to 3-7) are used. The analysis can be carried out in the following steps:

1) The inclination $I$ of the region is determined by some means (isogonic maps, tables or measurements).

2) The approximate strike $s$ of the body causing an anomaly is determined from the magnetic or geological map or from other data available.

If no information of this kind is at hand a rough estimate of $\mathrm{s}$ can sometimes be obtained by comparing the measured anomaly with the curves with variable strike in Chapter III.

3) If a magnetic map is to be interpreted, a profile is 
drawn through the center of an anomaly at right angles to its strike.

If only profiles have been measured, the profile has to be projected on the diroction perpendicular to the strike. For the practical application it is sufficient to multiply the distances $h$ and a (see below) by the sine of the angle between profile and strike.

4) The profile then is compared with the computed curves and a curve that most closely resembles the actual on is chosen, while keeping in mind the changes in shape produced by the strike.

Since often the exact regional field is not known, the main purpose of this comparison is to determine a zero line for the anomaly.

5) From the measured anomaly profile the distances $h$ and $a$ and the ratio $b$ are determined from the main extreme of the curve (see Fig. 4-2). It is essential that the part of the anomaly which lies between the main maximum and main minimum be used for the determination of $a$.

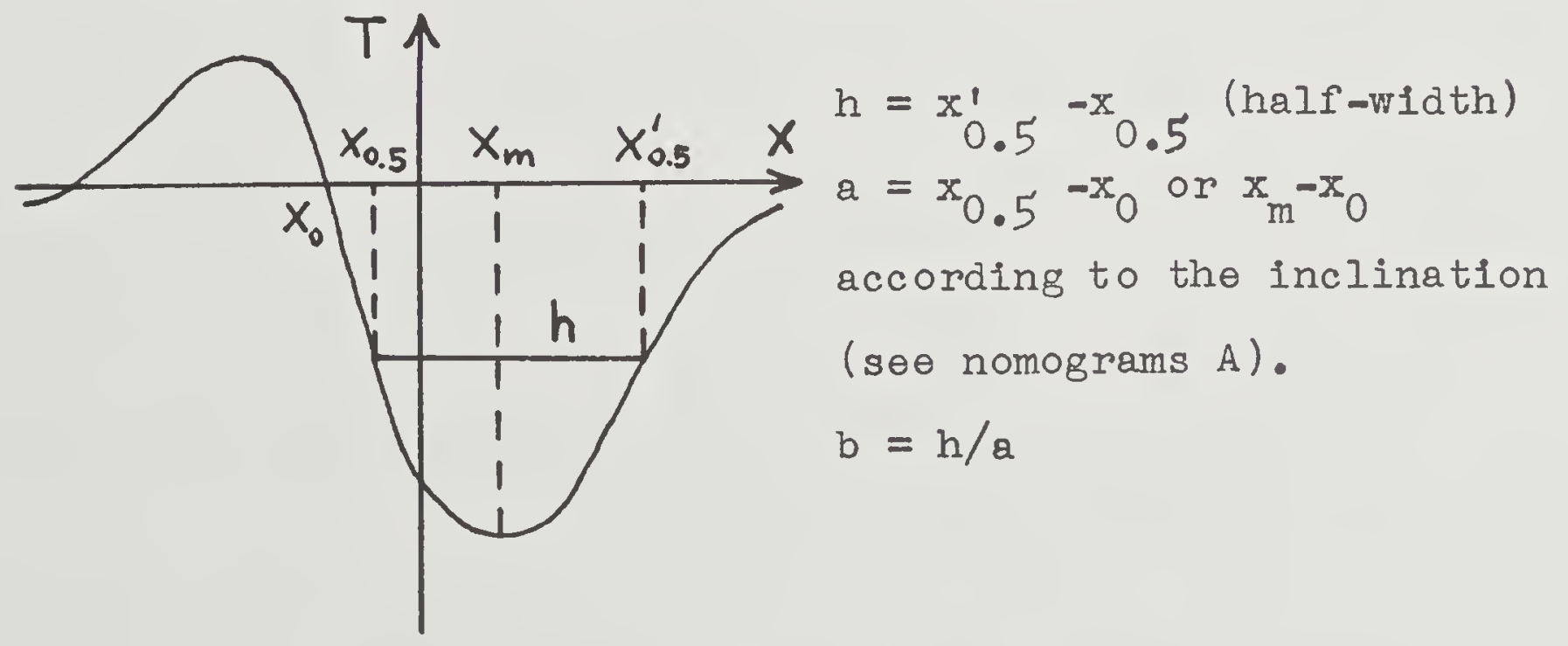

Fig. 4-2: Total intensity anomaly and explanation of $h, a$ and $b$. 
6) If the strike s differs substantially from $90^{\circ}$ a correction has to be applied to $h$ and $b$ by using tables 4-1 to 4-4 (page 4-55).

7) With the corrected values of $h$ and $b$ enter nomogram $A$ for the nearest inclination. (Figs. 4-28 to 4-37). Read a ratio of $\mathrm{W} / \mathrm{d}$ for the body.

8) Nomogram A also gives an estimate of the depth of burial d. A more accurate depth estimate can be obtained by using nomogram $B$ with $h$ and the previously determined value of $W / d$. The width $W$ of the body can then be calculated from $d$ and $w / d$.

9) For inclinations near the middle of the $15^{\circ}$ intervals. of the computed curves an interpolation is necessary. For this purpose a W/d - ratio is determined from nomogram A for the two adjacent inclinations. Then the average of the two $W / d$ - ratios is taken and a depth d determined from nomogram $B$ for both inclinations. The average of these two values gives the depth.

10) The susceptibility $k$ of the rock body can be calculated from the maximum value of the anomaly. The model anomalies have been computed for $k=10^{-3}$ emu and a total field strength of 50,000 gammas. After having corrected for the total intensity of the earth's field in the area, we obtain the susceptibility in electromagnetic CGS-units by the following relationship: 


\section{4-13}

$$
k=10-3 \mathrm{~T} \max / \mathrm{T}^{\prime} \underline{\max }
$$

\section{where $T \max =\operatorname{maximum}$ value of measured anomaly $T^{\prime} \max =\operatorname{maximu}$ value of computed anomaly}

It has to be borne in mind, however, that the vertical extent $D$ and the strike $s$ have a strong influence on the amplitude of the anomaly, and therefore, affect the susceptibility measurements.

The interpretation procedure can be clarified by stating an example. The profile in Fig. 4-3 has been computed for an inclination of $26^{\circ}$ and the true body parameters are indicated in the figure.

The half-width $h$ taken from the profile is $6.5 \mathrm{~km}$, the distance $a=x_{m}-x_{0}$ is $4.2 \mathrm{~km}$ and the ratio $b$, therefore, equals 1.55. Correcting for the strike according to table $4-1$ we add $4 \%$ to the half-width and obtain $h=6.8 \mathrm{~km}$. Similarly the corrected value of $b$ is 1.44 .

Entering nomogram $A$ on page 4-51 with the adjusted values of $h$ and $b$, we estimate $a w / d$ - ratio of 3.3. With this and with h nomogram $B$ on page $4-52$ gives us the estimated depth of the body which is approximately $2 \mathrm{~km}$.

In order to complete the interpretation it is necessary to determine the location of the body's center. This can be done in a more or less qualitative fashion as follows: 


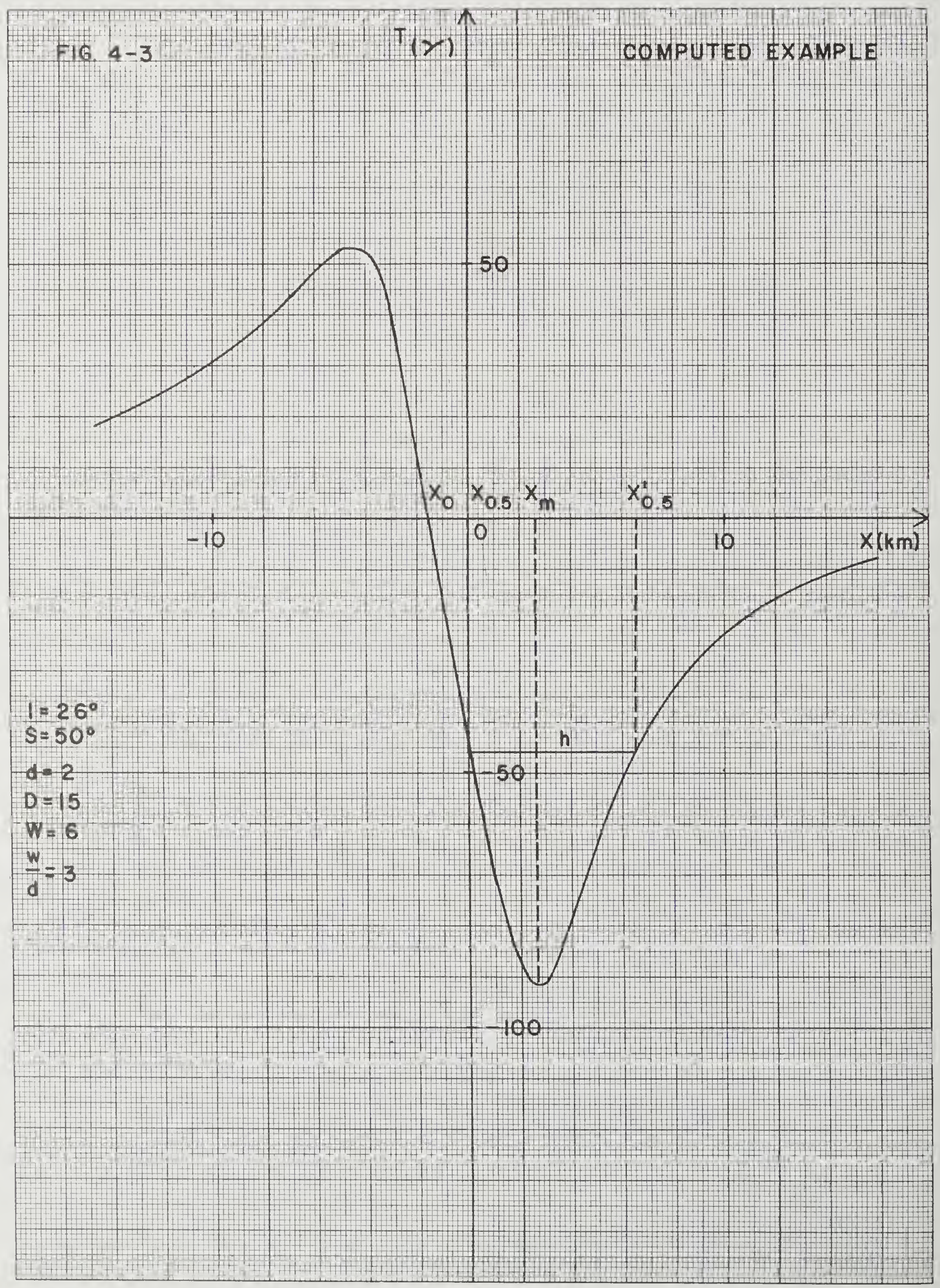




\section{$4-15$}

According to eq. (4-II) for $I=0^{\circ}$ or $\pm 90^{\circ}$ and for $s$ $=90^{\circ}$ the anomaly is symmetric with respect to a vertical Ine through the center of the body and the main extreme is located over the center.

For the cases where the anomaly curve represents an odd function (in the standard coordinate system, see eq. (412). The maximum and minimum are located approximately over the edges of the body, while its center is given by the zero position between them.

For other inclinations and strikes the center of the body can be found between the main extreme and zero positions by interpolation according to the computed curves.

\section{Results of Practical Applications}

1. Analyzed Cases

Some anomalies on Canadian aeromagnetic maps have been interpreted with the method described above. From geological maps it can be inferred that the chosen anomalies are caused by rocks exposed on the surface. The depths determined, therefore, can be directly compared with the flight altitudes. Since no susceptibility values measured from rock samples were imnediately avaliable, no susceptibilities have been calculated for the examples.

Fig. 4-4 to 4-10 show parts of the aeromagnetic maps with the analyzed anomalies. The chosen profiles are indicated 
by the lines $A B$ and the distances $h$ and a are also shown in the figures. The zero level used is given by the point 0 on the profiles. The inclination and the strike for each case are indicated in parenthesis in the accompanying text.

a. Port Hawkesbury, N.S. (Fig. 4-4)

The ratio of $\mathrm{W} / \mathrm{d}$ was found to be approximately 7 and the depth determined is $290 \mathrm{~m}\left(I=73^{\circ}, \mathrm{s}=57^{\circ}\right)$. The flight altitude is about 1000 feet or $305 \mathrm{~m}$ above ground. The geological map shows an outcrop of Pre-Carboniferous granitic rocks surrounded by Mississippian sediments at the location of the anomaly.

b. Deskenatlata Lake South, NW Territories

For the anomaly near the Taltson River (Fig. 4-5) a W/d of 8 and a depth of $290 \mathrm{~m}$ were obtained $\left(I=81^{\circ}, \mathrm{s}=\right.$ $\left.22^{\circ}\right)$.

The anomaly $A_{1} B_{1}$ in Fig. $4-6$ yielded a W/d ratio of 4 and a depth of $225 \mathrm{~m}\left(I=81^{\circ}, \mathrm{s}=13^{\circ}\right)$, the profile $\mathrm{A}_{2} \mathrm{~B}_{2}$ a $W / d$ of 2.5 and a depth of $320 \mathrm{~m}\left(I=81^{\circ}, \mathrm{s}=10^{\circ}\right)$.

The flight altitude for all three cases is 1000 feet or $305 \mathrm{~m}$. The average of the three depths determined is $278 \mathrm{~m}$ which differs less than 10\% from the average flight altitude. Geologically the area is composed of Archaean and Proterozoic acid rocks.

Since $75^{\circ}$ is the nearest inclination and because the 
graphs for $I=75^{\circ}$ are more reliable for small depths than the ones for $I=90^{\circ}$, no interpolation was attempted and only the graphs for $I=75^{\circ}$ were used for these cases.

c. Mack Township, Ontario (Fig. 4-7)

With a W/d ratio of 2 we estimate the depth to be $170 \mathrm{~m}$ ( $I=76^{\circ}, \mathrm{s}=90^{\circ}$ ). The average flight altitude is 500 fe日t or $153 \mathrm{~m}$ above ground. The rocks of the area are Huronian quartzites with quartz diabase and quartz norite intrusions.

d. Gladstone Township, Ontario (F1g. 4-8)

The estimated depth $1 \mathrm{~s} 130 \mathrm{~m}$ and the $\mathrm{W} / \mathrm{d}$ ratio $I$ ( $I=$ $76^{\circ}, \mathrm{s}=55^{\circ}$ ). The geological map indicates Huronian sediments of the Gowganda Formation with intrusives of the same type as found in Mack Township.

- Bright Township, Ontario

The profile in Fig. $4-9$ gives a W/d of 1.3 and a depth of $120 \mathrm{~m}\left(I=76^{\circ}, \mathrm{s}=90^{\circ}\right)$. The results for Fig. 4-10 are: $W / d=1 / 2$ or smaller and $d=170 \mathrm{~m}\left(I=76^{\circ}, \mathrm{s}=90^{\circ}\right)$.

Pre-Huronian granite gnelss and related plutonic rocks are the rock formations encountered near the anomalies.

The average value of all depth estimates for the examined anomalies in Ontario (which are relatively close together) is $148 \mathrm{~m}$, which comes very close to the average flight altitude of $153 \mathrm{~m}$. 


\section{Conclusions}

The test cases have show that the method gives reliable depth estimates for anomalies that are reasonably elongated In shape. For carefully chosen anomalies the results often are accurate to within $10 \%$, although only very small depths of burial have been considered. For greater depths it is to be expected that the method will give even better results, because deviations from the basic assumptions (uniform magnetization etc.) will be less important for those cases.

For this method, like for others, it is of advantage to choose isolated and simple anomalies. As a general criterion it can be said that good results can be expected, if the shape of the anomaly profile is in its major parts comparable to the theoretical curves.

For anomalies which are nearly isometric in the map projection, the depth estimates obtained with this method tend to become too small, because in this case the bodies considered are not two-dimensional. However, if an anomaly of more or less elliptic shape is about three times as long as broad, the method can be applied successfully.

It was not possible to check the $\mathrm{W} / \mathrm{d}$ ratios determined for the actual anomalies considered above. But measurements on computed curves have shown that they are somewhat less reliable than the depth estimates. 


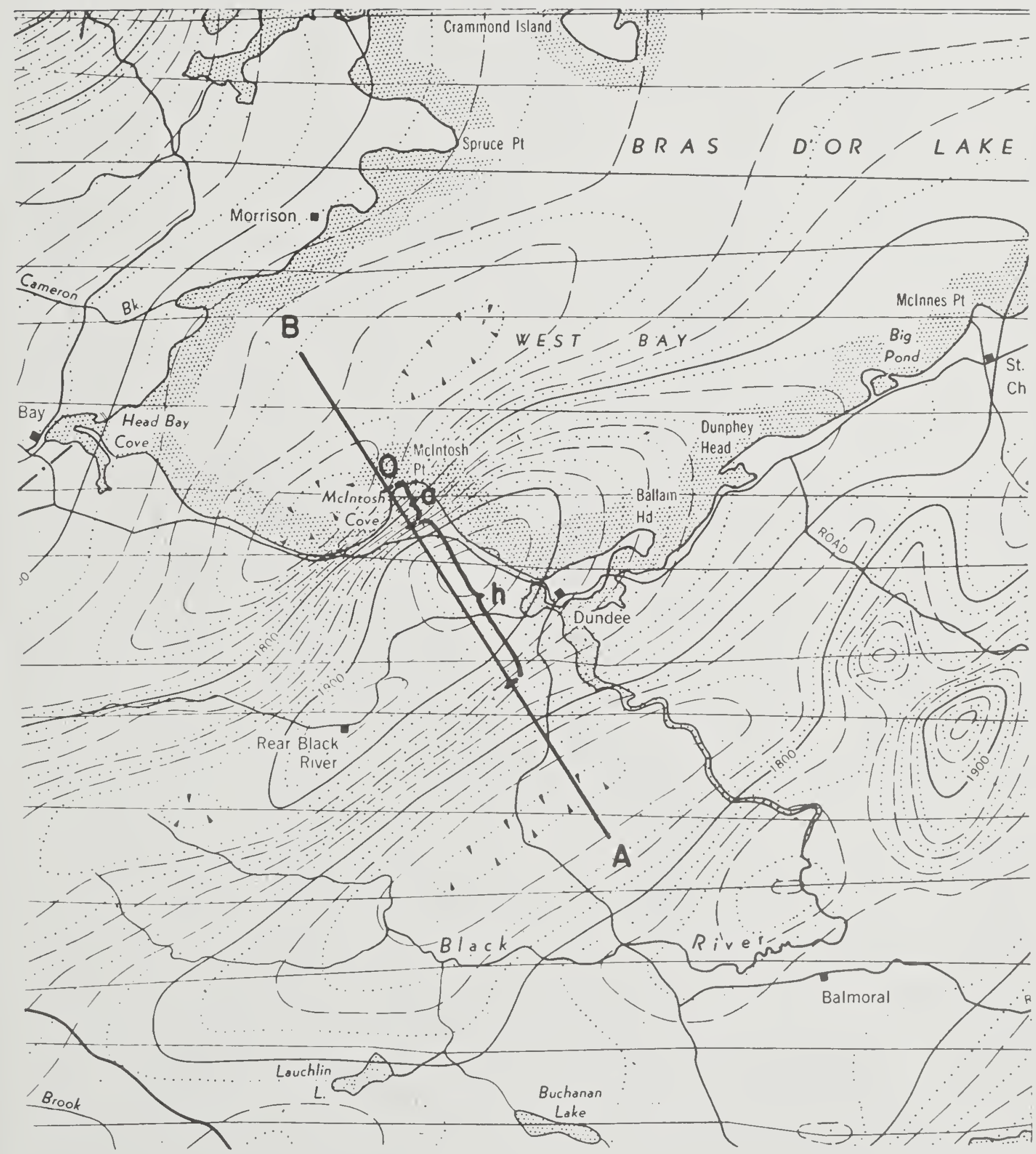

FIG. 4-4: PORT HAWKESBURY, N.S.

MAP $238 \mathrm{G}$ 
$4-20$

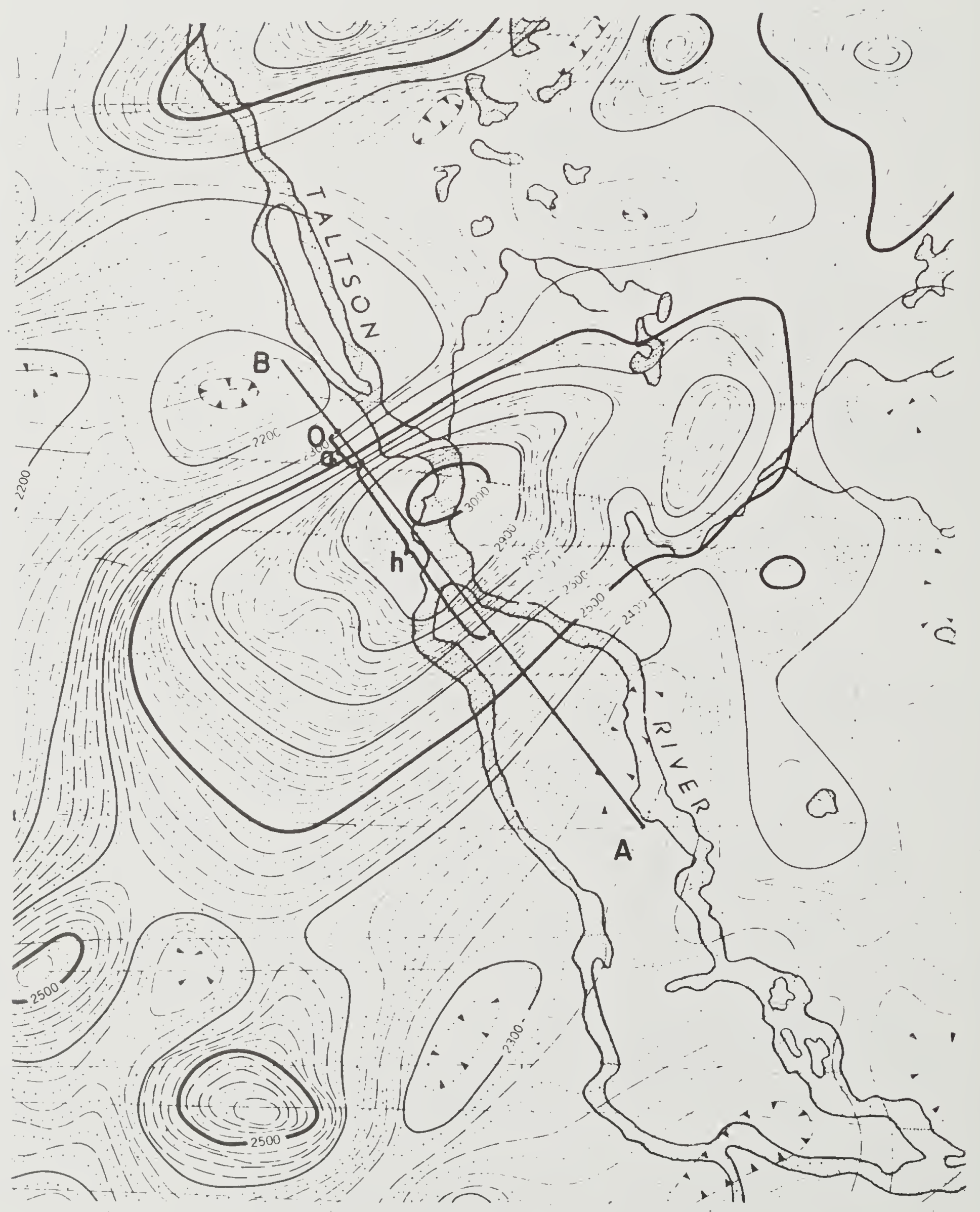

FIG. 4-5: DESKENATLATA LAKE SOUTH, NW TERRITORIES 


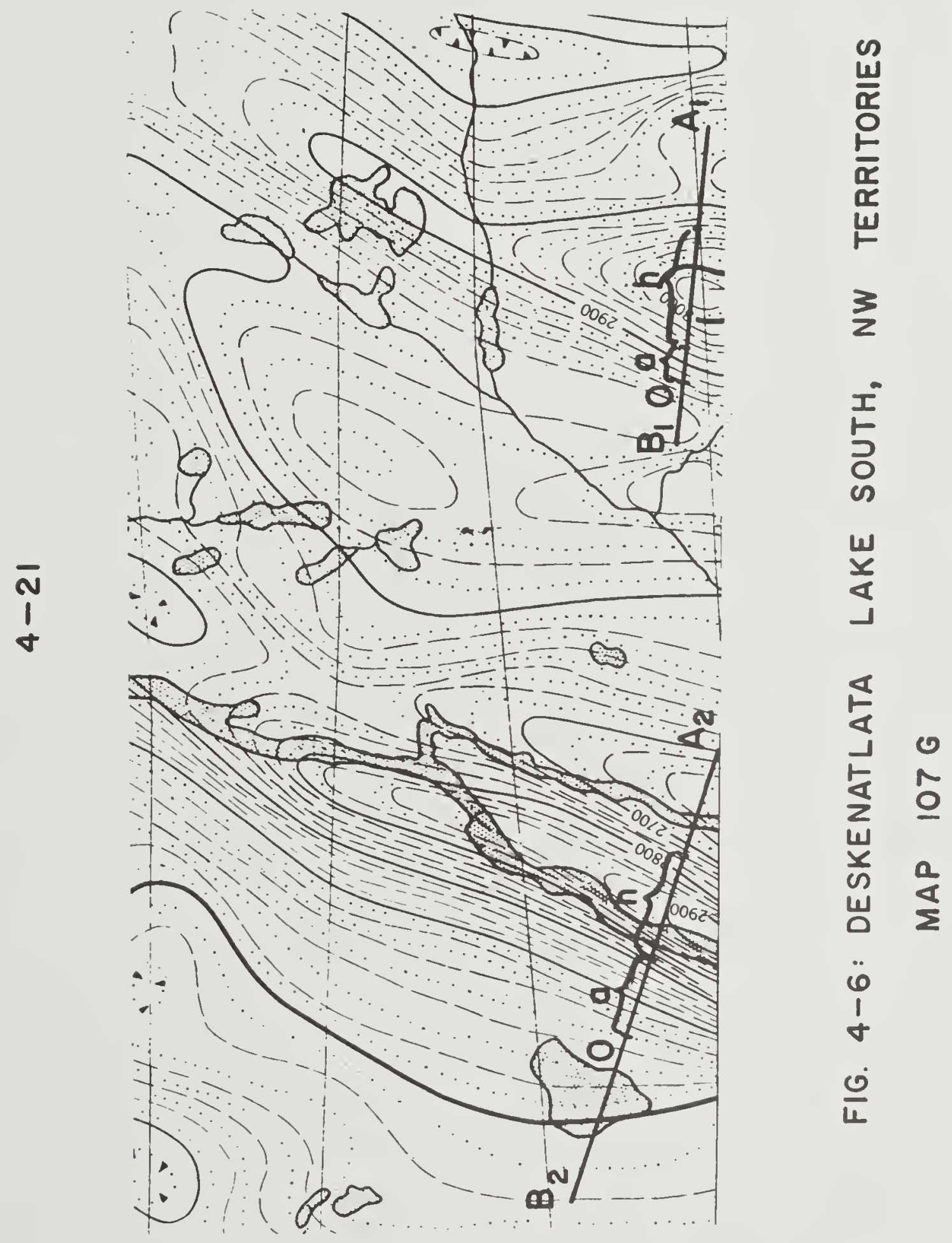




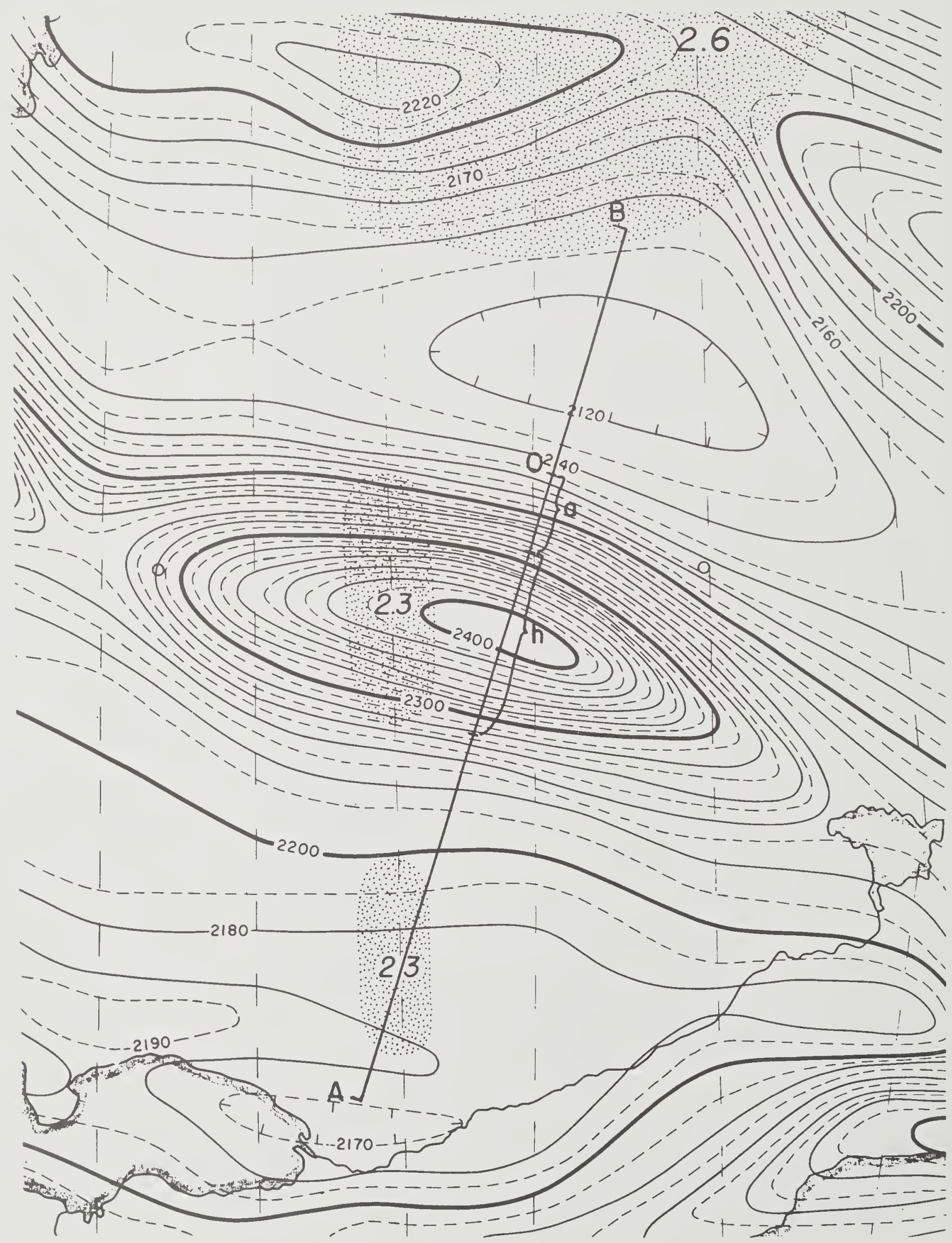




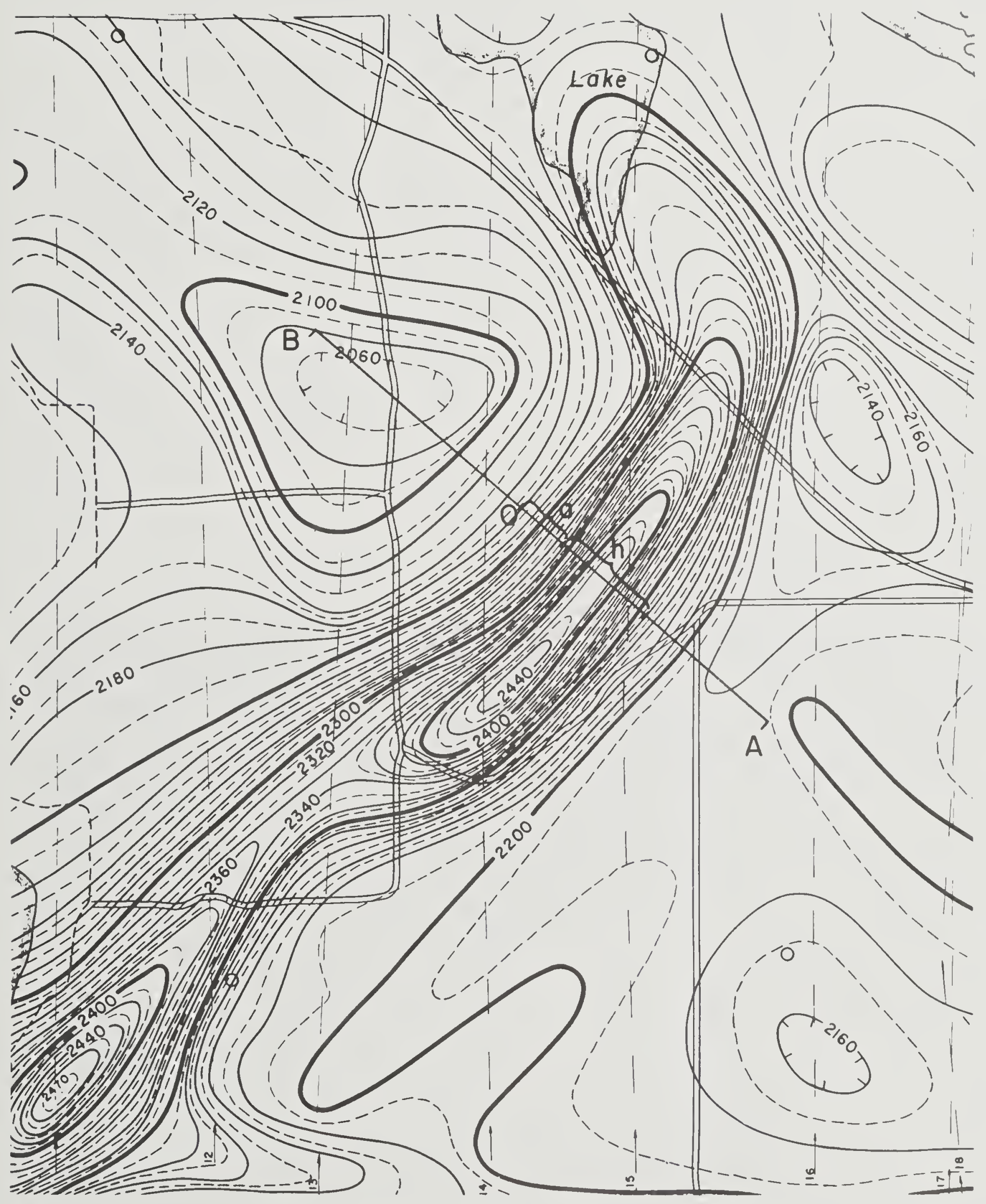

FIG. 4-8: GLADSTONE TOWNSHIP, ONTARIO 
$4-24$

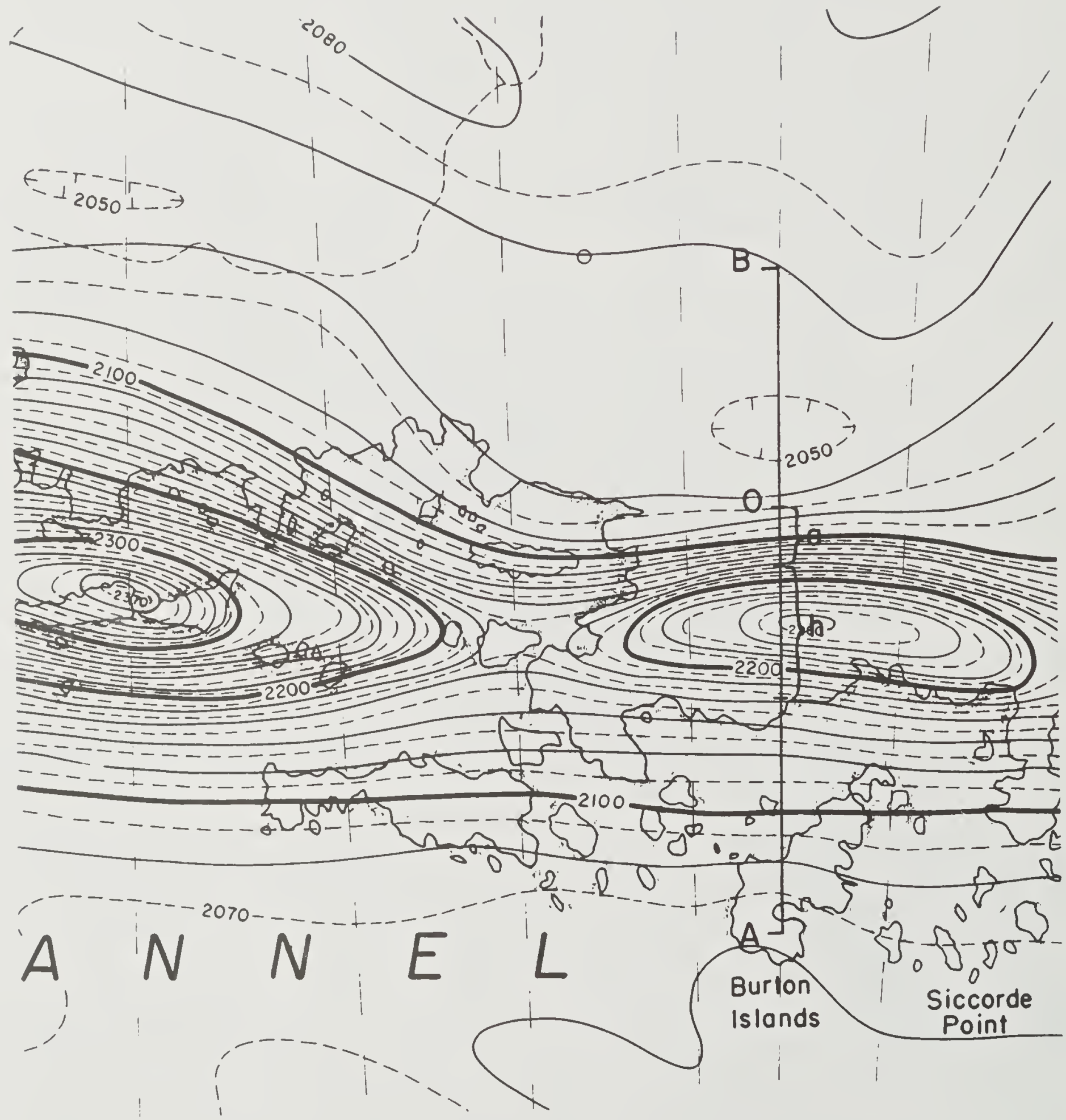

FIG. 4-9: BRIGHT TOWNSHIP, ONTARIO 


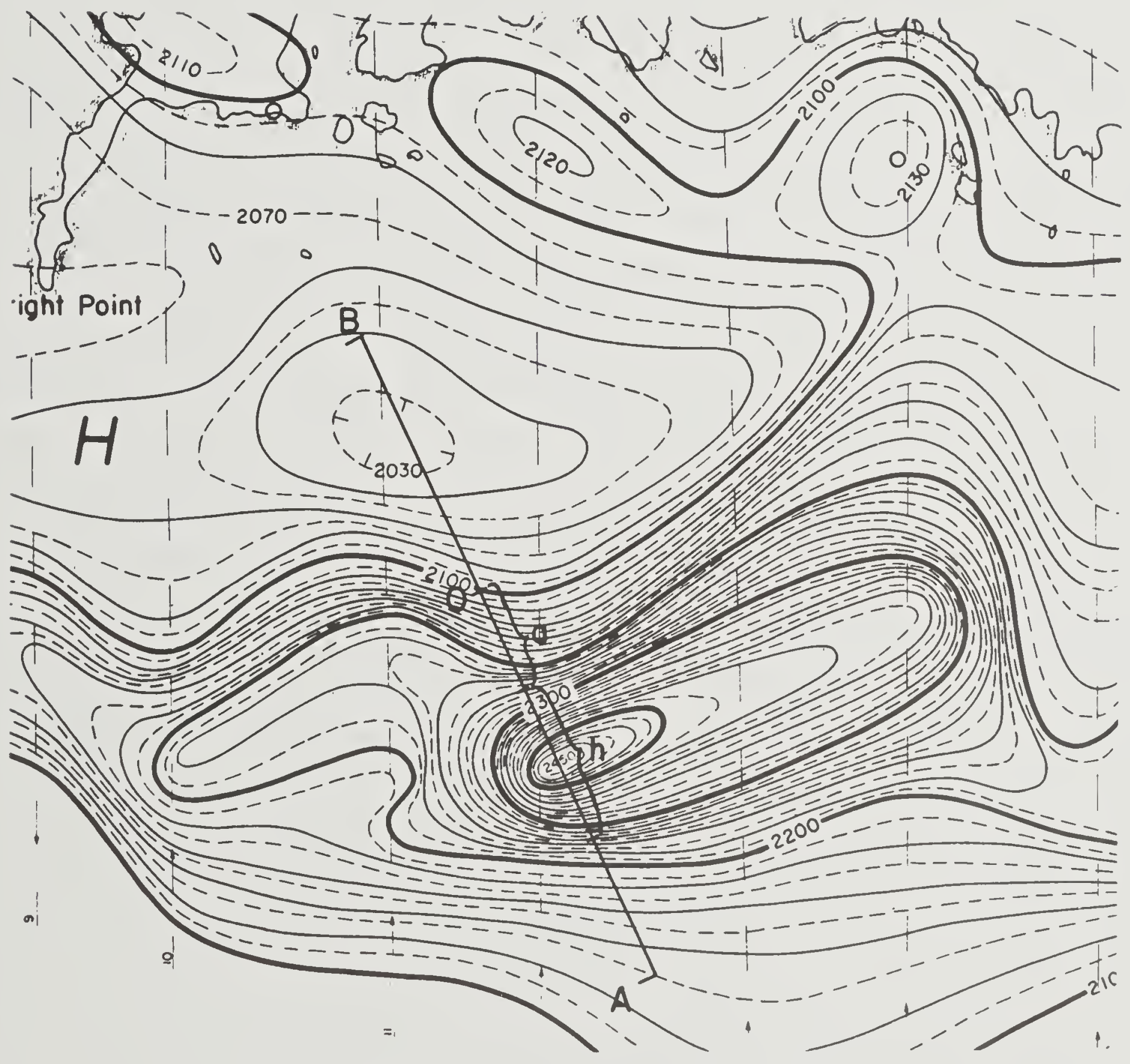

FIG. 4-IO: BRIGHT TOWNSHIP, ONTARIO 


\section{$4-26$}

E. Curves and Graphs

1. Computed curves

The bodies for which the anomaly curves have been computed are of the kind described in paragraph $B$ and the coordinate system used is essentially the same as shown in Fig. 4-1. As abscissa the ratio of the horizontal distance $x$ to the depth of burial d for each curve has been plotted in order to facilitate the graphical representation. The vertical extent $D$ is the same for all bodies, namely $15 \mathrm{~km}$. The depth of burial d in $\mathrm{km}$ is indicated on each curve. In all cases the assumed total intensity $F$ of the undisturbed field is 50,000 gammas and the susceptibility $\mathrm{k}$ is $10^{-3} \mathrm{emu}$.

For width to depth ratios smaller than one the curves practically do not change their character except that their amplitude is multiplied by a factor proportional to $\mathrm{W} / \mathrm{d}$. That is the curves for $\mathrm{W} / \mathrm{d}=1 / 2 \mathrm{e} . \mathrm{g}$. are approximately the same as the curves for $w / d=I$ multiplied by the factor of 1/2. Therefore, onIy curves for $w / d \geqslant I$ are shown here. 
FIGURE 4-II

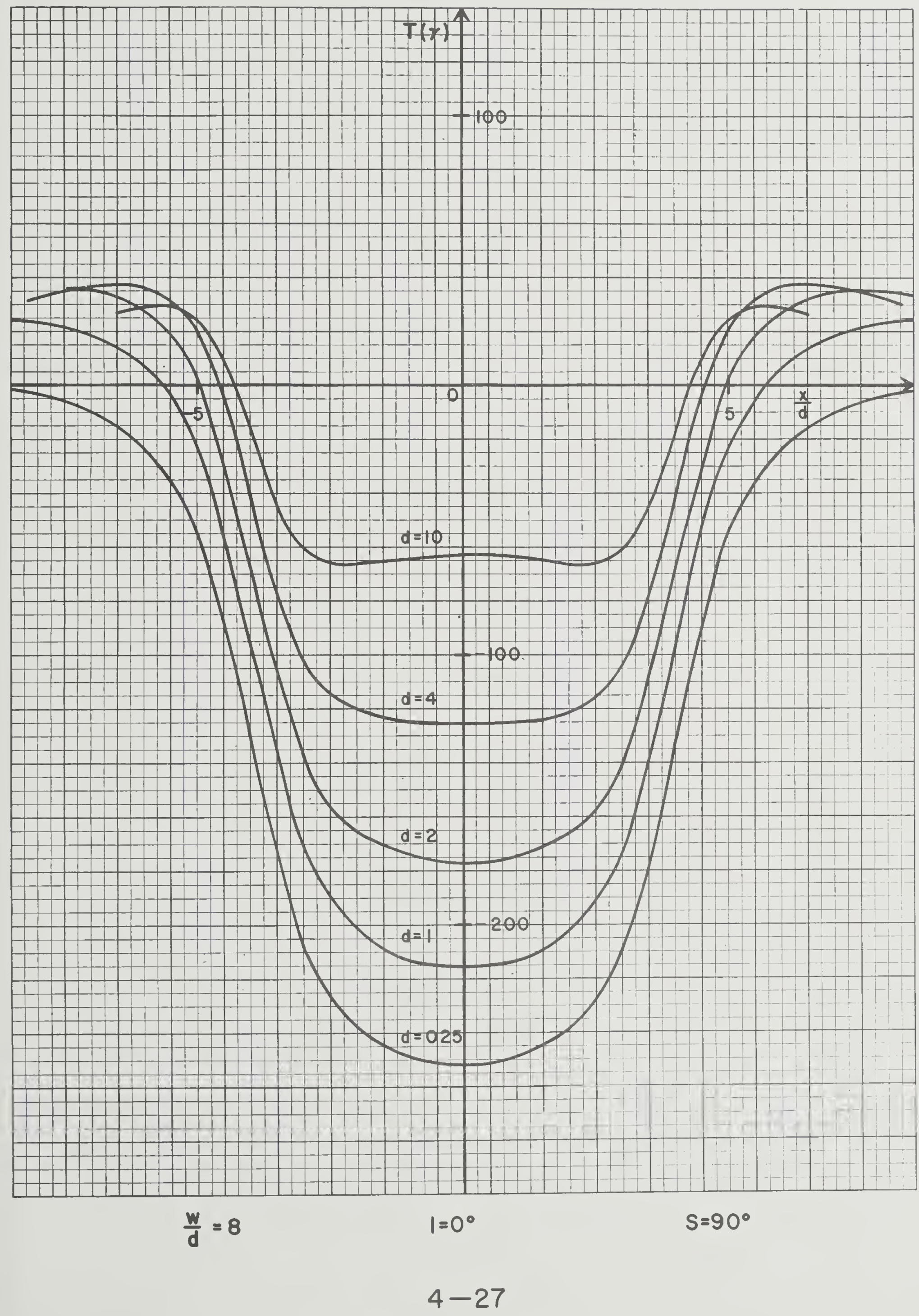


FIGURE $\quad 4-12$

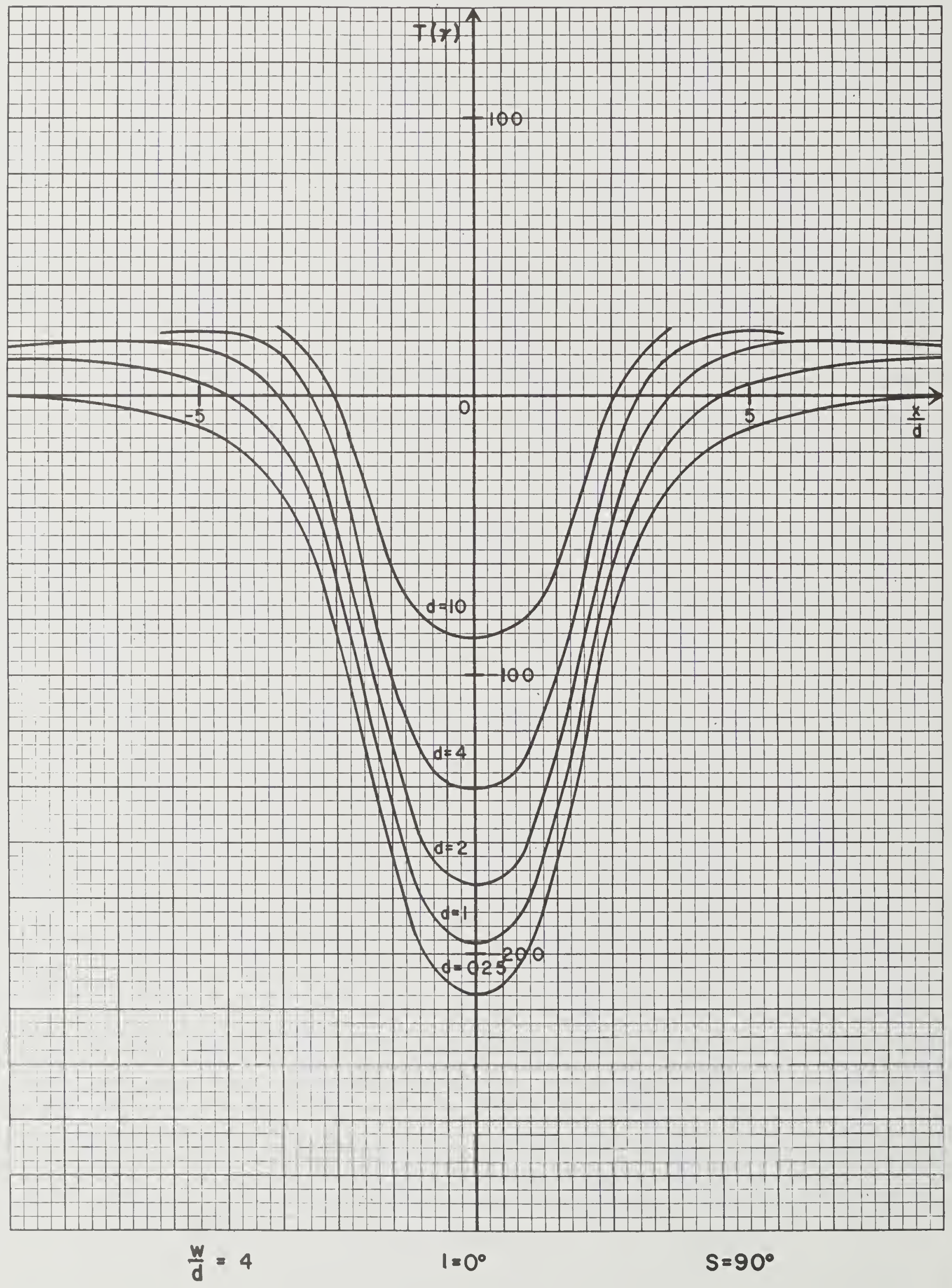


FIGURE $4-13$

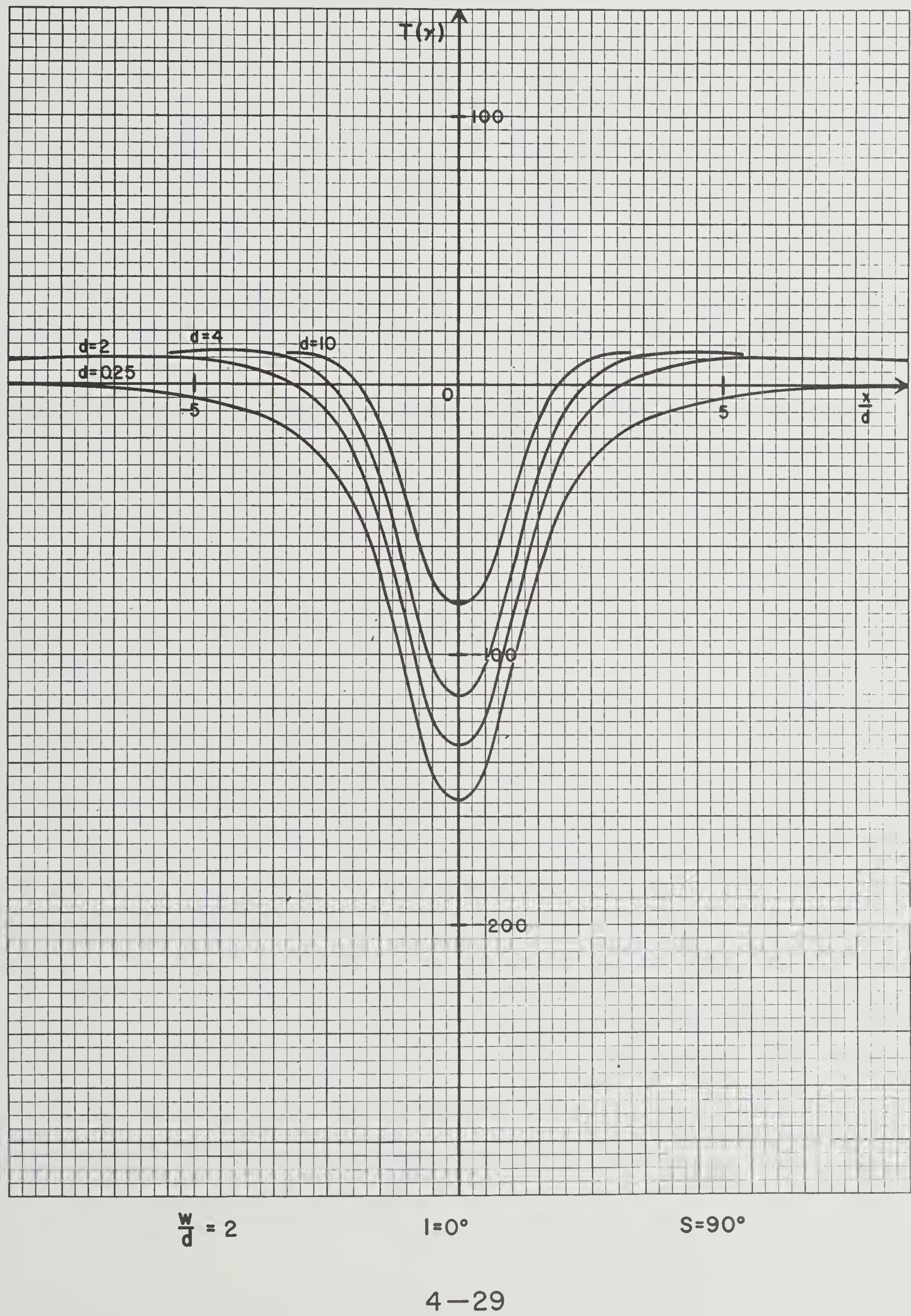


FIGURE 4-14

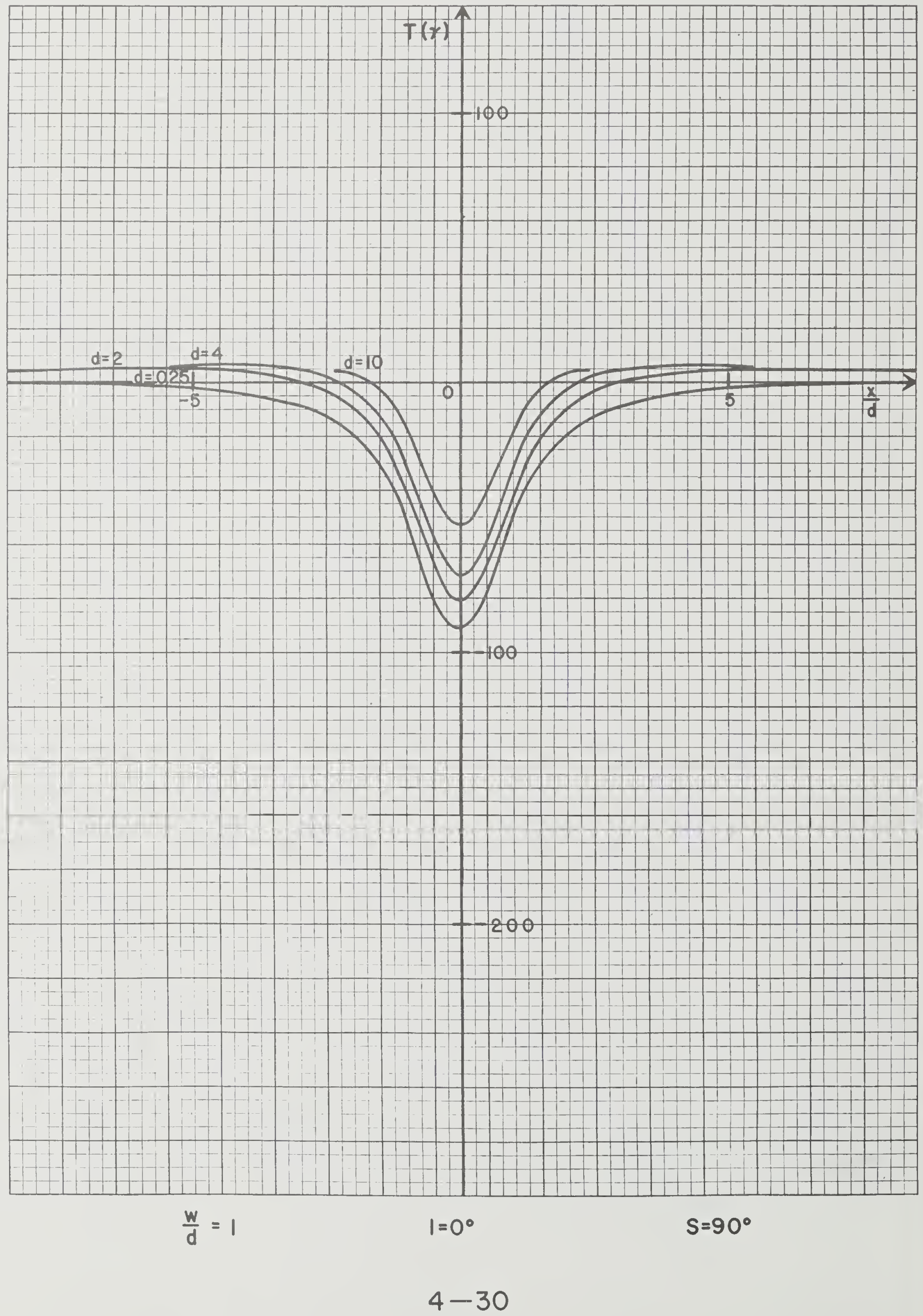


FIGURE $\quad 4-15$

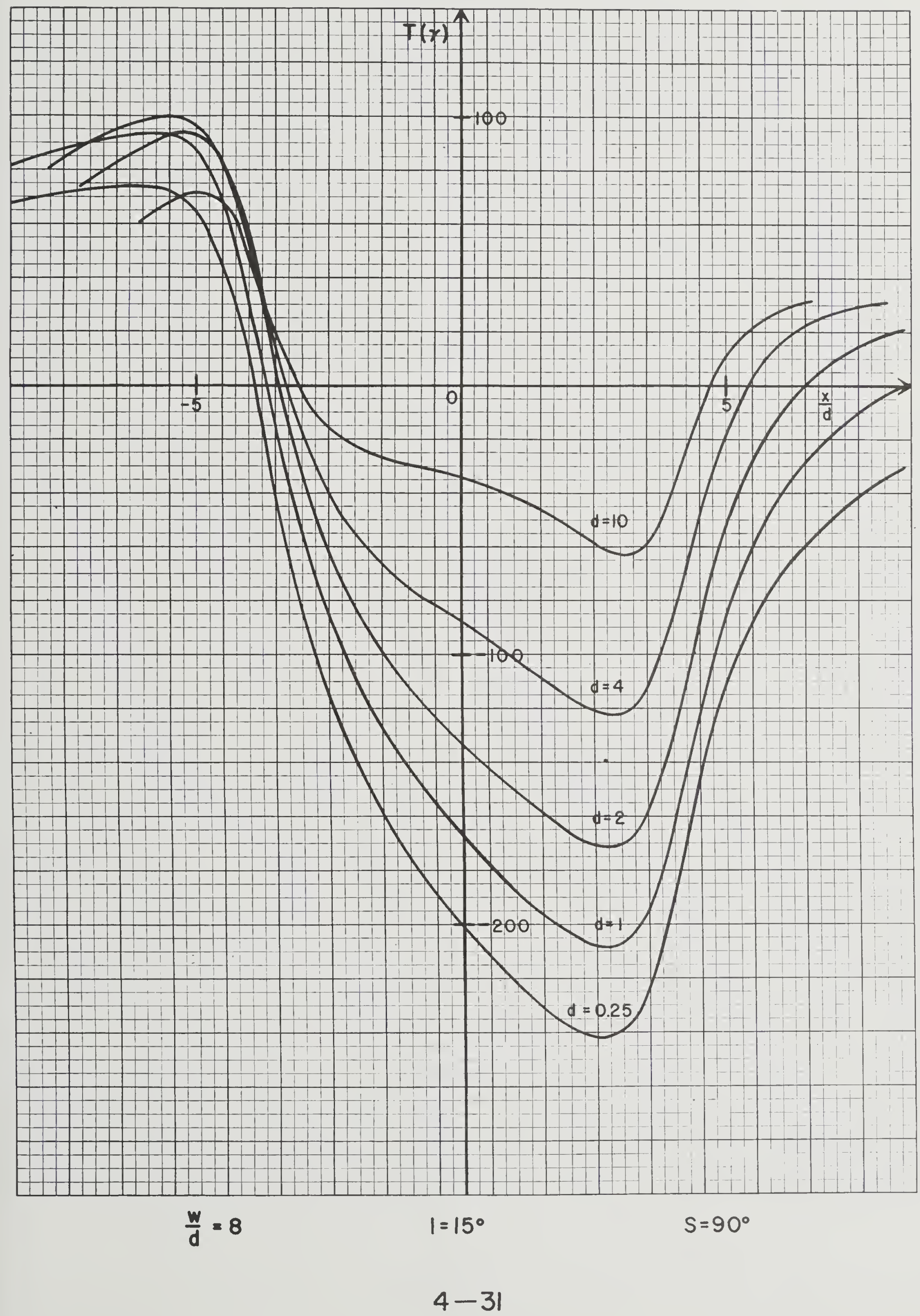


FIGURE $\quad 4-16$

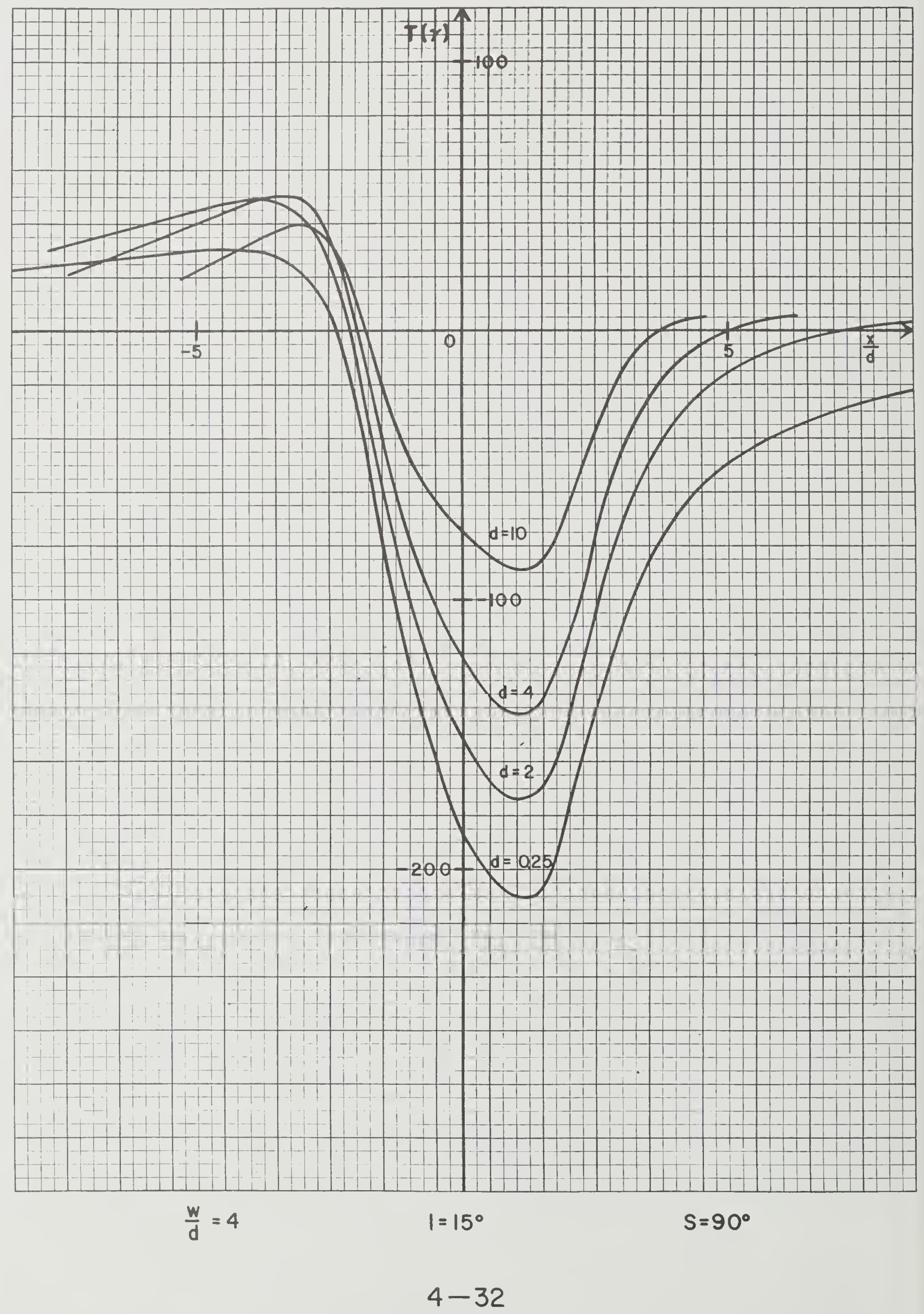


FIGURE $\quad 4-17$

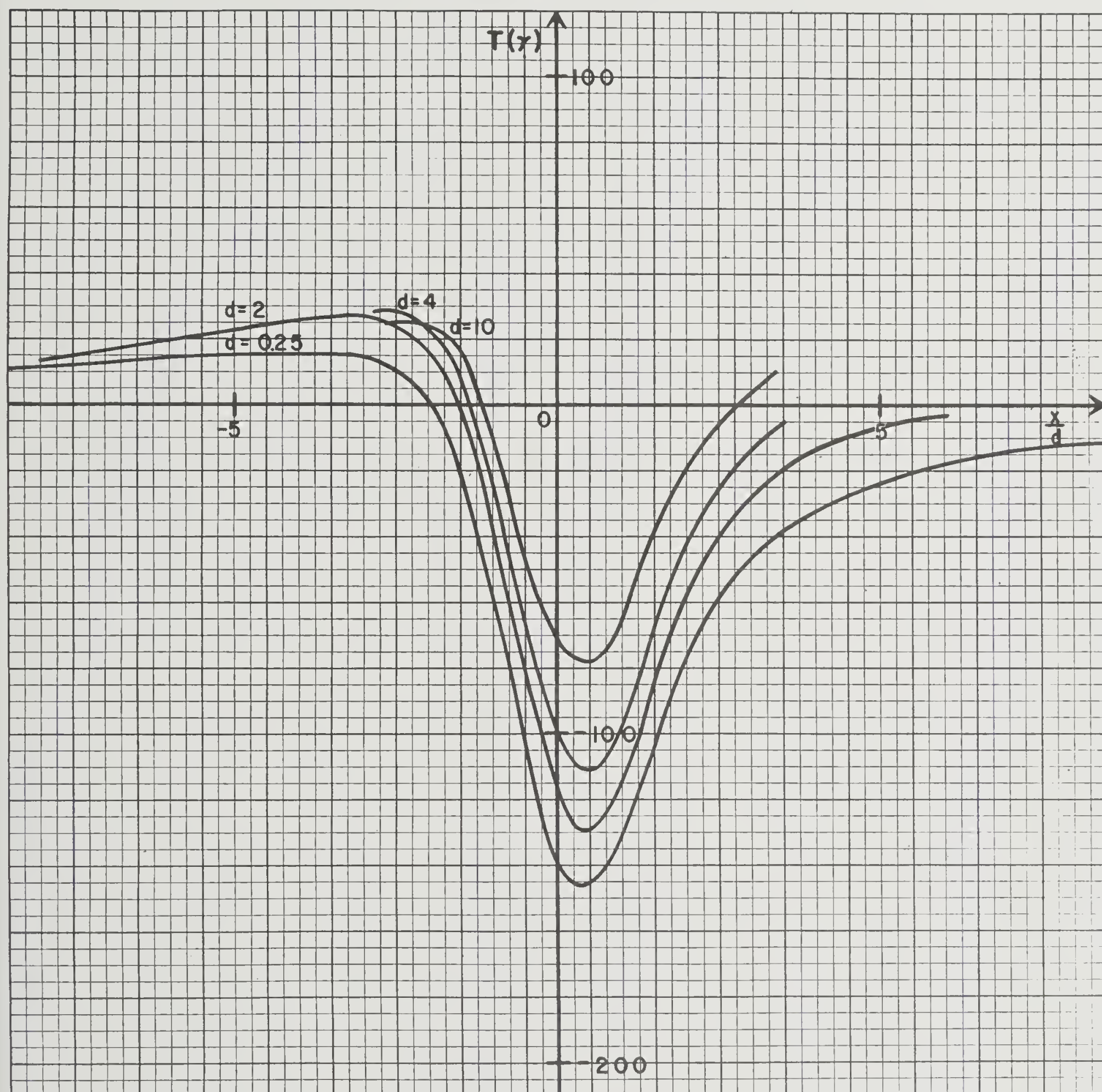

\begin{tabular}{|l|l|l|l|l}
\hline &
\end{tabular}

$$
\begin{aligned}
& \begin{array}{lll}
\frac{w}{d}=2 & \quad l=15^{\circ} & S=90^{\circ}
\end{array} \\
& 4-33
\end{aligned}
$$


FIGURE $\quad 4-18$

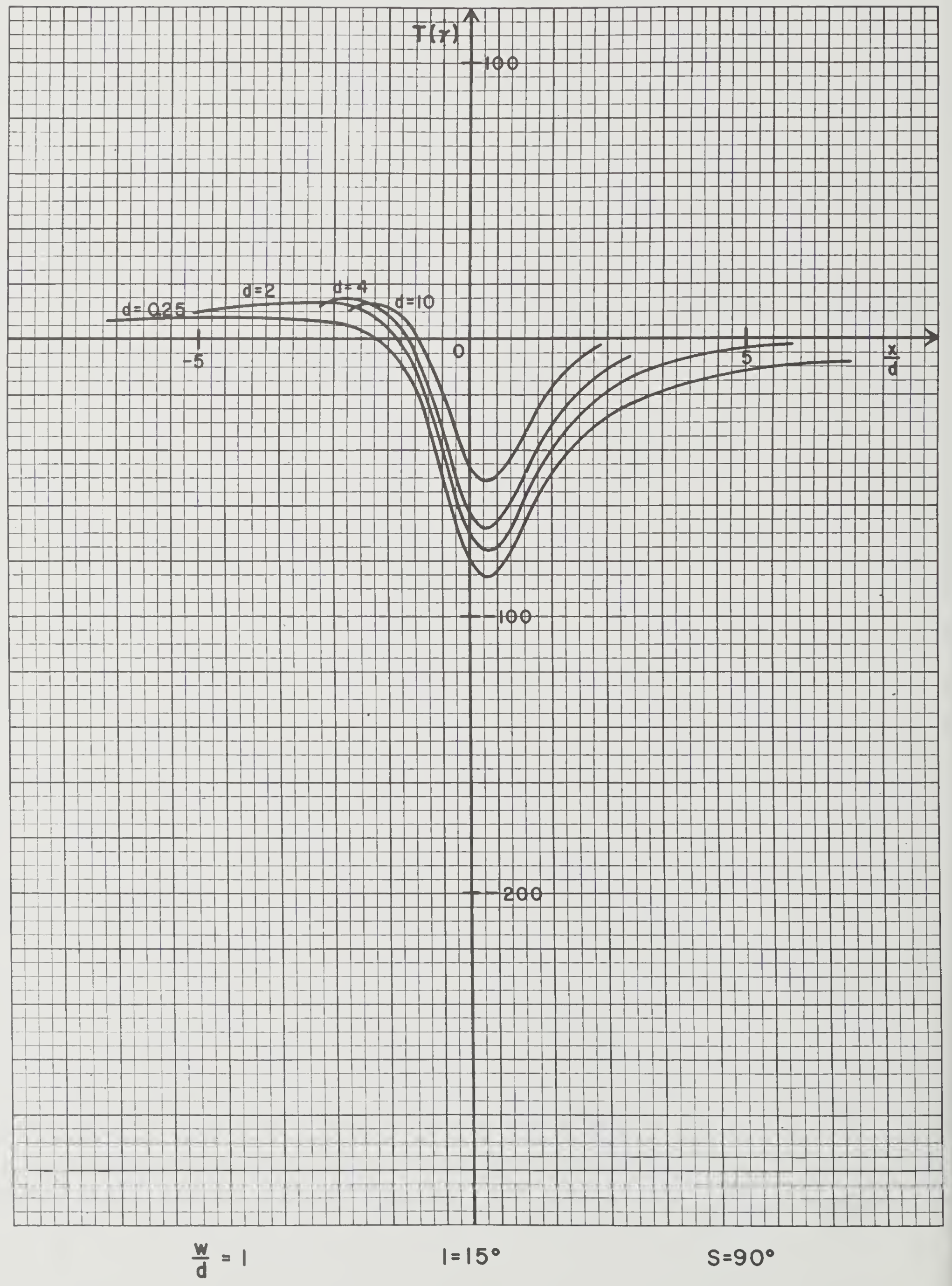


FIGURE $\quad 4-19$

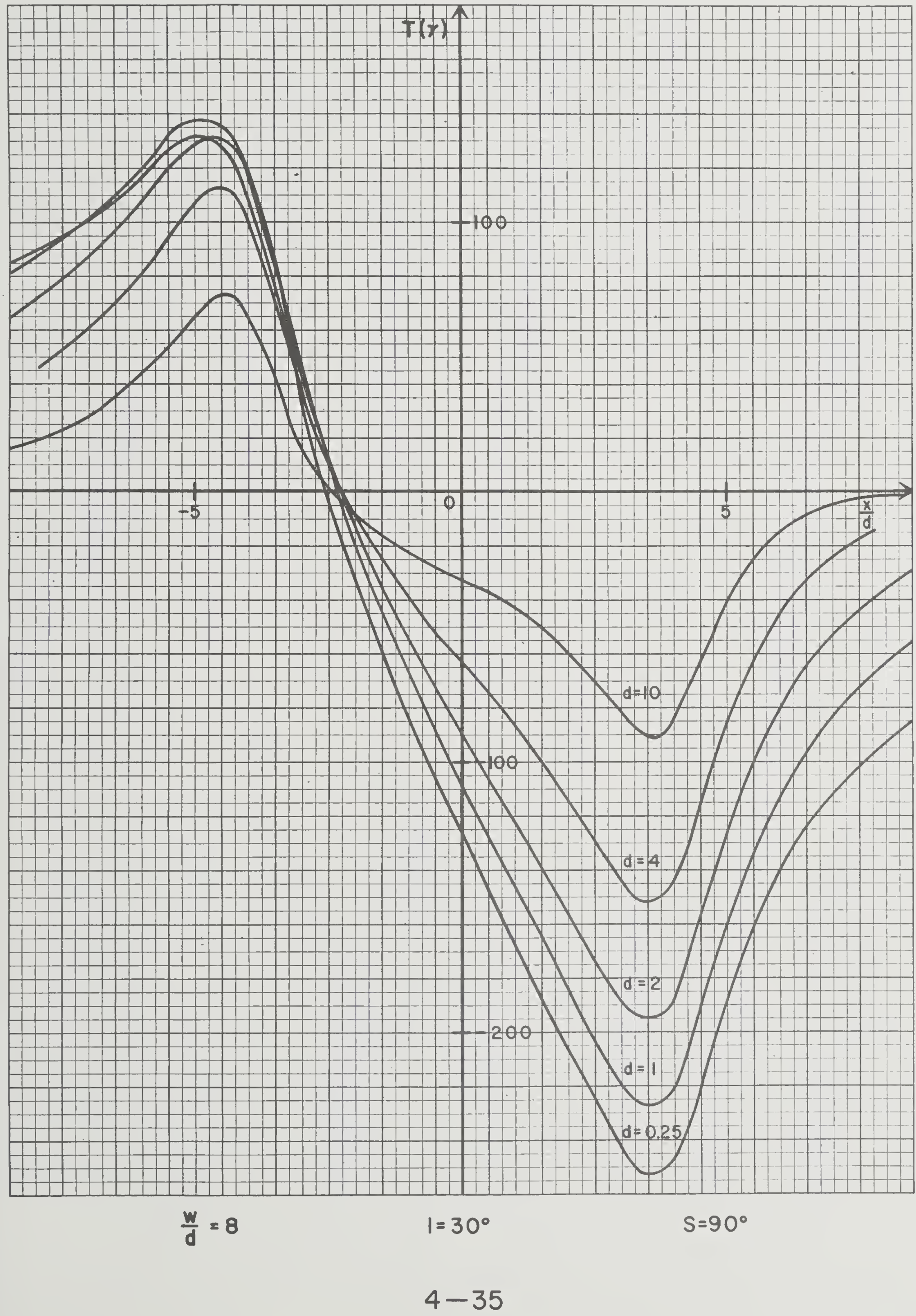


FIGURE 4-20

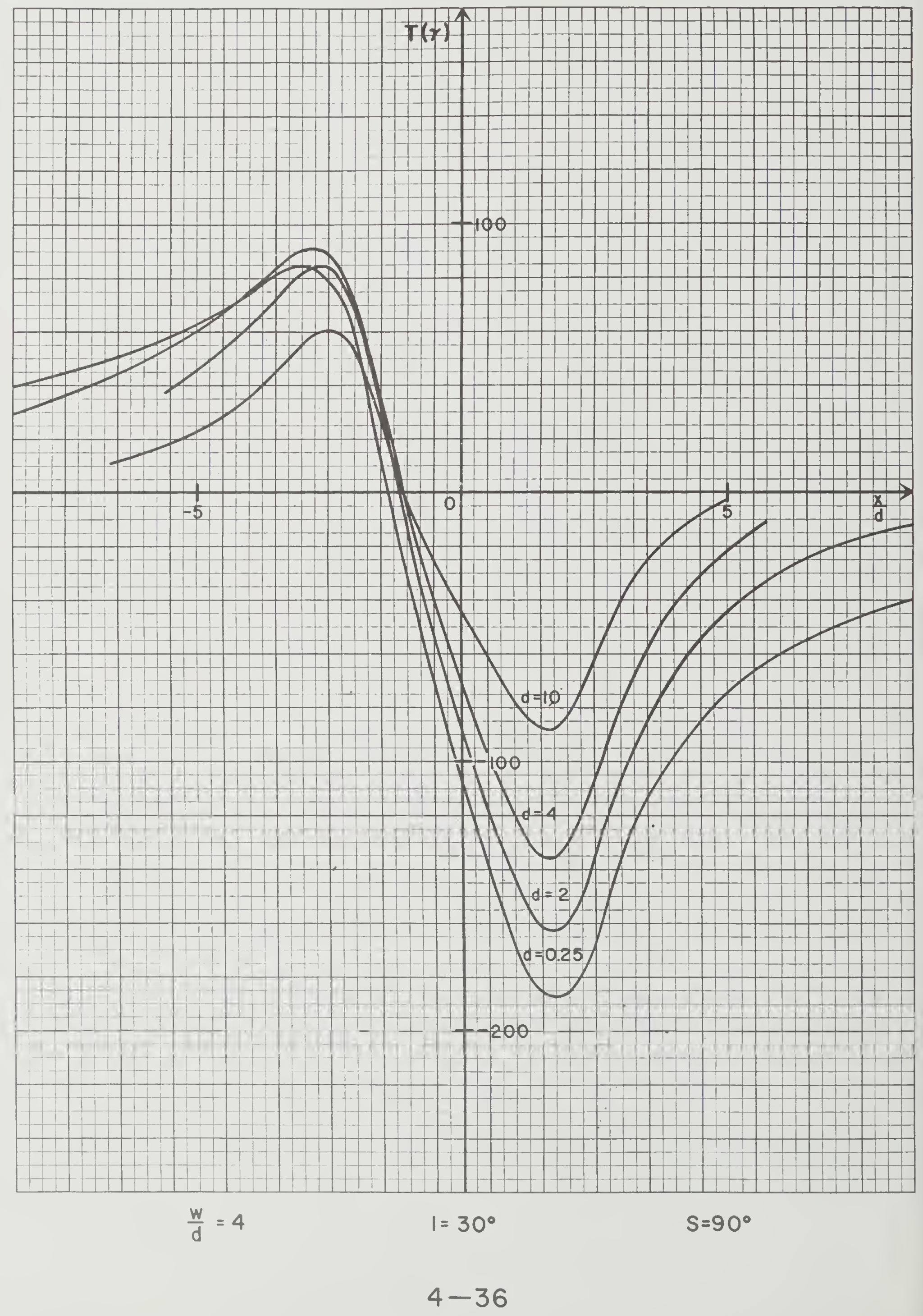


FIGURE 4-2I

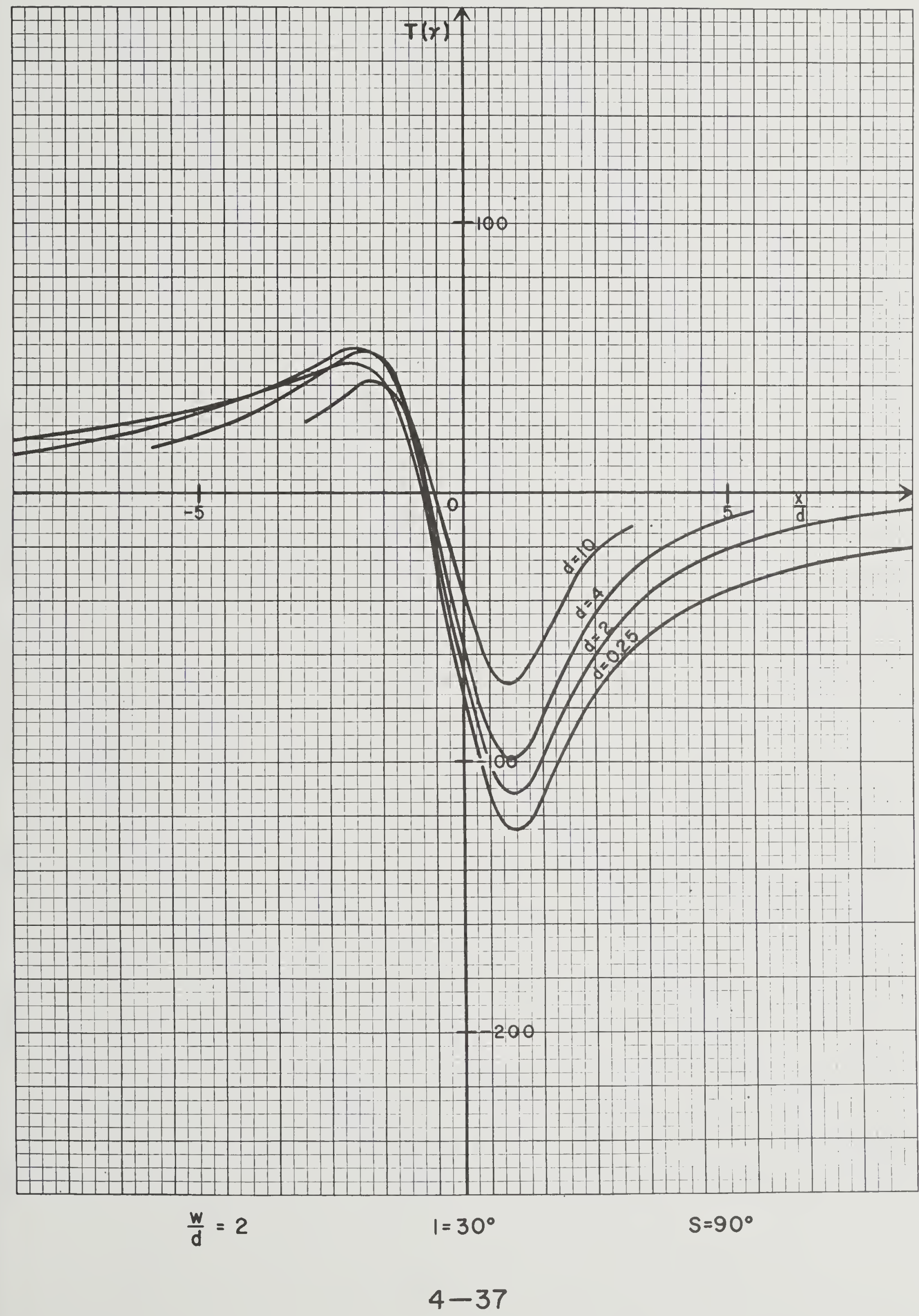


FIGURE $\quad 4-22$

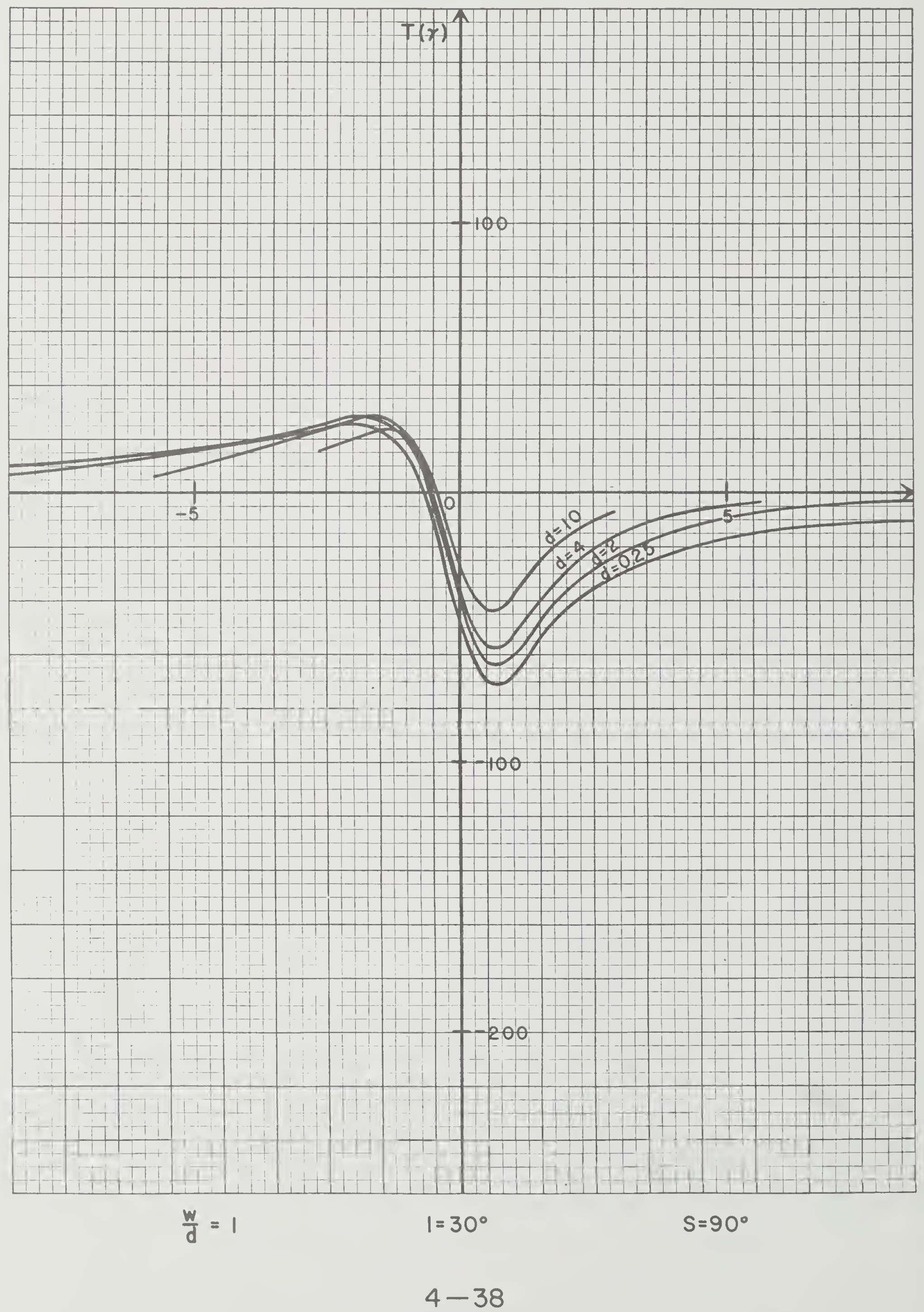


FIGURE $\quad 4-23$

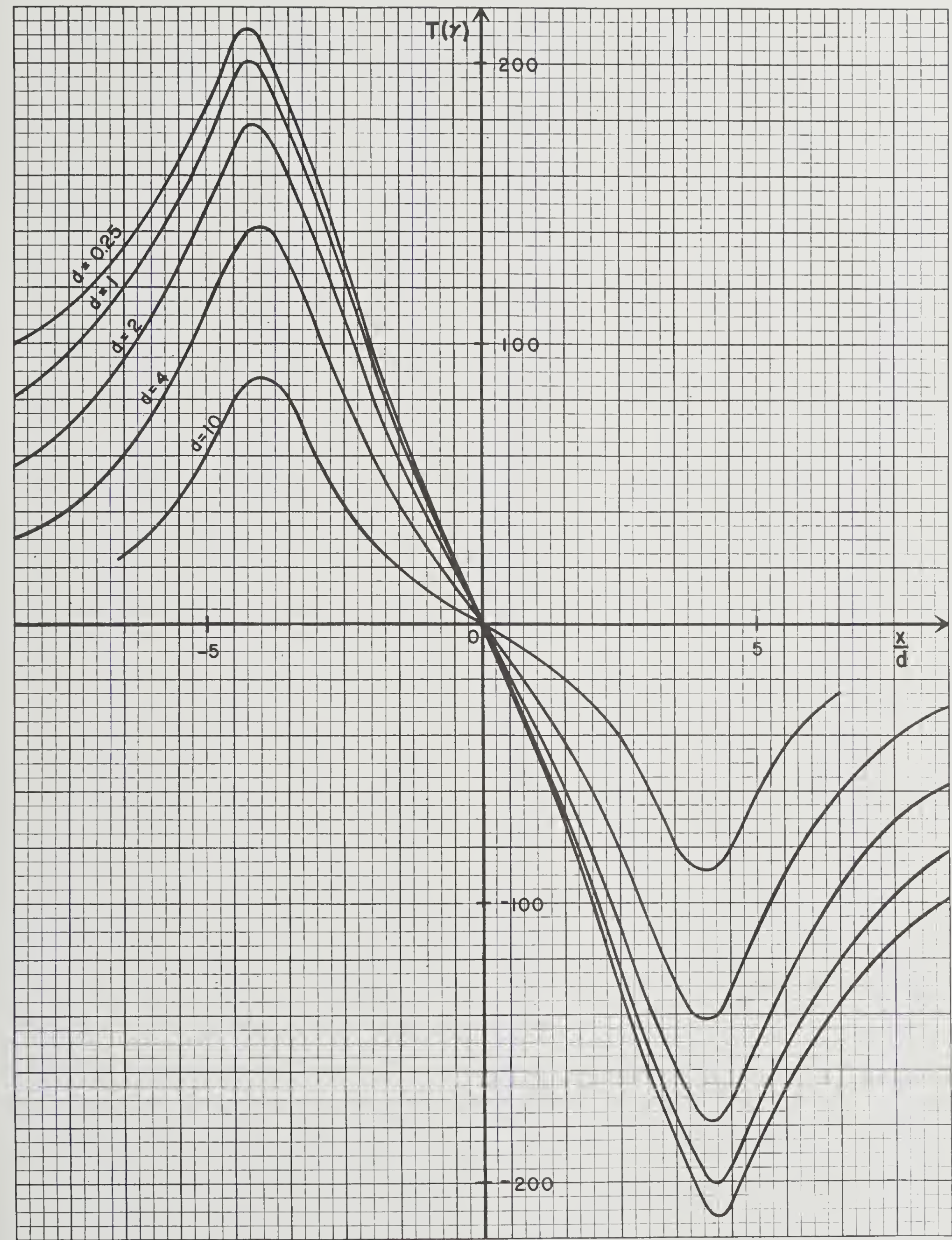

$$
\frac{w}{d}=8 \quad I=45^{\circ} \quad S=90^{\circ}
$$


FIGURE 4-24

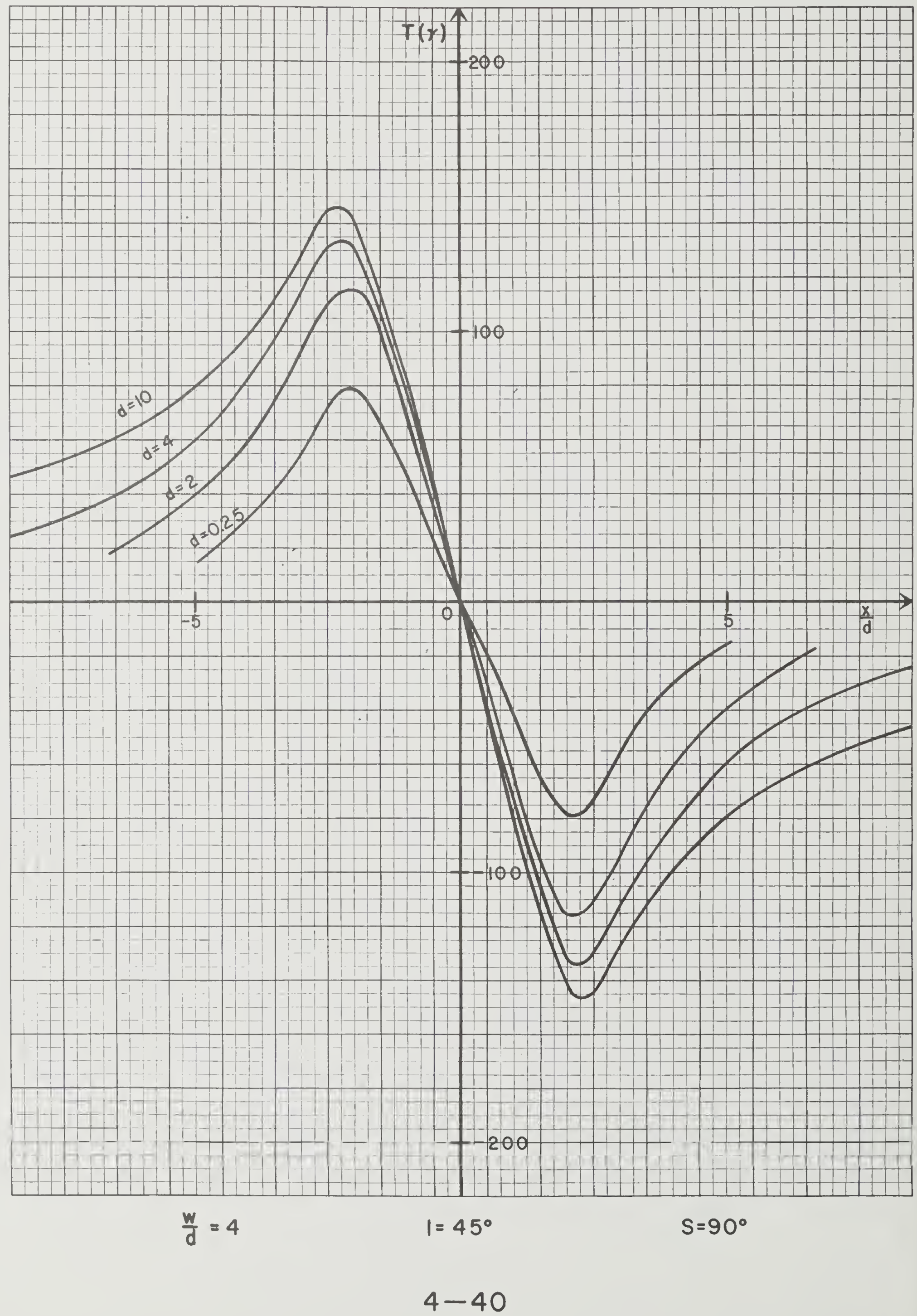


FIGURE $\quad 4-25$

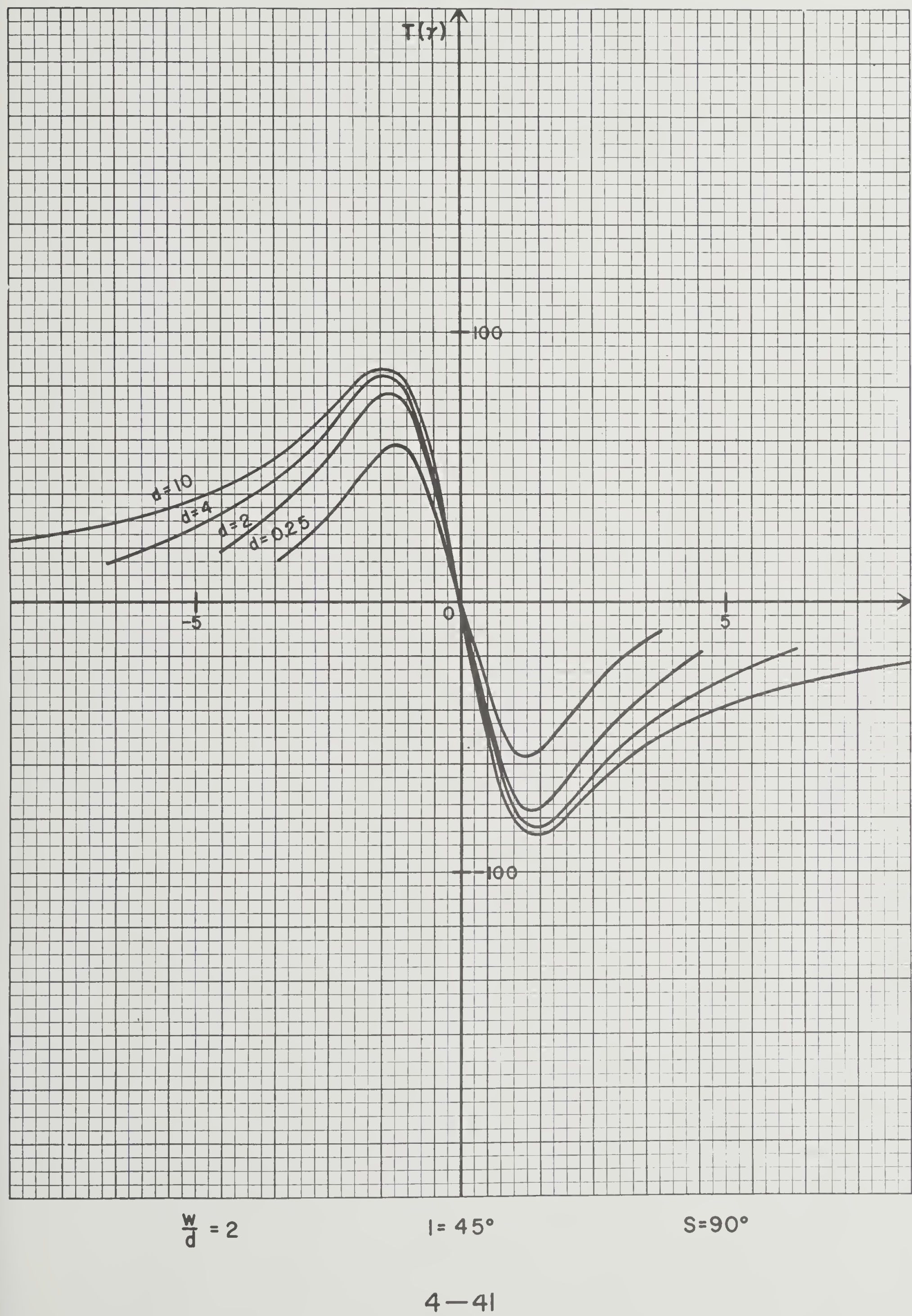


FIGURE 4-26

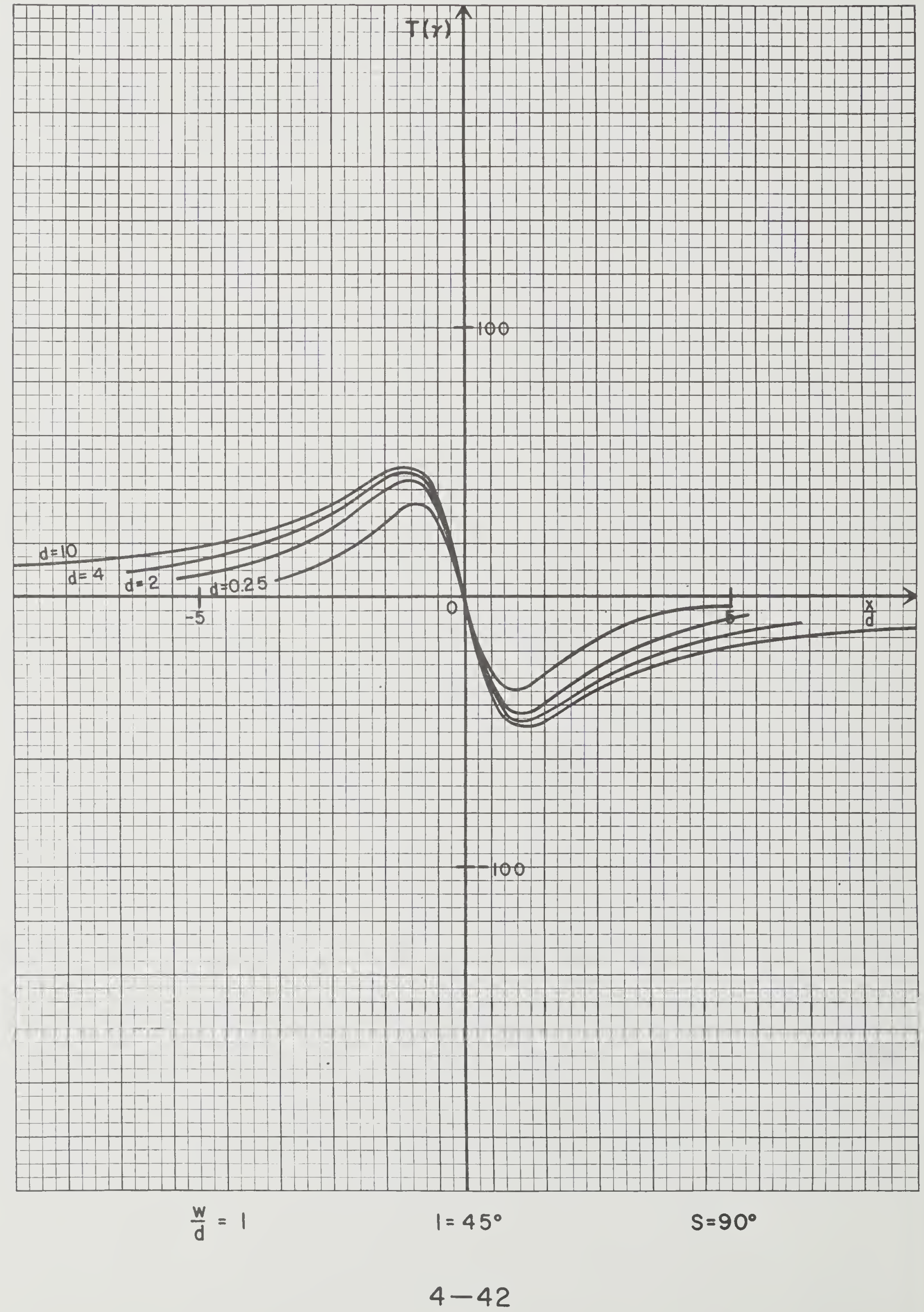




\section{Nomograms and Tables}

The values of the tables and the nomograms have been obtained by measuring the corresponding distances on the computed curves.

\section{a. Nomograms}

Nomogram A for each inclination shows two families of curves. Both of them give the half-width $h$ as a function of the ratio b. For the solid curves the w/d ratio is held constant, while the dashed curves are plotted for constant depths of burial.

On nomogram $B$ the half-width is plotted as a function of depth for constant $W / d$ ratios.

b. Tables

As equations (4-1) and (4-2) show, the strike influences anomaly curves for different body parameters in a different way. Corrections for the strike for different values of depth and width are given in tables $4-1$ to $4-4 . \Delta h$ and $\Delta b$ are the percentages that have to be added to the measured values of $h$ and $b$ in order to obtain the values which would have been measured for a strike of $90^{\circ}$.

For inclinations of less than $45^{\circ}$ and strikes near $0^{\circ}$ the positive part of the anomalies becomes larger than the negative one, and in these cases it is more convenient to measure the distances $\bar{h}$ and $\bar{a}$ from the maximum instead of using the minimum (see figure 4-27). 
$4-44$

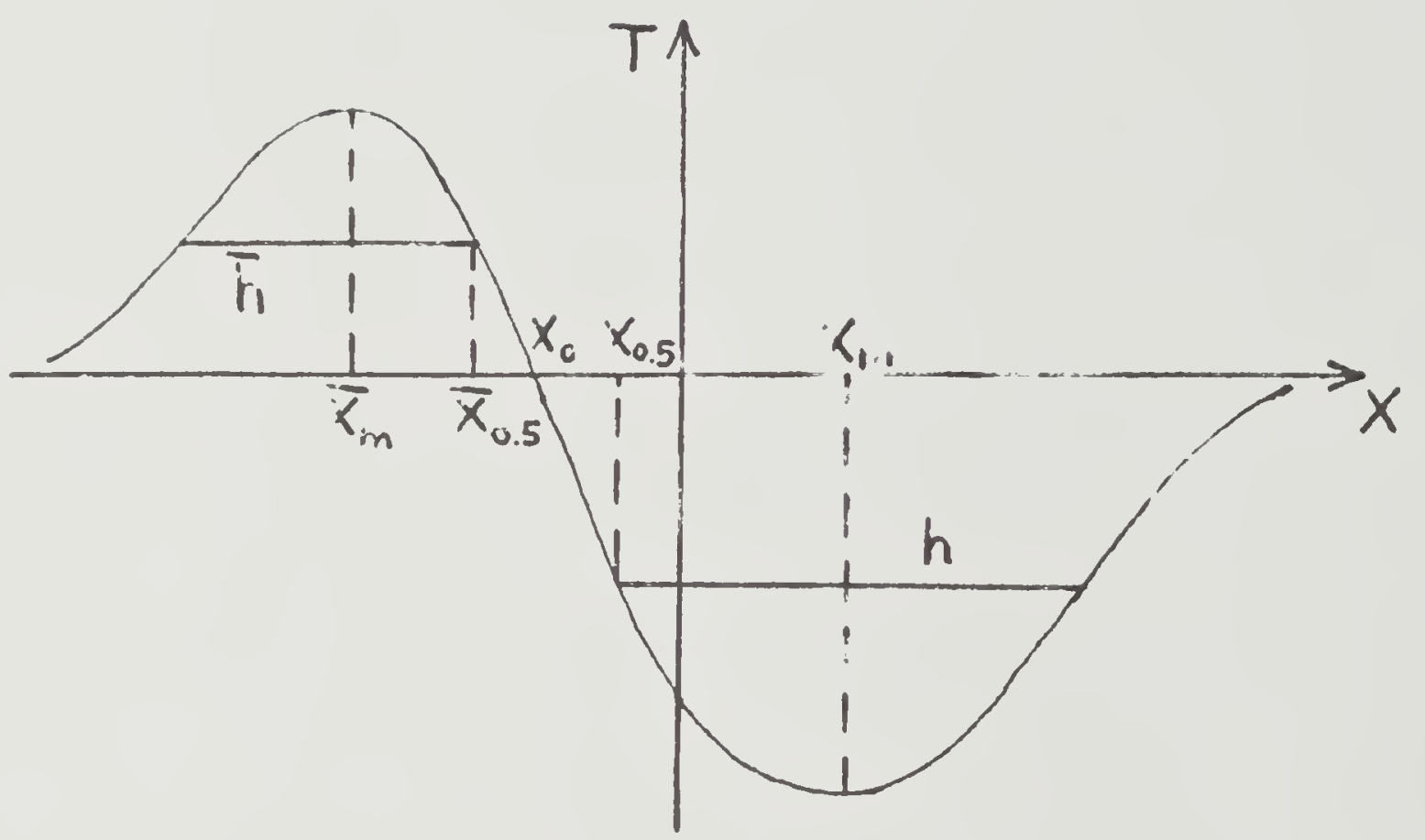

Fig. 4-27 


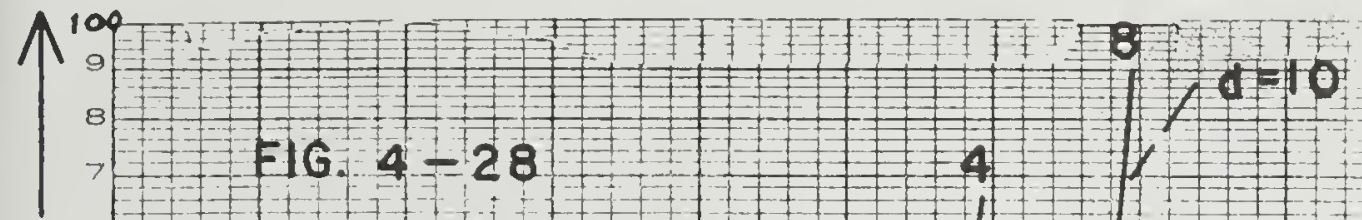

NOMOGRAM A

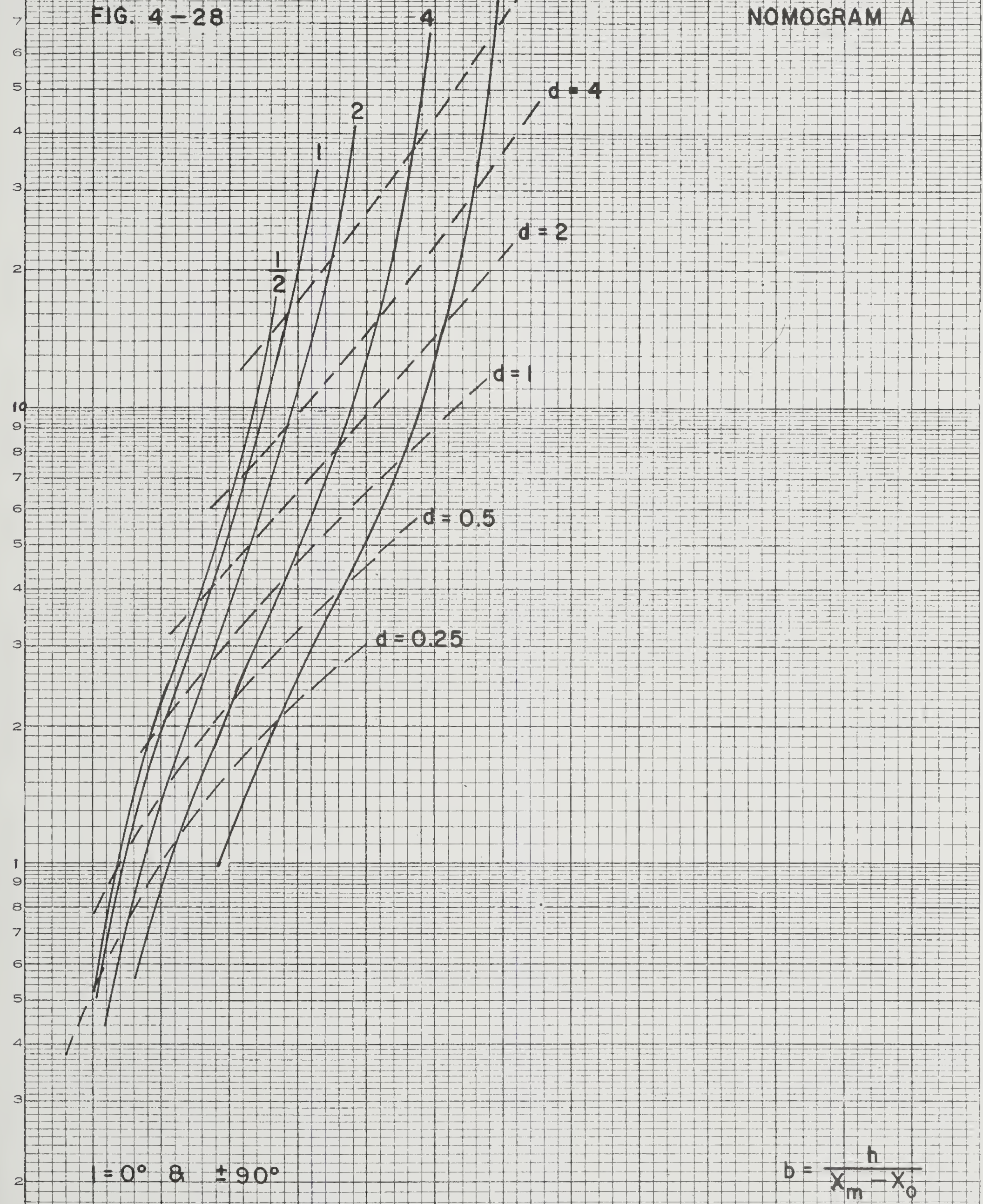

.

$$
b=\frac{h}{x_{m}-x_{0}}
$$


$716: 4-29$
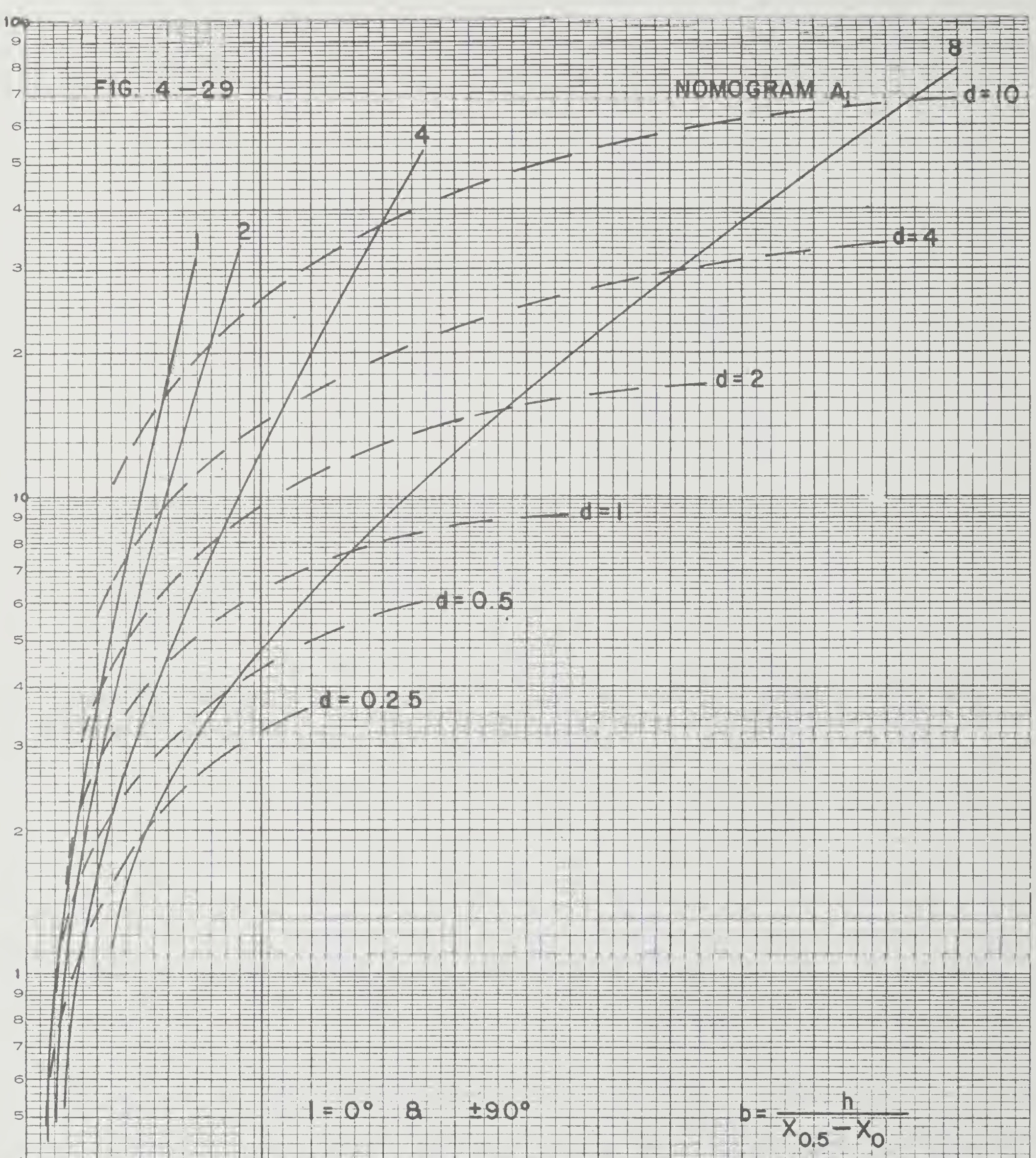

\footnotetext{
$\frac{\begin{array}{c}E N L A R G D \\ \text { SECTHON }\end{array}}{\text { SEE }}$

NOMOGRAM A2
} 


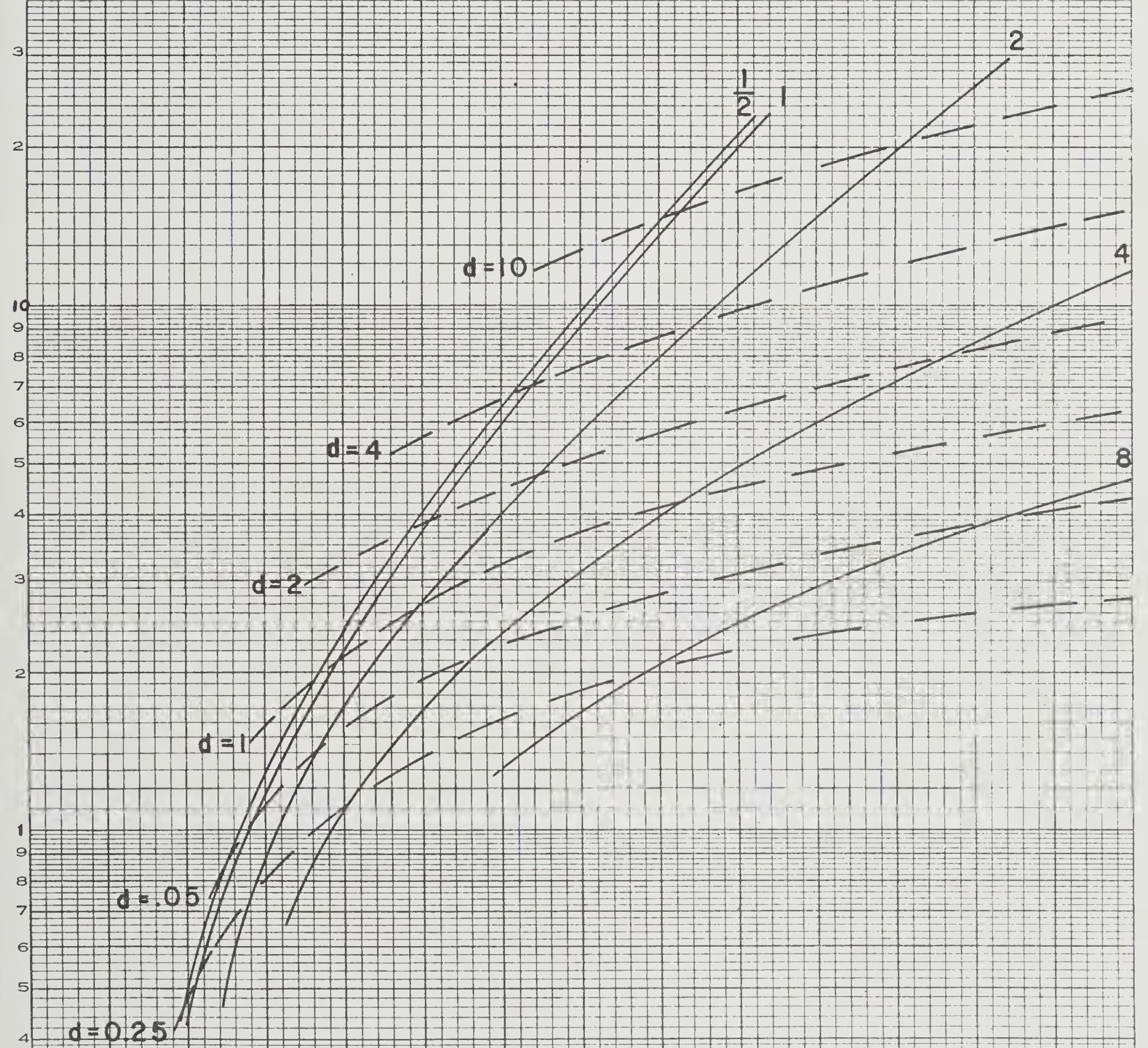

$d=0.25$

(1)

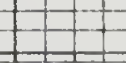

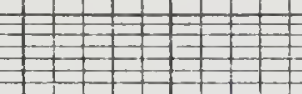

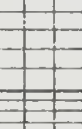

\section{$+$}

$+1$

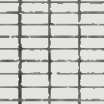

$+$

NOMOGRAM $A_{2}$ 


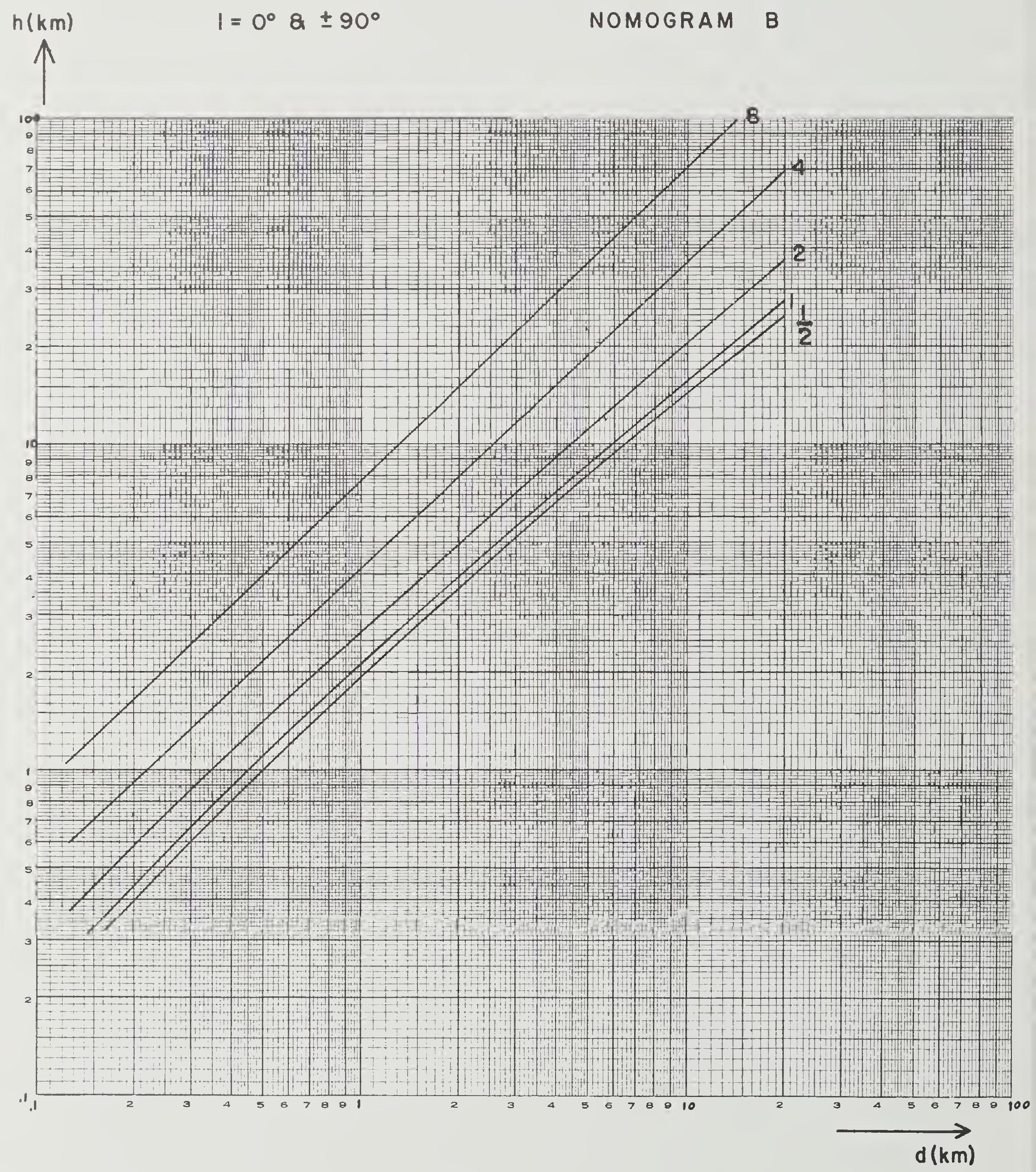

F|G. 4-3| 


\section{$h(k m)$}

10

1

FIG $-4-32$

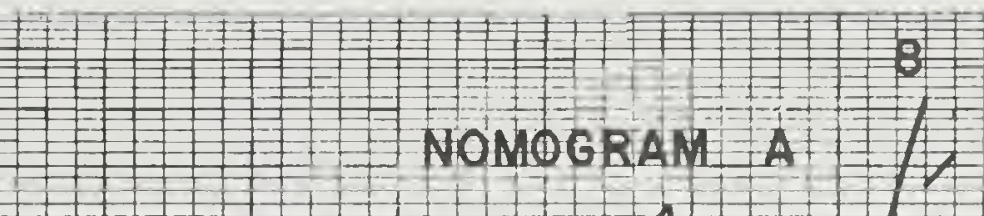

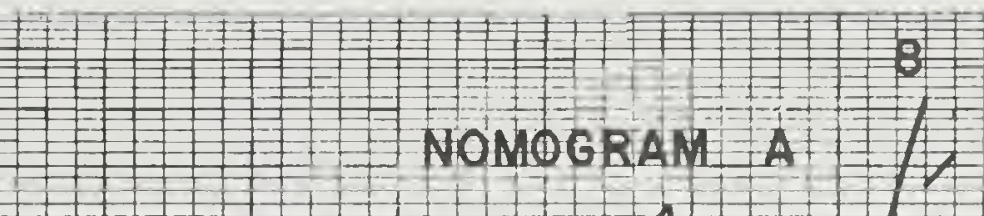

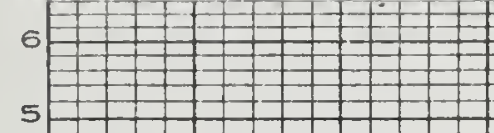

1

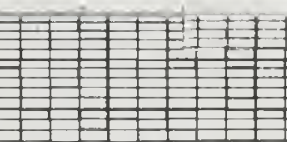

$+$
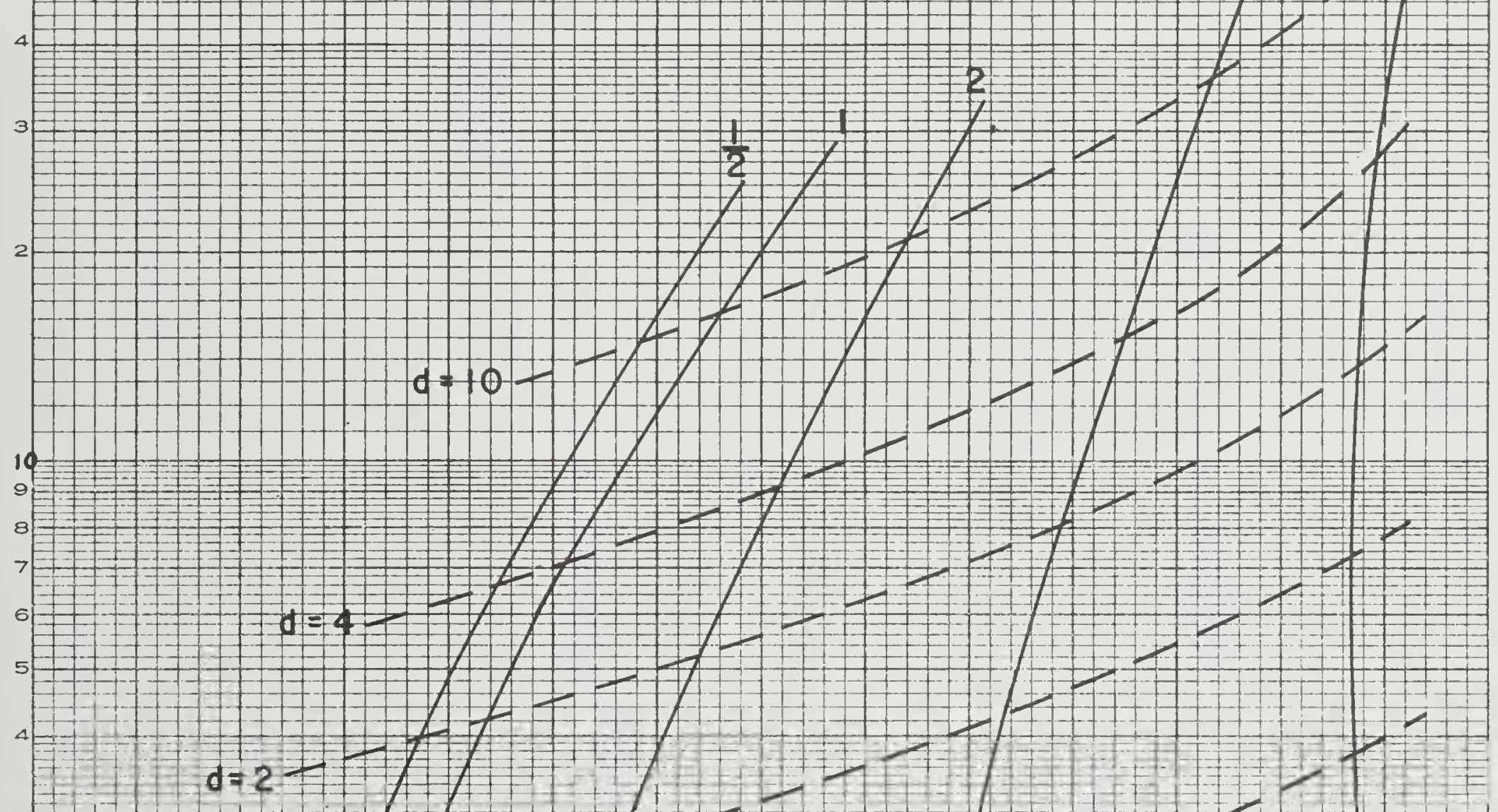


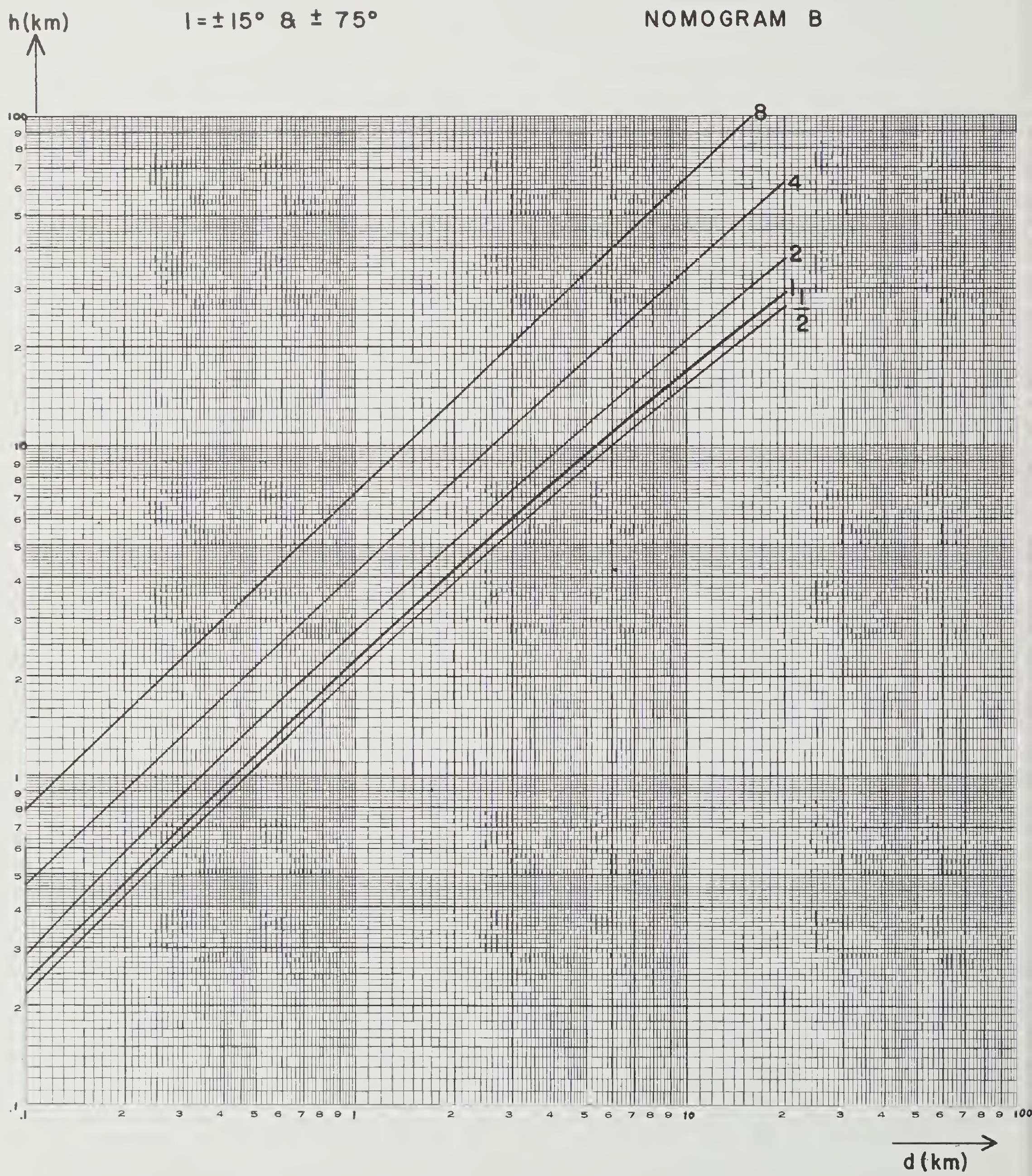




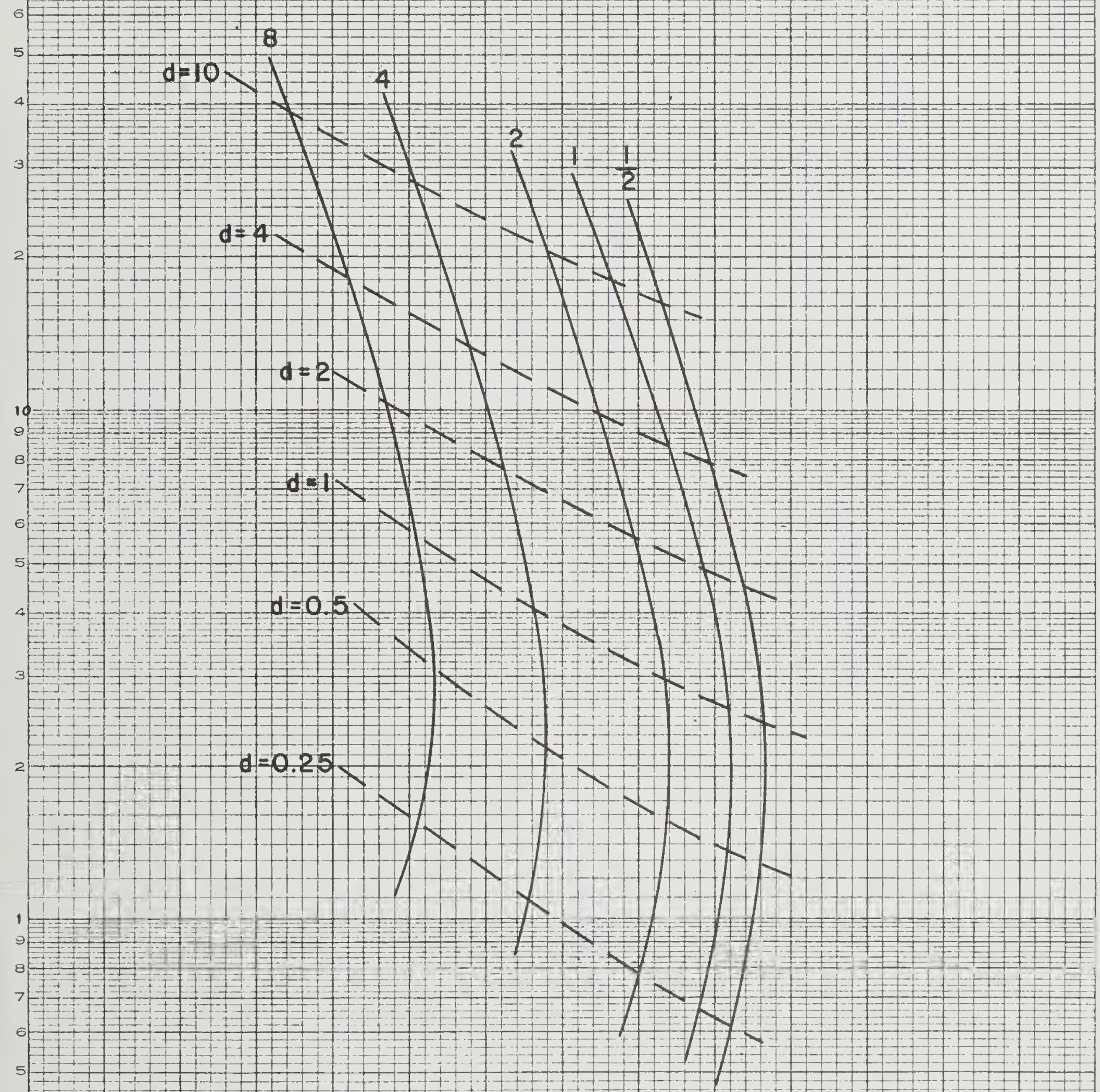

:

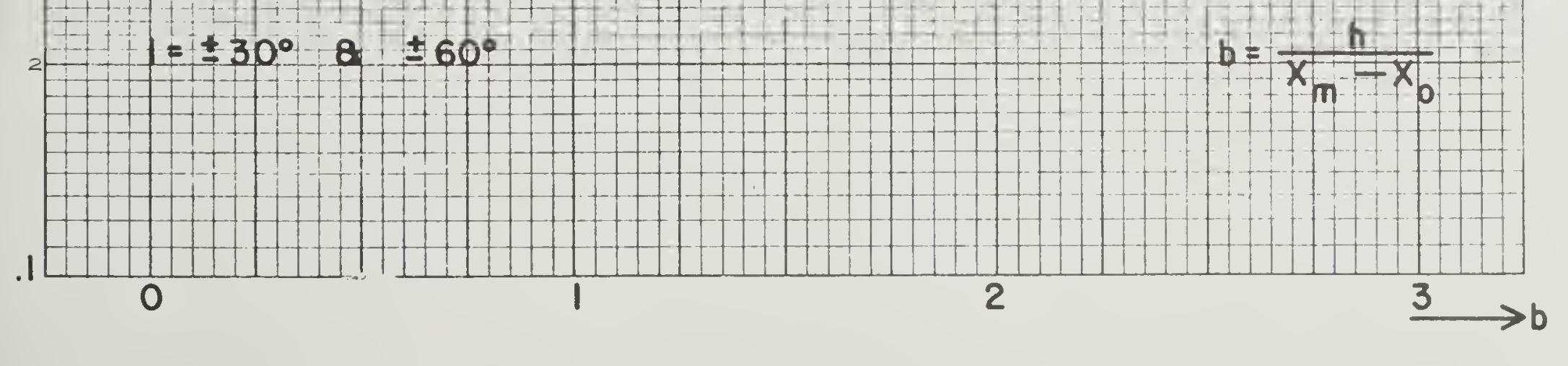




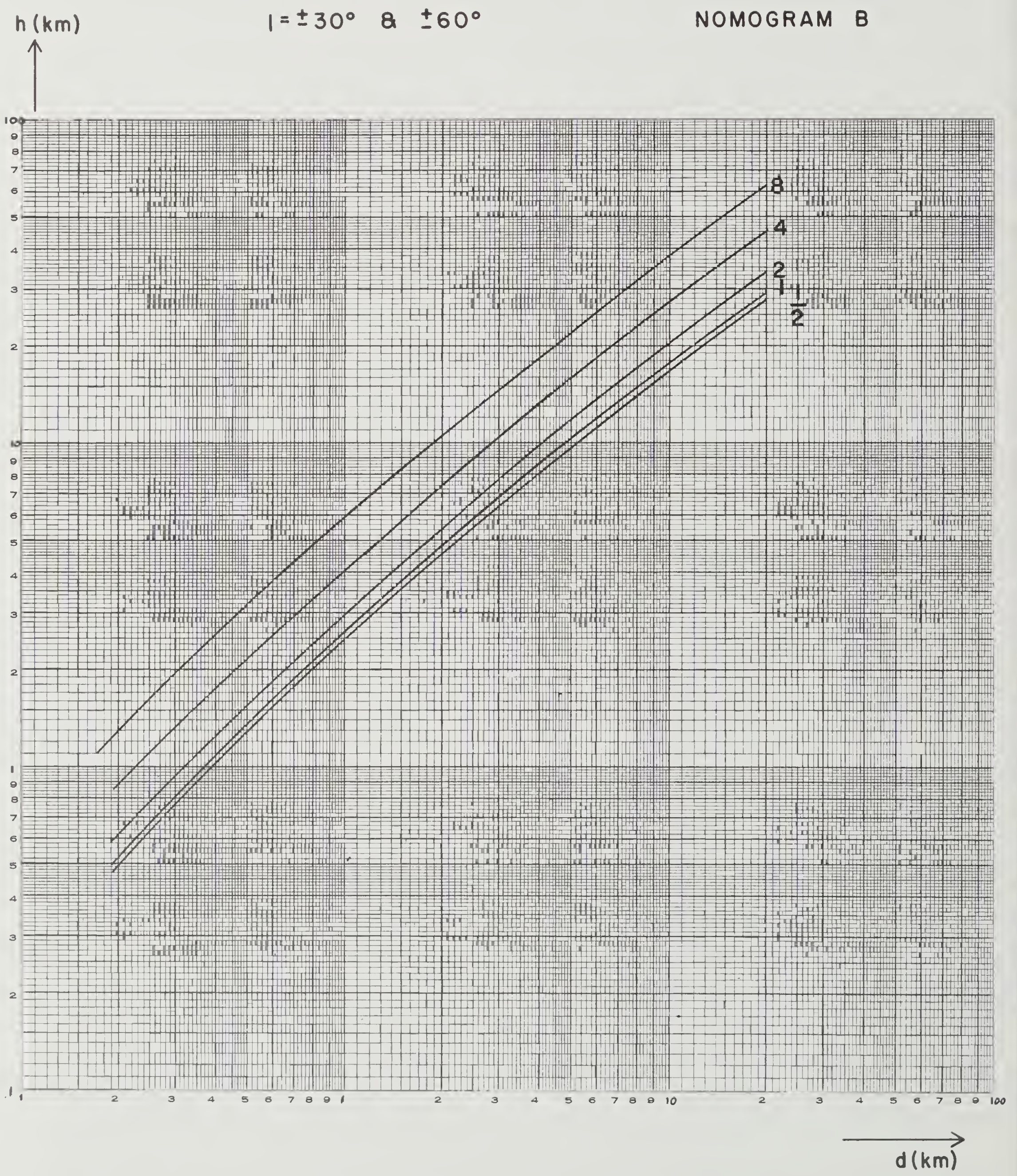

FIG. 4-35 


\section{$F 16: 4-36$}

NOMOGRAM A

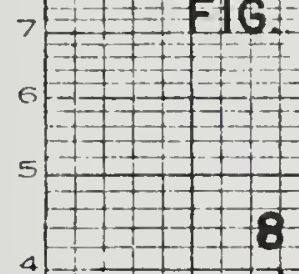

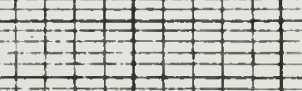

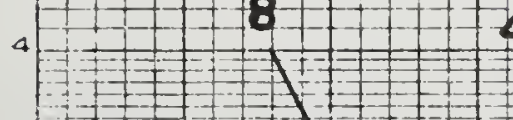

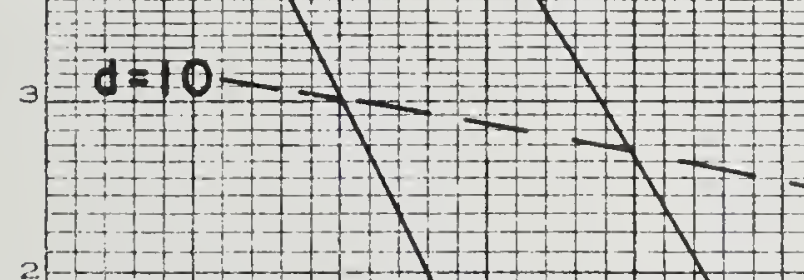
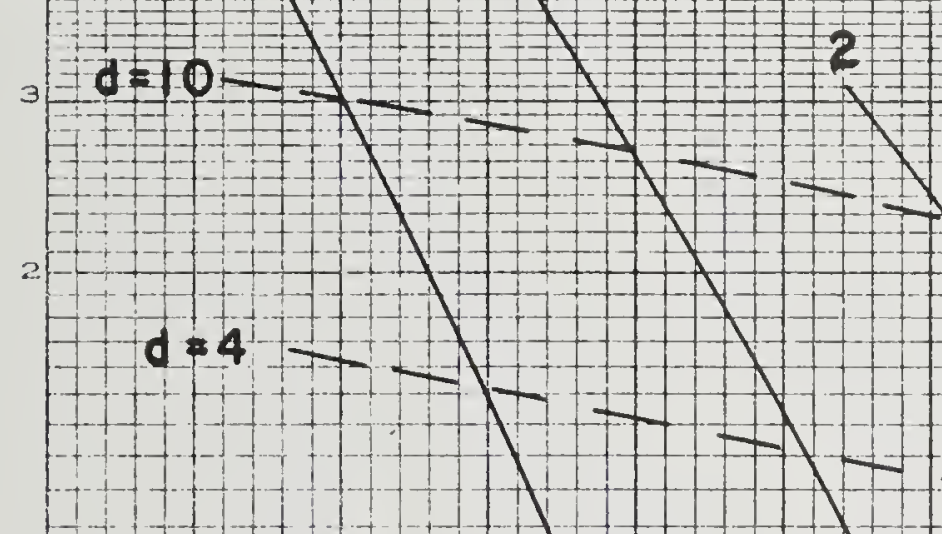

$d=2-+$

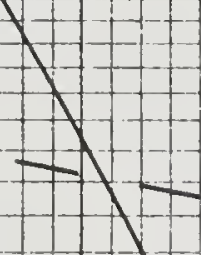

酯

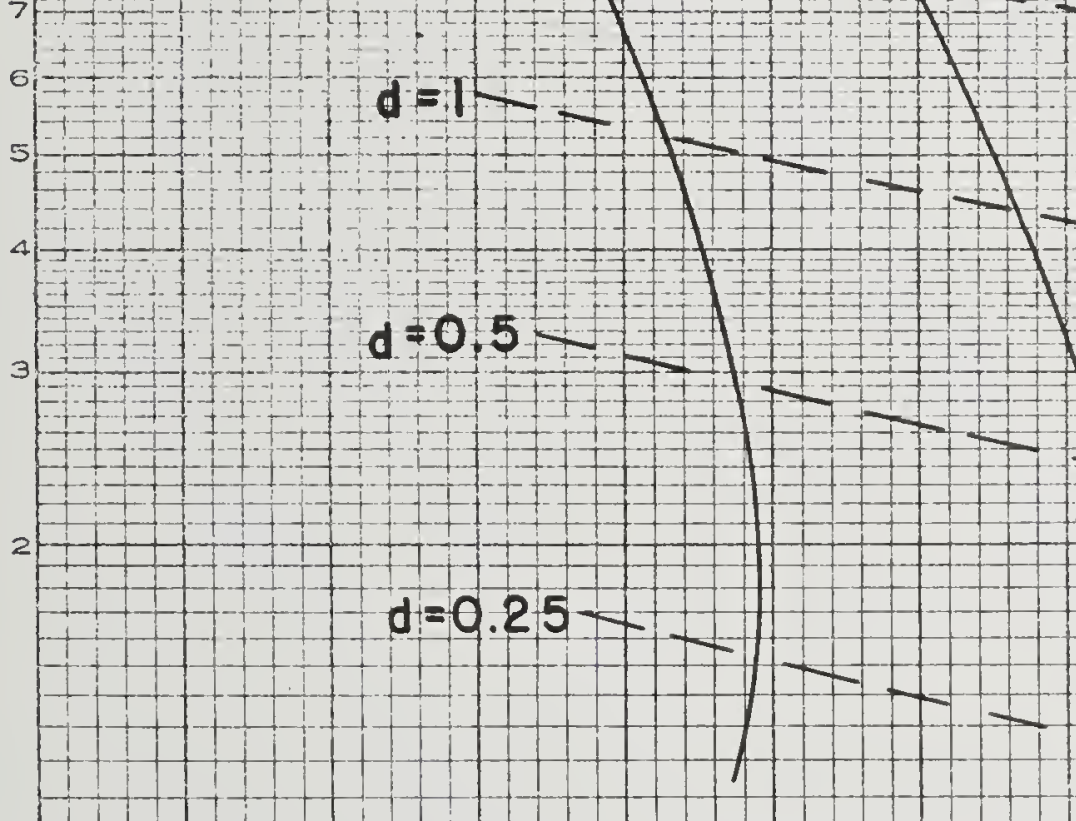

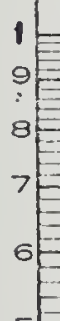

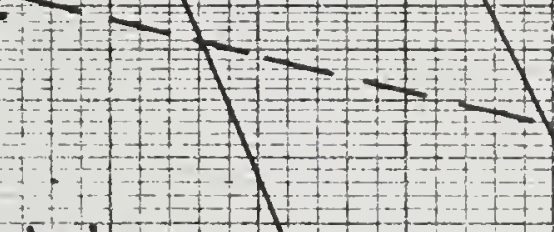

$1=\frac{1}{2} \frac{1}{4}$
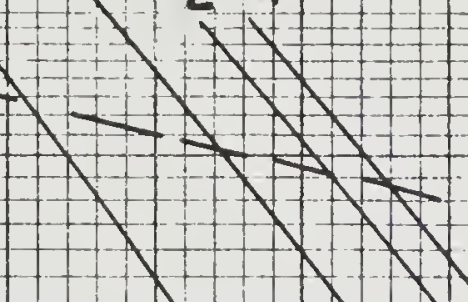

-

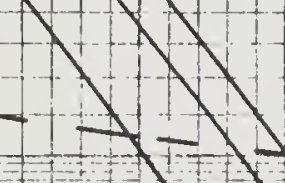

$-$
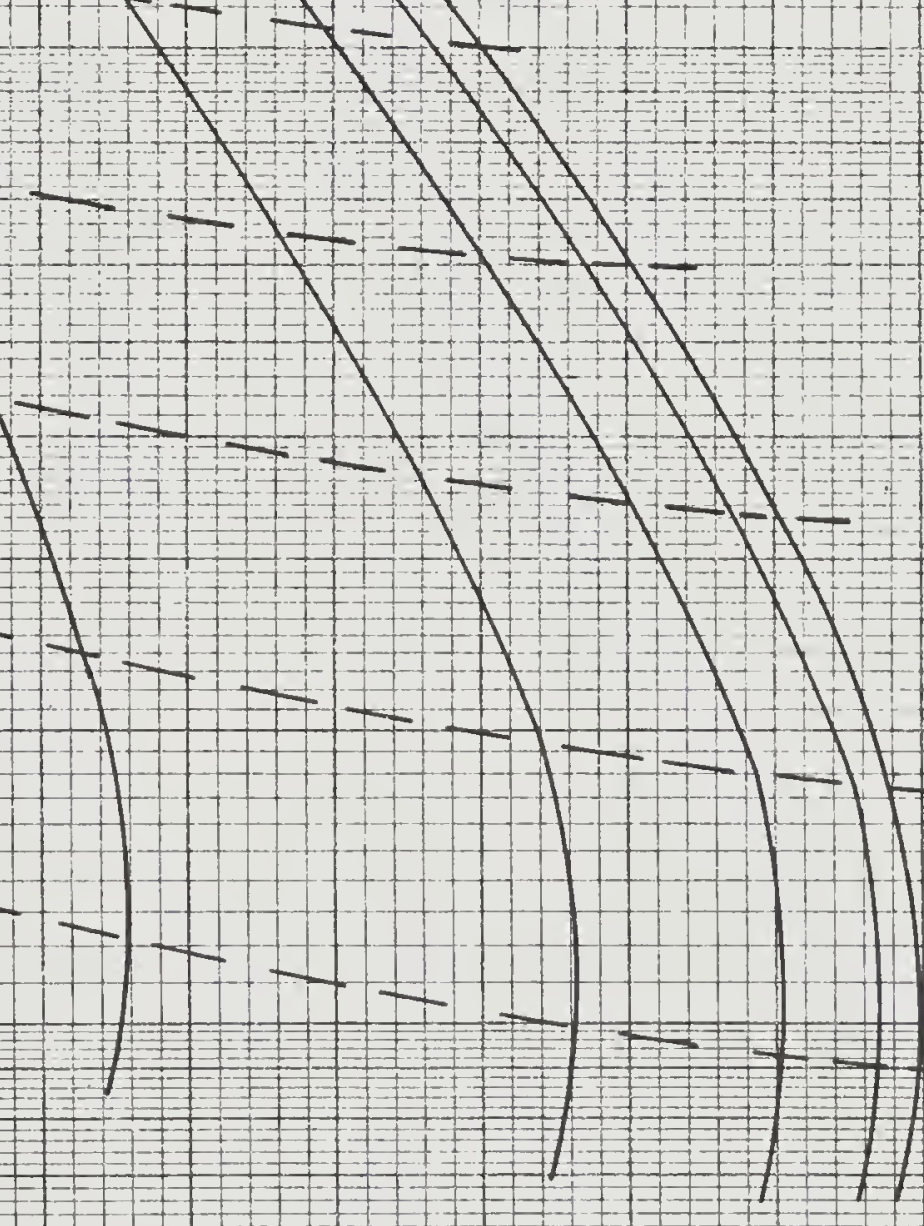

$5+1$

$4+1+-1-1-1$

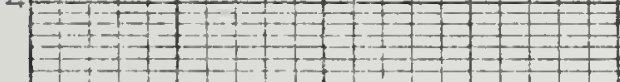

3

$2+1= \pm 45^{\circ}$

$$
b=\frac{h}{X_{m}-X_{0}}
$$




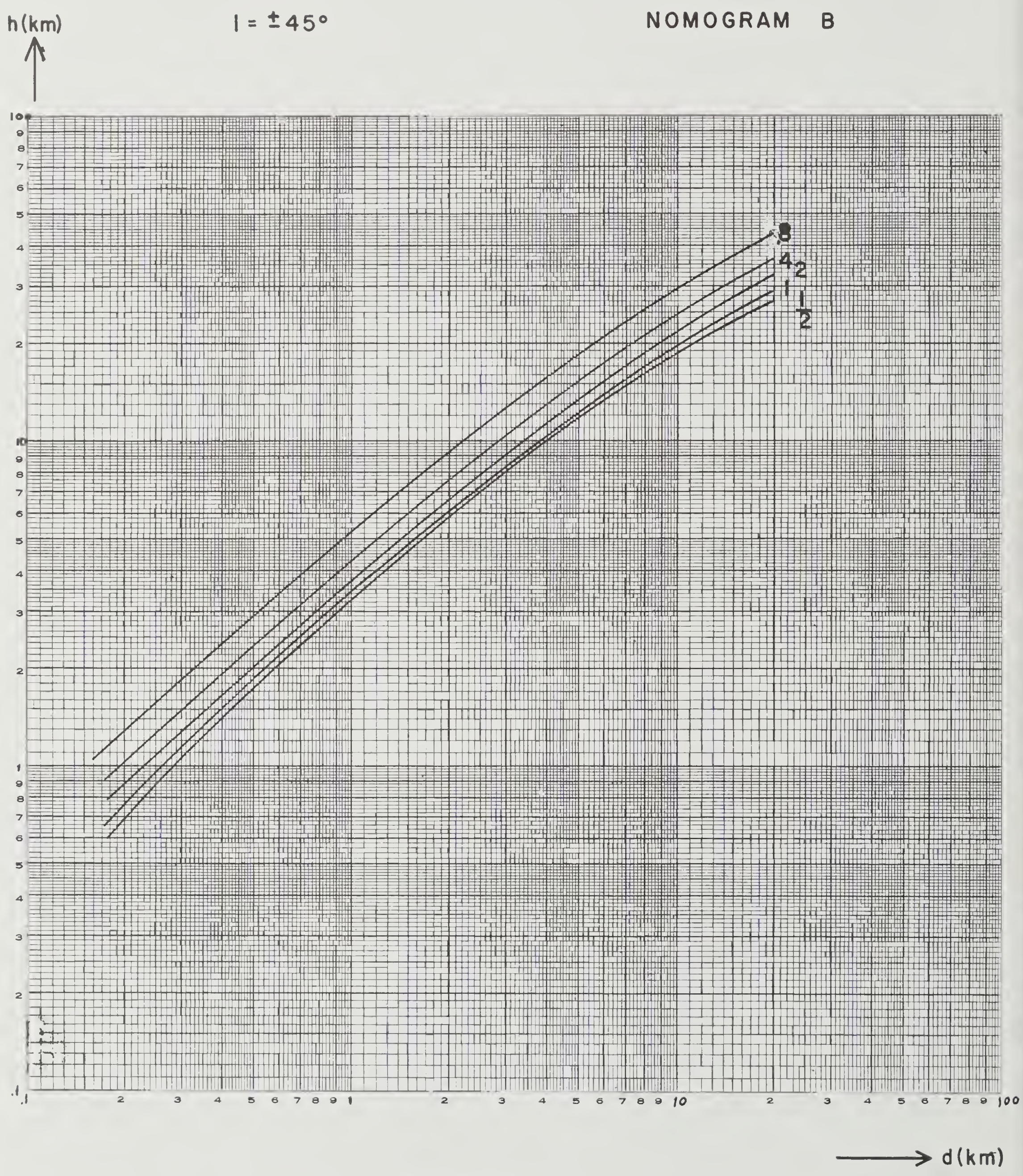

FIG. 4-37 


\section{Table 4-1}

Correction for the Strike: $w / d=5, d=2, D=15$

$\begin{array}{cccccc} & s & \Delta h(\%) & \Delta \mathrm{b}(\%) & \Delta \bar{h}(\%) & \Delta \bar{b}(\%) \\ 60^{\circ} & +1.8 & +2.8 & & \\ 45^{\circ} & +4.1 & +12.4 & & \\ 30^{\circ} & +10.0 & +26.9 & & \\ I 5^{\circ} & +14.4 & +23.4 & +11.4 & +14.4 \\ & 0^{\circ} & & & -4.2 & +15.9 \\ & & & & & \\ & 60^{\circ} & +3.1 & -3.3 & & -1.1 \\ 45^{\circ} & +4.4 & -10.9 & -1.1 \\ & 30^{\circ} & -1.3 & -33.4 & +4.4 & -11.7 \\ 15^{\circ} & & & -3.4 & +3.5 \\ 0^{\circ} & & & -14.7 & -7.2\end{array}$

\begin{tabular}{cccc}
\hline$=45^{\circ}$ & $60^{\circ}$ & +3.3 & +13.4 \\
$45^{\circ}$ & 0.0 & +21.6 \\
$30^{\circ}$ & -4.0 & +35.7 \\
$15^{\circ}$ & -13.1 & +33.4 \\
& $0^{\circ}$ & -16.5 & +19.7 \\
$I=60^{\circ} \quad$ & $60^{\circ}$ & -2.7 & +2.2 \\
& $45^{\circ}$ & -5.8 & +2.1 \\
& $30^{\circ}$ & -8.5 & +0.9 \\
& $15^{\circ}$ & -14.1 & -5.7 \\
& $0^{\circ}$ & -14.8 & -7.9 \\
& & & -10.4 \\
& $45^{\circ}$ & -1.5 & +2.9 \\
& $15^{\circ}$ & -5.1 & +19.7
\end{tabular}




$$
4-56
$$

Table 4-2

Correction for the Strike: $w / d=5, d=0.5, D=15$

\begin{tabular}{|c|c|c|c|c|c|}
\hline & s & $\Delta h(\%)$ & $\Delta b(\%)$ & $\Delta \bar{h}(\not)$ & $\Delta \bar{b}(\%)$ \\
\hline \multirow[t]{3}{*}{$I=15^{\circ}$} & $45^{\circ}$ & +2.0 & -1.5 & & \\
\hline & $15^{\circ}$ & +3.2 & -12.7 & $+4 \cdot 9$ & +2.8 \\
\hline & $0^{\circ}$ & & & -1.2 & +330 \\
\hline \multirow[t]{3}{*}{$I=30^{\circ}$} & $45^{\circ}$ & $+4 \cdot 2$ & $-4 \cdot 5$ & -6.5 & $-44 \cdot 0$ \\
\hline & $15^{\circ}$ & & & -0.8 & +10.7 \\
\hline & $0^{\circ}$ & & & +5.4 & +58.0 \\
\hline \multirow[t]{3}{*}{$I=45^{\circ}$} & $45^{\circ}$ & +6.4 & +34.0 & & \\
\hline & $15^{\circ}$ & -1.6 & +57.0 & & \\
\hline & $0^{0}$ & -3.1 & +123 & & \\
\hline \multirow[t]{3}{*}{$I=60^{\circ}$} & $45^{\circ}$ & +1.7 & +12.6 & & \\
\hline & $15^{\circ}$ & -3.2 & +18.6 & & \\
\hline & $0^{\circ}$ & $-4 \cdot 3$ & +60.5 & & \\
\hline \multirow[t]{3}{*}{$I=75^{\circ}$} & $45^{\circ}$ & -1.5 & +2.0 & & \\
\hline & $15^{\circ}$ & -1.9 & +72.4 & & \\
\hline & $0^{\circ}$ & -2.1 & +172 & & \\
\hline
\end{tabular}




\section{Table 4-3}

Correction for the Strike: w/d=1, $d=2, D=15$

\begin{tabular}{|c|c|c|c|c|c|}
\hline & $\mathrm{s}$ & $\Delta h(\%)$ & $\Delta b(\%)$ & $\Delta \bar{h}(\%)$ & $\Delta \bar{b}(\%)$ \\
\hline \multirow[t]{5}{*}{$I=15^{\circ}$} & $60^{\circ}$ & -2.6 & $-1 / 4.8$ & & \\
\hline & $45^{\circ}$ & -4.8 & -31.0 & & \\
\hline & $30^{\circ}$ & -12.6 & -42.1 & & \\
\hline & $15^{\circ}$ & $-31 \cdot 3$ & -72.8 & -30.0 & -71.0 \\
\hline & $0^{\circ}$ & & & +7.2 & +185 \\
\hline \multirow[t]{5}{*}{$I=30^{\circ}$} & $60^{\circ}$ & $-4 \cdot 3$ & -5.8 & & \\
\hline & $45^{\circ}$ & -12.8 & -19.3 & -30.8 & -37.9 \\
\hline & $30^{\circ}$ & -28.5 & $-37 \cdot 4$ & $-14 \cdot 3$ & -26.0 \\
\hline & $15^{\circ}$ & & & +8.8 & +21.6 \\
\hline & $0^{\circ}$ & & & +20.1 & +190 \\
\hline \multirow[t]{5}{*}{$I=15^{\circ}$} & $60^{\circ}$ & +7.4 & +15.0 & & \\
\hline & $45^{\circ}$ & +16.2 & $+24 \cdot 4$ & & \\
\hline & $30^{\circ}$ & +30.2 & +55.0 & & \\
\hline & $15^{\circ}$ & $+44 \cdot 0$ & +114 & & \\
\hline & $0^{\circ}$ & +52.0 & +306 & & \\
\hline \multirow[t]{3}{*}{$I=60^{\circ}$} & $45^{\circ}$ & +11.5 & +32.2 & & \\
\hline & $15^{\circ}$ & +20.0 & +99.4 & & \\
\hline & $0^{\circ}$ & +21.0 & +184 & & \\
\hline \multirow[t]{3}{*}{$I=75^{\circ}$} & $45^{\circ}$ & +1.9 & $+24 \cdot 5$ & & \\
\hline & $15^{\circ}$ & +3.0 & +91.7 & & \\
\hline & $0^{\circ}$ & +5.0 & +154 & & \\
\hline
\end{tabular}


Table $4-4$

Correction for the Strike: w/d=1, d=0.5, $D=15$

\begin{tabular}{|c|c|c|c|c|c|}
\hline & s & $\Delta h(\%)$ & $\Delta b(\%)$ & $\Delta \bar{h}(\%)$ & $\Delta \bar{b}(\not{p})$ \\
\hline \multirow[t]{3}{*}{$I=15^{\circ}$} & $45^{\circ}$ & -5.0 & -37.0 & & \\
\hline & $15^{\circ}$ & -35.6 & -81.7 & -29.2 & -75.8 \\
\hline & $0^{\circ}$ & & & +4.6 & +400 \\
\hline \multirow[t]{3}{*}{$I=30^{\circ}$} & $45^{\circ}$ & -14.0 & -23.6 & $-34 \cdot 2$ & -41.7 \\
\hline & $15^{\circ}$ & & & +10.6 & +19.6 \\
\hline & $0^{\circ}$ & & & +25.0 & +440 \\
\hline \multirow[t]{3}{*}{$I=45^{\circ}$} & $45^{\circ}$ & +19.2 & +23.8 & & \\
\hline & $15^{\circ}$ & +58.0 & +188 & & \\
\hline & $0^{\circ}$ & +60.7 & +730 & & \\
\hline \multirow[t]{3}{*}{$I=60^{\circ}$} & $45^{\circ}$ & +12.5 & +23.6 & & \\
\hline & $15^{\circ}$ & +22.7 & +160 & & \\
\hline & $0^{\circ}$ & +23.8 & +396 & & \\
\hline \multirow[t]{3}{*}{$I=75^{\circ}$} & $45^{\circ}$ & +1.8 & +39.4 & & \\
\hline & $15^{\circ}$ & +5.6 & +190 & & \\
\hline & $0^{\circ}$ & +6.5 & +360 & & \\
\hline
\end{tabular}




\section{Appendix}

\section{A Digital Computer Program \\ for the Computation of Magnetic Anomalies over Two-Dimensional Polygonal Bodies \\ M. Talwani and J.R. Heirtzler}

\section{Framing the Problem}

Rewrite eqs (2-7) and (2-8) for a prism of Fig. (2-4) expressing $\phi$ in terms of distance:

$$
\begin{aligned}
& x_{1}-x_{2}=x_{12}=-x_{21}, z_{1}-z_{2}=z_{12}=-z_{21}, \\
& \sin \phi=\frac{z_{21}}{\left(z_{21}^{2}+x_{12}^{2}\right)^{1 / 2}, \cos \phi=\frac{x_{12}}{\left(z_{21}^{2}+x_{12}^{2}\right)^{1 / 2}},} \\
& \sin \phi \cos \phi=\frac{z_{21} x_{12}}{z_{21}^{2}+x_{12}^{2}}=\frac{z_{12}^{2} x_{21}}{z_{12}^{2}+x_{21}^{2}}, \\
& \sin ^{2} \phi=\frac{z_{21}^{2}}{z_{21}^{2}+x_{12}^{2}}=\frac{z_{12}^{2}}{z_{12}^{2}+x_{21}^{2}} \cdot
\end{aligned}
$$


This jields

$$
\begin{aligned}
& V=2\left(M_{x} Q^{\prime}-M_{z} P^{\prime}\right) \\
& H=2\left(M_{x} P^{\prime}+M_{z} Q^{\prime}\right)
\end{aligned}
$$

with

$$
\begin{aligned}
& P^{\prime}=\frac{z_{31}^{2}}{z_{21}^{2}+x_{12}^{2}}\left(\theta_{2}-\theta_{1}\right)-\frac{z_{21} x_{12}}{z_{21}^{2}+x_{12}^{2}} \log \left(r_{2}, r_{1}\right) \\
& Q^{\prime}=\frac{z_{11} x_{12}}{z_{21}^{2}+x_{12}^{2}}\left(\theta_{2}-\theta_{0}\right)+\frac{z_{21}^{2}}{z_{21}^{2}+x_{12}^{2}} \log \left(r_{2} / r_{1}\right)
\end{aligned}
$$

By refering back to page 2-7, it is seen that integrations leading to this result were carried out in a counter-clockwise direction, that is $\theta$ moving c.c.w. during the integration. For a computer program there is an advantage in using a different labeling of body points (see Fig. A-I):

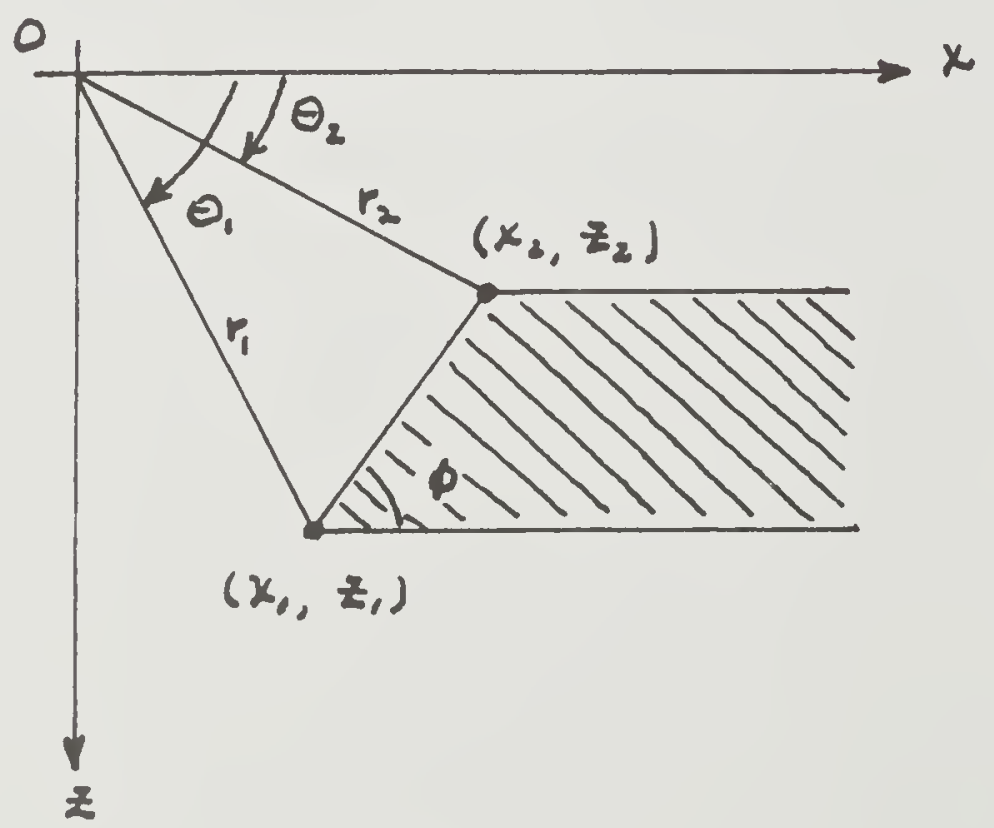

Fig. A-I 
The previous calculations can be utilized provided we replace $r_{1}$ by $r_{2}, r_{2}$ by $r_{1}, \theta_{1}$ by $\theta_{2}$, and $\theta_{2}$ by $\theta_{1}$. These changes give the following equations:

$$
\begin{aligned}
& V=z\left(M_{x} Q-M_{z} P\right) \\
& H=2\left(M_{x} P+M_{z} Q\right)
\end{aligned}
$$

$$
\begin{aligned}
& P=\frac{z_{21}^{2}}{z_{21}^{2}+x_{12}^{2}}\left(\theta_{1}-\theta_{2}\right)+\frac{z_{21} x_{12}}{z_{21}^{2}+x_{12}^{2}} \log \left(r_{2} / r_{1}\right) \\
& Q=\frac{z_{21} x_{12}}{z_{21}^{2}+x_{12}^{2}}\left(\theta_{1}-\theta_{2}\right)-\frac{z_{21}^{2}}{z_{21}^{2}+x_{12}^{2}} \log \left(r_{2} / r_{1}\right)
\end{aligned}
$$

For a given field point (point of observation or origin location) the computer computes eqs $(A-5),(A-6)$, and the total intensity anomaly (oq 2-9) for each face of the body using eqs (A-7) and (A-8) as internal subroutines. It sums the anomelies for each consecutive face and then proceeds to do the same for other bodies present. After the various sums are printed for a given field point it goes to the next field point, etc.

The program has been written specifically for bodies with induced magnetization only, but can also.be used without modification for bodies having remanent magnetization or bodies with both remanent and induced magnetization. 
For computing convenience we can write $(A-5$ and $(A-6)$ above as:

$$
\begin{aligned}
& V=2 k F\left[\left(\cos I^{\prime} X \sin s^{\prime}\right) Q-\left(\sin I^{\prime}\right) P\right] \\
& H=2 k F\left[\left(\cos I^{\prime}\right)\left(\sin s^{\prime}\right) P+\left(\sin I^{\prime}\right) Q\right]
\end{aligned}
$$

For bodies with induced magnetization only, $k$ represents the susceptibility and $F$ the magnitude of the total undisturbed field. $I^{\prime}=I, s^{\prime}=s$ as in Fig. 2-7. For bodies with remanent or mixed magnetization $\mathrm{kF}$ has, of course, no significance; it is merely retained for convenience so that one can use the same program as for bodies with induced magnetization. In practic $\mathrm{kF}$ is made to represent the magnitude of $M_{\text {rem }}$ for bodies with remanent magnetization by giving $k$ the dummy value of $I$ and $F$ the value for $M_{\text {rem }}$. Also I' $=a, s=b$ as mentioned on $p \cdot 2-11$. Similarly for a body with mixed magnetization $k$ is again given the dummy value of $I$ and $F$ the value of $M_{\text {tot }}$. It $=\alpha, s^{\prime}=\beta$ as mentioned on p. $2-12$.

We note that the expression (2-9) for the evaluation of T, the total anomaly, remains unchanged whether the magnetization is induced, remanent, or mixed.

2. Flow Diagram

To assist in the understanding of the flow diagram and the subsequent program the following list of symbols is defined: 


\section{Table A-I}

\section{Program Symbols}

Symbol

DIP\%

DIPD:

D*

DD*

F*

C*

EXX, ZEE*

JTOT\%

CONS*

F0\%

DELX*

THETA, THETB

OMEGA

K

KTOT*

INOE
Moaning

Inclination or Dip, $I$, in degrees

Modifled Inclination or Dip, Is in degrees

Strike, $s$, in degrees

Modified Strike, s', in degrees

Total Magnetic Field intensity, In gammas

Magnetic susceptibility, $k$, in $\theta_{.} \cdot u_{\text {. }}$ Coordinates of body corners, entered in program in clockwise sequence including first twice

Total number of body points including first twice

Constant value of $z$ of field points, zero for shipboard surveys, negative for aeromagnetic surveys taking sea level as $z=0$

$x$ value of first field point Increment in $x$ value of field points Angle to corner measured from $x$-axis, Angular span of face from field point, $\theta_{1}-\theta_{2}$

Subscript for consecutive field points

Total number of field points

Subscript for body number 
LNOT*

$\mathrm{H}$

V

PSUM

QS UM

HSUM, VSUM

HASUM, VASUM

TSOM

TASUM
Total number of bodies

Horizontal anomaly for single face of body at fleld point, in gammas

Vertical anomaly for single face of body at field point, in gammas

The sum of $\mathrm{P}^{\mathrm{S}} \mathrm{s}$ for the varlous faces of the body

The sum of $Q^{\prime} s$ for the various faces of the body

Horlzontal and Vertical anomalies, in gammas, at field point for given body

Horizontal and vertical anomalies, in gammas, at field point for all bodies

Anomaly in total intensity, in gammas, at fleld point for given body Anomaly in total intensity, in gammas, at fleld point for all bodies

*Data which must be entered into computer from typewriter.

$D$ and DIP are used in the calculation of $V$ and $H$.

DD and DIPD are used to calculate TSUM and TASUM. 

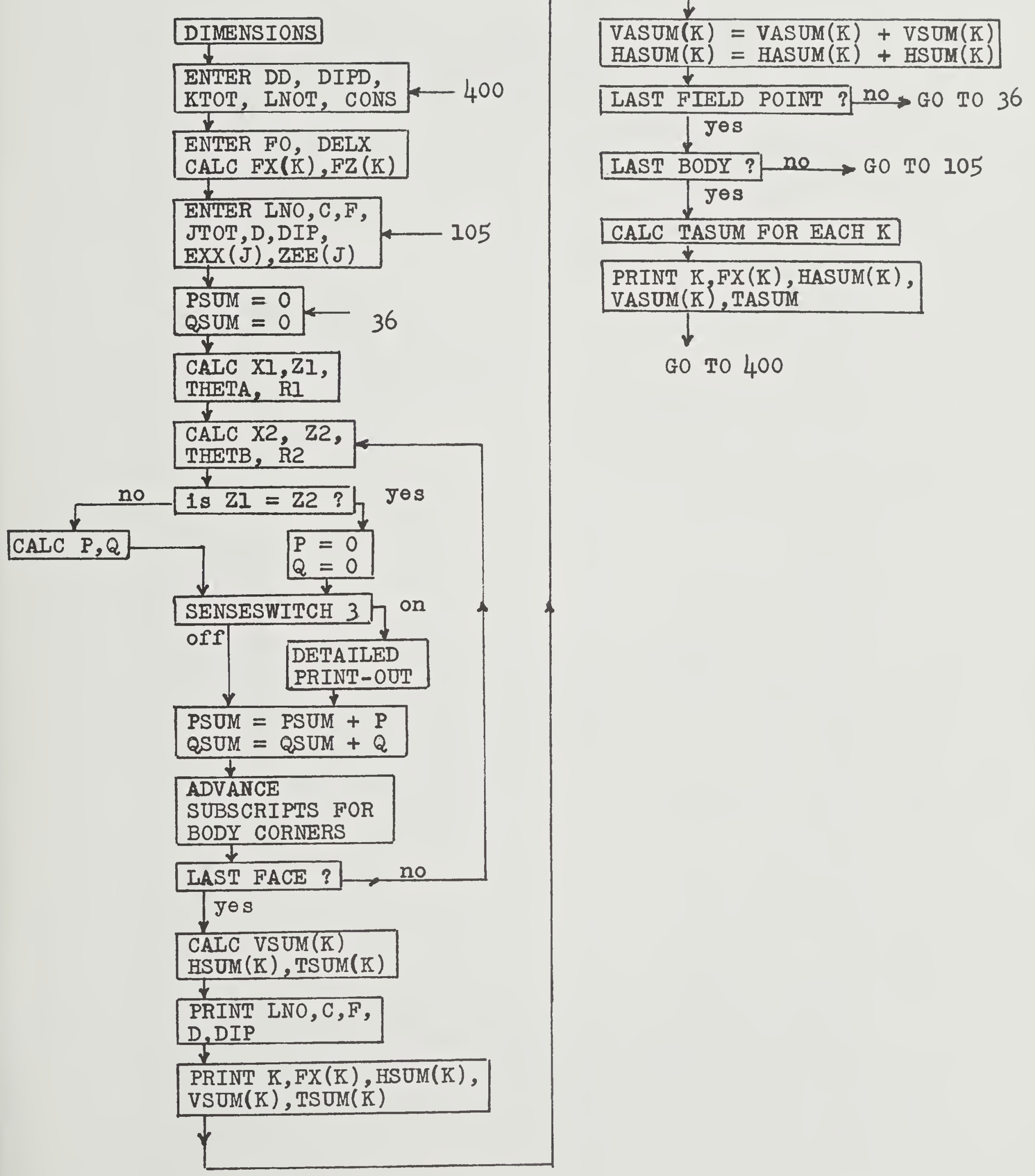
C FORTRAN PROGRAM FOR IBM 1620

DIMENSION FX (47), FZ (47), VASUM (47), HASUM (47)

DIMENSION VSUM (47), HSUM $(47), \operatorname{EXX}(30), \mathrm{ZEE}(30)$

400 ACCEPT, DD, DIPD, KTOT, LNOT, CONS

$402 \operatorname{SDD}=\operatorname{SIN}(.0174533 \% \mathrm{DD})$

$\operatorname{CDIPD}=\operatorname{COS}(.0174533 * D I P D)$

$\operatorname{SDIPD}=\operatorname{SIN}(.0174533 * D I P D)$

601 ACCEPT, FO, DELX

603 DO $604 \mathrm{~K}=1$, KTOT

$\mathrm{RK}=\mathrm{K}$

$F X(K)=(F O-D E L X)+D E L X * R K$

$\mathrm{FZ}(\mathrm{K})=\mathrm{CONS}$

$\operatorname{VASUM}(K)=0$.

$604 \operatorname{HASUM}(K)=0$.

105 ACCEPT, LNO, C, F, JTOT

ACCEPT, D,DIP

$410 S D=\operatorname{SIN}(.0174533 * D)$

$\operatorname{CDIP}=\operatorname{COS}(.0174533 \% D I P)$

$\operatorname{SDIP}=\operatorname{SIN}(.0174533 \% D I P)$

DO $11 \mathrm{~J}=1$, JTOT

411 ACCEPT, EXX (J), ZEE (J)

11 CONT INUE

DO $36 \mathrm{~K}=1$, KTOT

PSUTM $=0$.

QSUM $=0$.

$X I=E X X(I)-F X(K)$

$\mathrm{ZI}=\mathrm{ZEE}(\mathrm{I})-\mathrm{FZ}(\mathrm{K})$

$\mathrm{RSQI}=\mathrm{XI} * * 2+21 * * 2$

IF (XI) $110,140,180$

110 IF (ZI) $120,130,130$

120 THETA $=A T N(Z 1 / X I)-3.1415927$

GO TO 200

130 THETA=ATN $(\mathrm{ZI} / \mathrm{XI})+3.1415927$

GO TO 200

$140 \operatorname{IF}(\mathrm{ZI}) 150,160,170$

150 THETA $=-1.5707963$

GO TO 200

160 THETA $=0.0$

GO TO 200

170 THETA=1.5707963

GO TO 200

180 THETA $=A T N(\mathrm{ZI} / \mathrm{XI})$

$200 \mathrm{~J}=2$

$201 \times 2=\operatorname{FXX}(\mathrm{J})-F X(\mathrm{~K})$

$\mathrm{Z} 2=\mathrm{ZEME}(\mathrm{J})-\mathrm{FZ}(\mathrm{K})$

$\mathrm{RSQ} 2=\mathrm{X} 2 * \div 2+\mathrm{Z} 2 * 2$

IF(X2) $210,240,280$

$210 \operatorname{IF}(\mathrm{Z} 2) 220,230,230$

220 THETB=ATN $(\mathrm{Z} 2 / \times 2)-3.1415927$

GO TO 300

230 THETB $=A T N(Z 2 / X 2)+3.1415927$

GO TO 300 


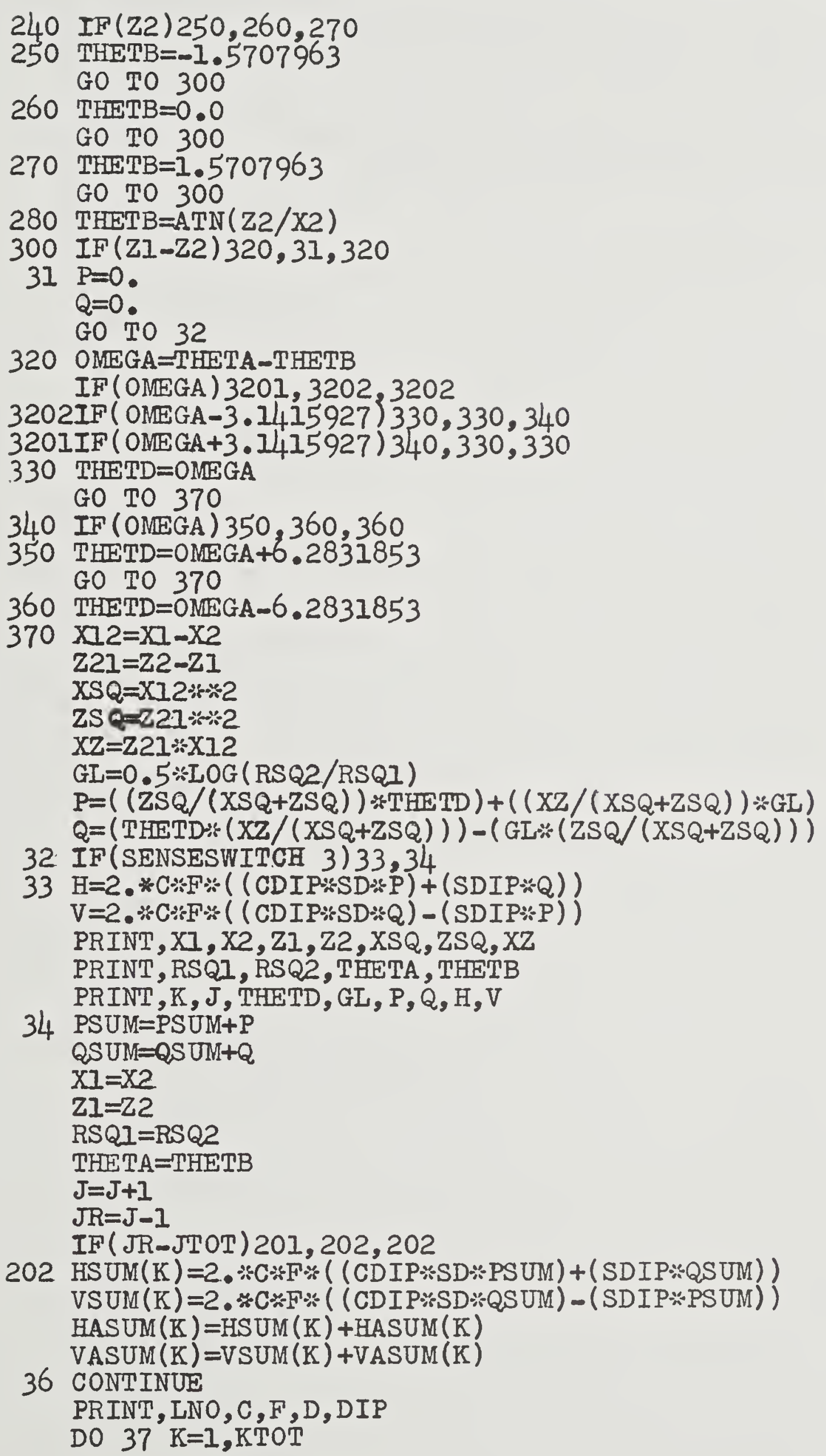


TSUM $=(\operatorname{HSUM}(K) * C D I P D * S D D)+(\operatorname{VSUM}(K) * S D I P D)$

37 PRINT, K, FX $(K)$, HSUM $(K)$, VSUM $(K)$, TSUM

IF (LNO-LNOT) $105,38,105$

$38 \mathrm{~K}=1$

381 TASUM $=(\operatorname{HASUM}(K) * C D I P D * S D D)+($ VAS UM $(K) * S D I P D)$

39 PRINT, $K, F X(K)$, HASUM $(K)$, VASUM $(K)$, TASUM

$\mathrm{K}=\mathrm{K}+\mathrm{I}$

$\mathrm{KR}=\mathrm{K}-1$

IF (KR-KTOT) $381,391,391$

391 GO TO 400

END 
3. A Simple Example

Suppose we wish to determine the induced anomalies of three field points for an assumed body shape as given in Fig. A-2.

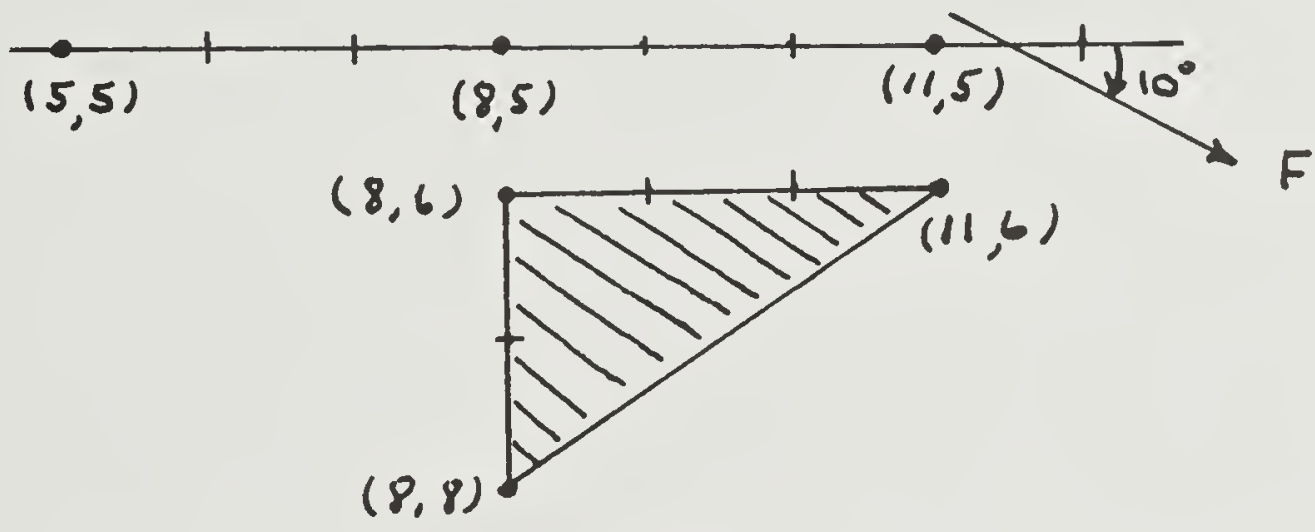

Fig. A-2

Suppose the axis of the body makes an angle of $60^{\circ}$ with magnetic north, the inclination is $10^{\circ}$, the ambient magnetic field strength is 50,000 gammas, the susceptibility is 0.001 emu.

We have JTOT $=4$, LNO $=1$, LNOT $=1$, CONS $=5 .$, KTOT $=3$, FO $=5 .$, DELX $=3$.

Information in the following form must be supplied to the machine by typewriter

60. 10.315 .

5. 3 .

1.00150000 .4

60. 10 .

8.8 .

8. 6 .

11. 6 .

8.8. 


\section{$A-12$}

With senseswitch 3 off numerical results will be printed as follows:

(LNO)

(F)

(D)

(DIP)

$1 \quad 1.0000000 \mathrm{E}-03$

50000.000

60.000000

10.000000

(K) $\quad F X(K)$

$\operatorname{HSUM}(\mathrm{K})$

$\operatorname{VSUM}(\mathrm{K})$

$\operatorname{TSUM}(K)$

15.0000000

11.142248

8.2548530

10.936315

28.0000000

$-25.503102$

53.009137

$-12.545855$

311.000000

$\begin{array}{lll}-12.179321 & -43.110189 & -17.873370\end{array}$ 


\section{$A-13$}

4. Console Operation for Lamont IBM 1620

Switches:

Senswitch 3 on for detailed print out (used

for trouble shooting only)

Other senseswitches OFF

Program switches OFF

Other switches ON

Clear Memory: Reset, Insert, 160001000000, Release, Start, (after a few seconds) Instant Stop.

Load Tape: Mount object tape, set tape console switch to Reel. Reset, Insert, 360000000300 , Release, Start. If machine fails to read, repeat previous instructions. If functioning properly machine will pause $2 / 3$ way through reading to think but will proceed to end of tape when it will type "Load Data".

Load Data: Set typewriter tabs at approximately

$\begin{array}{lllll}16 & 28 & 45 & 58 & 70\end{array}$

Reset, Insert, 4907500, Release, Start

Numbers must be typed in upper case.

Minus signs and decimal points in lower case. Zero in either case.

A space must be typed between successive data within a line of data.

A record mark after the last datum of a line is optional. 
A decimal point is required after floatingpoint data.

A decimal point is optional after fixedpoint data.

Typing Error: If incorrect data has been entered in machine Reset, Insert, 4907500, Release, Start, reenter all data.

If incorrect data has been typed but not entered into machine Senswitch 4 ON, Release, Start, Senswitch 4 OFF, repeat line of data.

Stop Output: Deprese Stop. Neatness can be achieved by depressing Stop while carriage of typewriter is in return motion following typing of last line of desired data. If machine is not stopped it will automatically return to the stage where it is again ready to accept all data.

Check Stops: If a check stop occurs during data entry it may be due to a parity error in that part of the memory related to data storage: Reset, Insert, 4907500, Release, Start, re-enter all data.

If a check stop occurs at any other time or if the above procedure fails clear memory, re-load tape, re-enter all data. 


\section{ACKNOWLEDGEMENTS}

The figures of Chapters III and IV of this report were drawn by Jay Maxon and David Wolfe.

The aeromagnetic maps of Chapter IV-D were reproduced with the permission of the Geological Survey of Canada.

Financial support for this work was provided by the Office of Naval Research, Contract Nonr $266(48)$ and the National Science Foundation, Grant 4151.

Reproduction of this document in whole, or in part, Is permitted for any purpose of the United States Government.

\section{REFERENCES}

Baranov, V. (1953): Sur le calcul de l'influence gravimetrique des structures définies par les isobathes. Geoph. Prosp., v. 1, no. I, pp. 36-43.

Baranov, V. (1955): A new method of interpretation of aeromagnetic maps; pseudo-gravimetric anomalies. Geophysics v. 20, no. 2, pp. 379-380.

Egyed, L. (1948): The determination of an infinite inclined dike from the results of gravity and magnetic surveys. Geophysics v. 13, no. 3, pp. 437-442.

Gassmann, F. (195I): Graphical evaluation of the anomalies of gravity and of the magnetic field, caused by three- 
dimensional bodies. Proc. 3rd World Petrol, Congress, Sec. 1, pp. 613-621.

Girdler, R.W., and G. Peter (1960): An example of the importance of natural remanent magnetization in the interpretation of magnetic anomalies. Geophys. Prosp, v. 8, no. 3, pp. $474-483$.

Gulatee, B.L. (1938): Magnetic anomalies, Survey of India, Prof. paper no. 29, 12 p., 11 plates.

Haalck, H. (1927): Die magnetischen Verfahren der angewandten Geophysik. Borntraeger, Berlin.

Heiland, C.A. (1946): Geophysical exploration. PrenticeHall Inc., New York.

Heirtzler, J.R., C.L. Drake and J. Hirshman (1962): Magnetic anomalies off the east coast of North America. In preparation.

Henderson, R.G. and I. Zietz (1948): Analysis of total magnetic intensity anomalies produced by point and line sources. Geophysics, v. 13, no. 3, pp. 428-436. Henderson, R.G. and I. Zietz (1949a): The computation of second vertical derivatives of geomagnetic fields. Geophysics v. 14, no. 4, pp. 508-516.

Henderson, R.G. and I. Zietz (1949b): The upward continuation of anomalies in total magnetic intensity fields. Geophysics v. 14, no. 4, pp. 517-534.

Henderson, R.G. and I. Zietz (1957): Graphical calculation of total-intensity anomalies of three-dimensional bodies. Geophysics v. 22, no. 4, pp. 887-904. 
Henderson, R.G. and I. Zietz (1958): Magnetic-doublet theory in the analysis of total-intensity anomalies. Geol. Surv. Bull. 1052-D, Washington.

Henderson, R.G. (1960): A comprehensive system of automatic computation in magnetic and gravity interpretation. Geophysics $\nabla .25$, no. 3, pp. 569-585.

Hughes, D.S. and W.L. Pondrom (1947): Computation of vertical magnetic anomalies from total magnetic field measurements. Trans. Amer. Geophys. Union, v. 28, no. 2, pp. 193-197.

Köhler, K. (1958): Grundlagen für de Auswertung von magnetischen Anomalien. Freiberger Forschgshefte C4I, Akademie-Verl., Berlin, pp. 1-128.

Nettleton, L. (1942): Gravity and magnetic calculations. Geophysics v. 7, no. 3, pp. 293-310.

Nettleton, L. (1954): Regionals, residuals and structures. Geophysics v. 19, no. 1, pp. 1-22.

Peters, L.J. (1949): The direct approach to magnetic interpretation and its practical application. Geophysics v. 14, no. 3, pp. 290-320.

Skeels, D.C. (1947): Ambiguity in gravity interpretation. Geophysics v. 12, no. 1, pp. 43-56.

Skeels, D.C. and R.J. Watson (1949): Derivation of magnetic and gravitational quantities by surface integration. Geophysics v. 14, no. 2, pp. 133-150. 
Smellie, D.W. (1956): Elementary approximations in aeromagnetic interpretation. Geophysics v. 21, no. 4, pp. 1021-1040.

Smith, R.A. (1959): Some depth formulae for local magnetic and gravity anomalies. Geop. Prosp., v. 7, no. 1, pp. 55-63.

Smith, R.A. (1961): Some theorems concerning local magnetic anomalies. Geoph. Prosp., v. 9, no. 3, pp. 399-410.

Talwani, M., and M. Ewing (1960): Rapid computation of gravitational attraction of three-dimensional bodies of arbitrary shape. Geophysics v. 25, no. 1, pp. 203-225.

Vacquier, V., N.C. Steenland, R.G. Henderson and I. Zietz (1951): Interpretation of aeromagnetic maps. GSA Memoir 47 , $151 \mathrm{p}$

Vacquier, V., A.D. Raff and R.E. Warren (1961): Horizontal displacements in the floor of the northeastern Pacific Ocean. GSA Bulletin v. 72, no. 8, pp. 1251-1258. Vajk, R. (195I): Regional correction of gravity data. Geofisica Pura e Applicata, v. 19, fasc. 3-4, pp. 129-143.

Vestine, E.H. (1941): On the analysis of surface magnetic fields by integrals, part I. Terr. Mag. v. 46, no. I, pp. $27-41$

Vestine, E.H. and N. Davids (1945): Analysis and interpretation of geomagnetic anomalies. Terr. Mag. v. 50, no. 1, pp. $1-36$

Zietz, I. and R.G. Henderson (1954): Total-intensity magnetic anomalies of three-dimensional distribution by means 
of experimentally derived double layer model fields. Science, Washington, v. 119, no. 3088, pp. 329-330.

List of maps used

Geological Survey of Canada (1922): Portions of the districts of Algoma, Sudbury and Timiskaming, Ontario, map 155A.

Geological Survey of Canada (1945): Geological map of the Dominion of Canada, map 820A.

Geological Survey of Canada (1949): Geological map of the Maritime Provinces, map 910A.

Geological Survey of Canada (1955): Port Hawkesbury, N.S. Aeromagnetic series, sheet $11 \frac{\mathrm{F}}{\mathrm{II}}$, map $238 \mathrm{G}$.

Geological Survey of Canada (1956): Deskenatlata Lake South, NW Territories. Aeromagnetic series, sheet $85 \frac{\mathrm{A}}{16}$, map 107G.

Province of Ontario, Department of Mines (1955): Aeromagnetic map, Bright Township, Distr. of Algoma.

Province of Ontario, Department of Mines (1955): Aeromagnetic map, Gladstone Township, Distr. of Algoma.

Province of Ontario, Department of Mines (1955): Aeromagnetic map, Mack Township, Distr. of Algoma. 


